\title{
Deeply virtual Compton scattering off nuclei
}

\author{
A. Kirchner ${ }^{a}$, D. Müller ${ }^{b}$ \\ ${ }^{a}$ Institut für Theoretische Physik, Universität Regensburg \\ D-93040 Regensburg, Germany \\ ${ }^{b}$ Fachbereich Physik, Universität Wuppertal \\ D-42097 Wuppertal, Germany
}

\begin{abstract}
We consider the hard leptoproduction of a photon off nuclei up to spin-1. As a new result we present here the general azimuthal angular dependence of the differential cross section for a spin-1 target. Its twist-two Fourier coefficients of the interference and squared deeply virtual Compton scattering amplitude are evaluated in leading order approximation of perturbation theory in terms of generalized parton distributions, while the pure Bethe-Heitler cross section is exactly calculated in terms of electromagnetic form factors. Relying on a simple model for the nucleon generalized parton distribution $H$, which describes the existing DVCS data for a proton target, we estimate the size of unpolarized cross sections, beam and longitudinal target spin as well as unpolarized charge asymmetries for present fixed target experiments with nuclei. These estimates are confronted with preliminary HERMES data for deuterium and neon.
\end{abstract}

Keywords: deeply virtual Compton scattering, nucleus, deuteron, asymmetries, generalized parton distribution

PACS numbers: 11.80.Cr, 12.38.Bx, 13.60.Fz, 24.85.+p 


\section{Contents}

$\begin{array}{llr}1 & \text { Introduction } & 1\end{array}$

2 Azimuthal angular dependence of the cross section 3

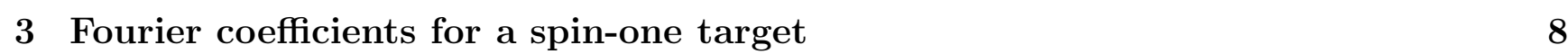

4 Models for nucleus GPDs 12

4.1 Scalar target . . . . . . . . . . . . . . . . . . . . . . . . 12

4.2 GPDs of spin-1/2 nucleus target . . . . . . . . . . . . . . . . . . . 20

4.3 GPDs of spin-one target . . . . . . . . . . . . . . . . . . . . . . . . . . . 21

5 Estimates for observables $\quad 24$

5.1 Analvtical approximation in the valence quark region . . . . . . . . . . . . . . . . 26

5.2 Numerical results . . . . . . . . . . . . . . . . . . . . . . . . . . . . . . . 34

$\begin{array}{llr}6 & \text { Summary } & 39\end{array}$

A Parameterization of the electromagnetic deuteron form factors 41

B Results for the Fourier coefficients 42

B.1 Interference term . . . . . . . . . . . . . . . . . . . . . . . . . 42

B.2 DVCS amplitude squared . . . . . . . . . . . . . . . . . . . . . . . . . . 43

B.3 BH amplitude squared . . . . . . . . . . . . . . . . . . . . . . . . 45

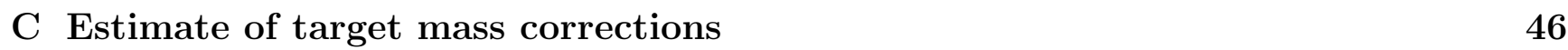

\section{Introduction}

Exclusive two-photon processes in the light-cone dominated region, i.e., in the generalized Bjorken limit, are most suitable for the exploration of the partonic content of hadrons. This comes from the fact that the dominant contribution to the amplitude of such processes arises from Feynman diagrams in which both photons directly couple to one quark line 1]. The processes of interest are different photon-to-meson transition form factors, i.e., $\gamma^{*} \gamma^{(*)} \rightarrow M$, the production of hadron pairs by photon fusion and the crossed processes like $\gamma^{*} H \rightarrow B \gamma$ or $\gamma^{(*)} H \rightarrow B l^{+} l^{-}$. The latter class might be denoted as deeply virtual Compton scattering (DVCS). It contains different processes: DVCS on a hadron target without and with excitation of the final state due to the leptoproduction of a real photon [2, 3, 4, 5, 6, 7], the photoproduction [8] or leptoproduction [9, 10] of a lepton pair.

The factorization of short- and long-range dynamics is formally given by the operator product expansion (OPE) of the time ordered product of two electromagnetic currents, which has been worked out at leading twist-two in next-to-leading order (NLO) and at twist-three level in leading order (LO) of perturbation theory (for references see [7]). However, one should be aware that the partonic hard-scattering part, i.e., the Wilson coefficients, contains collinear singularities, which 
are absorbed in the non-perturbative distributions by a factorization procedure, which has been proven at twist-two level [11, 12].

The non-perturbative distributions are defined in terms of light-ray operators with definite twist sandwiched between the corresponding hadronic states. These distributions, depending on the two-photon process under consideration, are sensitive to different aspects of hadronic physics. Especially, in DVCS one can access the so-called generalized parton distributions (GPDs). The second moment of the flavor singlet GPD is related to the expectation value of the energy momentum tensor. Thus, it contains information on the angular orbital momentum fraction of the nucleon spin carried by quarks [13. Moreover, in contrast to ordinary parton distributions, measurable in inclusive reactions, GPDs carry also information about the parton distribution in transverse direction and might so provide us with a hologramatic picture of the nucleon [14, 15, 16. 17.

Although GPDs are accessible in the leptoproduction of mesons [18, DVCS is the theoretically cleanest tool to probe the partonic content of the nucleon on the level of amplitudes. The DVCS process in the leptoproduction of a photon on a proton target has been measured by the $\mathrm{H} 1$ collaboration [19] in the small $x_{\mathrm{B}}$ region (see also 20]), due to the single beam spin asymmetry by the HERMES [21] and CLAS 22] collaborations as well as due to the charge asymmetry at HERMES [23. Unfortunately, in this DVCS process a deconvolution of GPDs from a measured amplitude is practically impossible. Thus, a model for GPDs is required that satisfies the constraints coming from first principles [1, 2, 3], namely, support properties, sum rules and the reduction to the parton densities in the forward kinematics. Moreover, different authors derived positivity constraints (see Ref. 24] and references therein). Although these derivations are intuitively understandable, a number of questions can be raised that are hardly to answer. At LO in perturbation theory all experimental data are consistent with an oversimplified GPD model [7] that fulfills the first principle and also the positivity constraints, derived in Ref. 25, 26].

Recently, the measurements of beam spin asymmetries in the leptoproduction of a photon on neon and deuteron targets have been reported by the HERMES collaboration [27. Certainly, it is appealing to employ DVCS for the investigation of the internal structure of nuclei. Although the binding energy is negligibly small compared to the virtuality of the exchanged photon, one might expect that DVCS observables are affected by the binding forces. While for spin- 0 and $-1 / 2$ targets one might assume that the nucleus GPDs can be expressed in terms of slightly modified proton GPDs, in the case of a spin-1 target new GPDs enter the DVCS observables, in which bound state effects are dominantly manifested. An appropriate candidate for studying such effects is the deuteron, which has been widely used as a target in lepton-scattering experiments. This nucleus has been extensively studied in both deep-inelastic [28 and elastic [29, 30, 31] scattering. From the theoretical point of view, it would be desirable to have complementary information, which would give us a deeper understanding of the binding forces and could hopefully shed some light of its effective description and the fundamental degree of freedom in QCD.

On the theoretical side it is time to study DVCS on nuclei in more detail. For deuterium a parameterization of leading twist-two operator matrix elements in terms of GPDs has been proposed in Ref. [32. First estimates for the beam spin asymmetry have been presented in Ref. [33, based on a drastic simplification of the theoretical prediction, and in Ref. 34, 35] by means of a convolution model. In Ref. [36 it has been argued that nuclei GPDs can deliver information about the spatial distribution of the strong forces in terms of the fundamental degrees of freedom in QCD. In this paper we complete the twist-two sector for the theoretical predictions for a spin-1 
target, generalize our oversimplified model, and estimate different asymmetries for fixed target experiments.

The outline of the paper is the following: In Section 2 the OPE approach is applied to DVCS on a target with arbitrary spin. Employing these general results, in Section 3 we evaluate the Fourier coefficients appearing in the cross section for a spin-1 target at leading twist and at leading order in perturbation theory. In Section 4 we present oversimplified GPD models for nuclei, expressed by the proton ones, where bound state effects for longitudinal degrees of freedom are neglected. Relying on qualitative properties of proton GPDs, consistent with the DVCS data for the proton target, we estimate in Section 5 by a rough kinematical approximation beam spin, charge, and target spin asymmetries for fixed target experiments at HERMES and JLAB kinematics. We then provide numerical results for these asymmetries and the unpolarized cross section. Finally, we summarize and give conclusions. Appendices are devoted to the parameterization of the deuteron electromagnetic form factors, the results for the Fourier coefficients of a spin-1 target, and the target mass corrections.

\section{Azimuthal angular dependence of the cross section}

In this Section we evaluate the differential cross section for the leptoproduction of a photon

$$
l^{ \pm}(k) \mathrm{A}\left(P_{1}\right) \rightarrow l^{ \pm}\left(k^{\prime}\right) \mathrm{A}\left(P_{2}\right) \gamma\left(q_{2}\right)
$$

off a nucleus target $\mathrm{A}$ with atomic mass number $A$ and mass $M_{\mathrm{A}}$. The goal is to express the azimuthal angular harmonics in terms of the electromagnetic current and Compton amplitudes to twist-two accuracy at LO in $\alpha_{s}$. The considerations are valid for any target with arbitrary spin content.

The five-fold differential cross section

$$
\frac{d \sigma}{d x_{\mathrm{A}} d y d\left|\Delta^{2}\right| d \phi d \varphi}=\frac{\alpha^{3} x_{\mathrm{A}} y}{16 \pi^{2} \mathcal{Q}^{2} \sqrt{1+\epsilon^{2}}}\left|\frac{\mathcal{T}}{e^{3}}\right|^{2}, \quad \epsilon \equiv 2 x_{\mathrm{A}} \frac{M_{\mathrm{A}}}{\mathcal{Q}}
$$

depends on the scaling variable $x_{\mathrm{A}}=\mathcal{Q}^{2} / 2 P_{1} \cdot q_{1}$, where $\mathcal{Q}^{2}=-q_{1}^{2}$ with $q_{1}=k-k^{\prime}$, the momentum transfer square $\Delta^{2}=\left(P_{2}-P_{1}\right)^{2}$, the photon energy fraction $y=P_{1} \cdot q_{1} / P_{1} \cdot k$ and, in general, two azimuthal angles. In the following we refer to the rest frame, shown in Fig. 11 The scaling variable $x_{\mathrm{A}}$ is related to the Bjorken variable $x_{\mathrm{B}}$ by

$$
x_{\mathrm{B}}=\frac{\mathcal{Q}^{2}}{2 M_{N} E y} \approx A x_{\mathrm{A}},
$$

where $M_{N}$ is the nucleon mass and $E$ is the lepton beam energy. The photon energy fraction $y$ and the Bjorken variable satisfy the well know relation

$$
x_{\mathrm{B}} y=\frac{\mathcal{Q}^{2}}{s_{N}-M_{N}^{2}},
$$

where $s_{N}$ is the center-of-mass energy squared for the lepton scattering off a nucleon. Below it is sometimes more convenient to use the scaling variable

$$
\xi \approx-\eta \approx \frac{x_{\mathrm{A}}}{2-x_{\mathrm{A}}} \approx \frac{x_{\mathrm{B}}}{2 A-x_{\mathrm{B}}}
$$




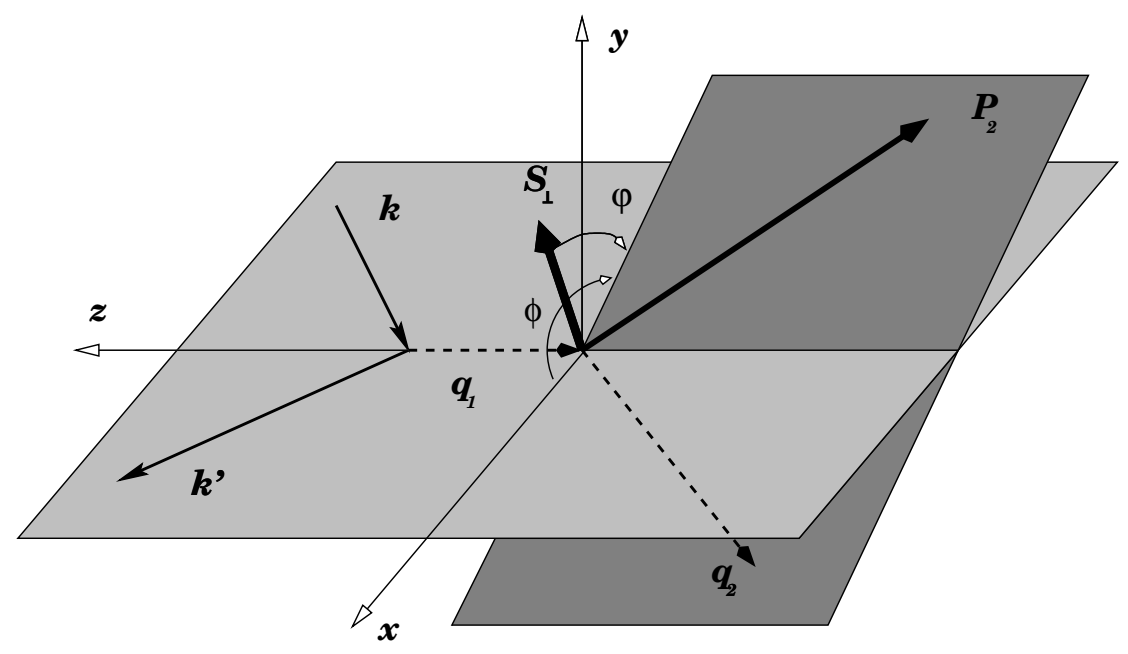

Figure 1: The kinematics of the leptoproduction in the target rest frame. The $z$-direction is chosen counter-along the three-momentum of the incoming virtual photon. The lepton threemomenta form the lepton scattering plane, while the recoiled nucleus and outgoing real photon define the nucleus scattering plane. In this reference system the azimuthal angle of the scattered lepton is $\phi_{l}=0$, while the azimuthal angle between the lepton plane and the recoiled nucleus momentum is $\phi_{N}=\phi$. When the nucleus is transversely polarized (in this reference frame) $S_{\perp}=(0, \cos \Phi, \sin \Phi, 0)$, the angle between the spin vector and the scattered nucleus is denoted as $\varphi=\Phi-\phi_{N}$.

instead of $x_{\mathrm{A}}$, where $\Delta^{2} / \mathcal{Q}^{2}$ corrections have been neglected. Here $\eta$ is called the skewedness parameter, giving the longitudinal momentum fraction in the $t$-channel. As we see, in the DVCS kinematics it is simply related to the Bjorken like scaling variable $\xi$. To define the azimuthal angles we point the virtual photon three-momentum towards the negative $z$-direction. $\phi=\phi_{l}-\phi_{N}$ is the angle between the lepton and nucleus scattering planes and $\varphi=\Phi-\phi_{N}$ is the difference of the azimuthal angle $\Phi$ of the spin vector

$$
S^{\mu}=(0, \cos \Phi \sin \Theta, \sin \Phi \sin \Theta, \cos \Theta),
$$

giving the magnetic quantization direction for the initial nucleus, and the azimuthal angle $\phi_{N}$ of the recoiled nucleus as depicted in Fig. 1

We consider this process in the (generalized) Bjorken limit, i.e., $\mathcal{Q}^{2} \sim P_{1} \cdot q_{1}$ should be large compared to $M_{\mathrm{A}}^{2}$ and $\Delta^{2}$. Obviously, increasing the atomic mass number $A$ one will violate the condition $\mathcal{Q}^{2} \gg M_{\mathrm{A}}^{2}$, since $\mathcal{Q}^{2}$ is in reality restricted by the experimental settings. It is instructive to consider the situation in deeply inelastic scattering. Here the hadronic tensor is given by the absorptive part of the forward scattering amplitude. In the kinematical forward case the target mass corrections are given by $\epsilon^{2}$, which is independent on the mass number $A$. In our kinematics the situation is more complex [37]. Fortunately, it is shown in Appendix C that within our GPD model, specified below, in the DVCS kinematics the target mass corrections ${ }^{1}$ possess the same

\footnotetext{
${ }^{1}$ We distinguish between power suppressed corrections that arise from multi-parton correlation GPDs and target mass corrections. The former ones contain new dynamical information and not much is known about them. The latter ones arise from trace subtraction of twist-two operators. They have been elaborated in Ref. 37] and are given as convolution with so-called double distributions or alternatively with GPDs.
} 


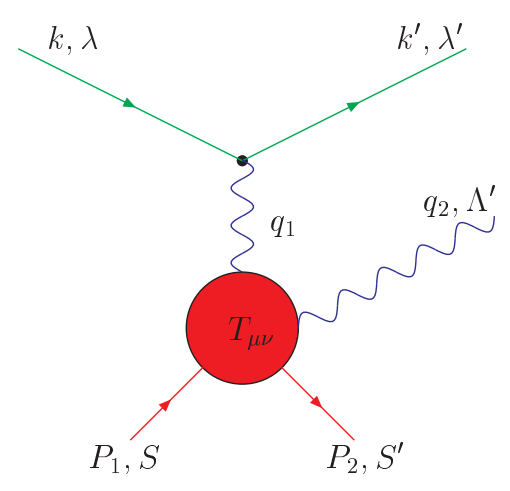

(a)

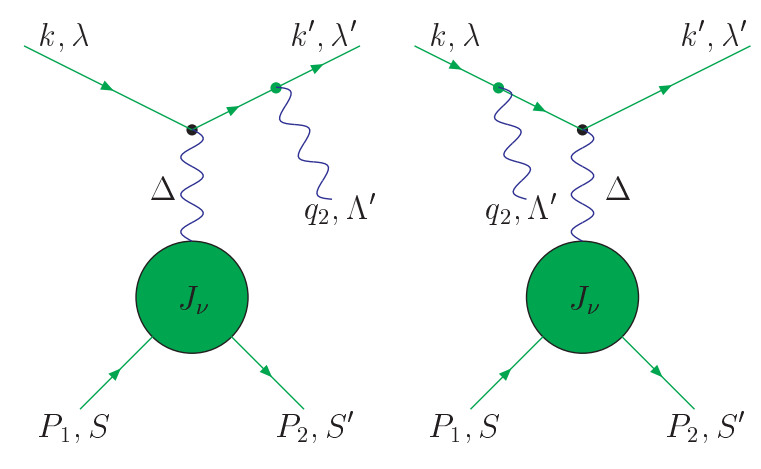

(b)

Figure 2: Both DVCS (a) and BH (b) amplitudes contribute to the hard leptoproduction amplitude of a photon.

$A$ scaling as the twist-two contributions. Thus, they have nearly the same size as for a nucleon target and we may employ the OPE approach also for realistic $\mathcal{Q}^{2}$ and larger $A$.

In the one virtual photon exchange approximation the amplitude $\mathcal{T}$ is the sum of the DVCS $\mathcal{T}_{\text {DVCS }}$ and Bethe-Heitler $(\mathrm{BH}) \mathcal{T}_{\mathrm{BH}}$ ones, displayed in Fig. [2]

$$
\mathcal{T}^{2}=\left\{\left|\mathcal{T}_{\mathrm{BH}}\right|^{2}+\left|\mathcal{T}_{\mathrm{DVCS}}\right|^{2}+\mathcal{I}\right\}
$$

with the interference term

$$
\mathcal{I}=\mathcal{T}_{\mathrm{DVCS}} \mathcal{T}_{\mathrm{BH}}^{*}+\mathcal{T}_{\mathrm{DVCS}}^{*} \mathcal{T}_{\mathrm{BH}}
$$

where the recoiled lepton $\left(\lambda^{\prime}\right)$, nucleus $\left(S^{\prime}\right)$ as well as photon $\left(\Lambda^{\prime}\right)$ polarization will usually not be observed. Each of these three terms in Eq. (77) is given by the contraction of DVCS tensor

$$
T_{\mu \nu}\left(P, \Delta, q \mid S, S^{\prime}\right)=\frac{i}{e^{2}} \int d x \mathrm{e}^{i x \cdot q}\left\langle P_{2}, S^{\prime}\left|T j_{\mu}(x / 2) j_{\nu}(-x / 2)\right| P_{1}, S\right\rangle
$$

or/and electromagnetic current

$$
J_{\alpha}\left(P, \Delta \mid S, S^{\prime}\right)=\frac{1}{e}\left\langle P_{2}, S^{\prime}\left|j_{\alpha}(0)\right| P_{1}, S\right\rangle, \quad j_{\alpha}=\sum_{i=u, d, s} e_{i} \bar{\psi} \gamma_{\alpha} \psi,
$$

with a corresponding leptonic tensor

$$
\begin{array}{rlr}
\left|\mathcal{T}_{\mathrm{DVCS}}\right|^{2} & =\frac{e^{6}}{q_{1}^{4}}\left(-g^{\alpha \beta}\right) L_{\mathrm{DVCS}}^{\mu \nu} \sum_{S^{\prime}} T_{\alpha \mu}\left(T_{\beta \nu}\right)^{\dagger}, \\
\left|\mathcal{T}_{\mathrm{BH}}\right|^{2} & =\frac{e^{6}}{\Delta^{4}} L_{\mathrm{BH}}^{\mu \nu} \sum_{S^{\prime}} J_{\mu} J_{\nu}^{\dagger}, \\
\mathcal{I} & =\frac{ \pm e^{6}}{q_{1}^{2} \Delta^{2}} L^{\alpha \mu \nu} \sum_{S^{\prime}}\left[J_{\mu}\left(T_{\alpha \nu}\right)^{\dagger}+\text { h.c. }\right] \quad\left\{\begin{array}{l}
+ \text { for } e^{-} \\
- \text {for } e^{+}
\end{array}\right.
\end{array}
$$

Here $P=P_{1}+P_{2}$ and $q=\left(q_{1}+q_{2}\right) / 2$ and we summed over the photon $\left(\Lambda^{\prime}\right)$ and lepton $\left(\lambda^{\prime}\right)$ polarizations, where the second summation is included in the leptonic tensors. For any nucleus (or hadron) target a general parameterization of the DVCS tensor at twist-three level and in LO 
approximation of perturbation theory has been given in Ref. 38. To NLO accuracy a new Lorentz structure occurs due to the flip of the photon helicity by two units, which is caused by the so-called twist-two gluonic transversity [39, 40, 41, describing an angular orbital momentum flip by two units, as well as twist-four effects [42. In the following we only consider its twist-two part, where it is expected that for the typical kinematics of present fixed target experiments the twist-three contributions are small for different observables, but not for all of them. In this approximation the DVCS tensor is parameterized by a vector $V_{1}^{\mu}$ and an axial-vector $A_{1}^{\mu}$ part [5]

$$
T_{\mu \nu}=-\mathcal{P}_{\mu}{ }^{\sigma} g_{\sigma \tau} \mathcal{P}^{\tau}{ }_{\nu} \frac{q \cdot V_{1}}{P \cdot q}-\mathcal{P}_{\mu}{ }^{\sigma} i \epsilon_{\sigma \tau q \rho} \mathcal{P}^{\tau}{ }_{\nu} \frac{A_{1}^{\rho}}{P \cdot q},
$$

where the projector $\mathcal{P}_{\mu \nu}=g_{\mu \nu}-q_{1 \mu} q_{2 \nu} / q_{1} \cdot q_{2}$ ensures current conservation. The leptonic tensors are evaluated by means of the standard projector technique and read after summation over the helicity of the final state lepton (see Fig. 2)

$$
\begin{aligned}
L_{\mathrm{DVCS}}^{\mu \nu} & =\sum_{\lambda^{\prime}} \bar{u}\left(k^{\prime}, \lambda^{\prime}\right) \gamma^{\mu} u(k, \lambda) \bar{u}(k, \lambda) \gamma^{\nu} u\left(k^{\prime}, \lambda^{\prime}\right)=\operatorname{Tr} \gamma^{\mu} \not k \frac{1-\lambda \gamma_{5}}{2} \gamma^{\nu} \not k^{\prime} \\
& =2\left(k^{\mu} k^{\prime \nu}+k^{\nu} k^{\prime \mu}-k \cdot k^{\prime} g^{\mu \nu}-i \lambda \epsilon^{\mu \nu \alpha \beta} k_{\alpha} k_{\beta}^{\prime}\right) \\
L_{\mathrm{BH}}^{\mu \nu} & =\left(-g_{\alpha \beta}\right) \operatorname{Tr} \Gamma^{\alpha \mu}\left(k, k^{\prime}, \Delta\right) \not k \frac{1-\lambda \gamma_{5}}{2} \Gamma^{\nu \beta}\left(k, k^{\prime}, \Delta\right) k^{\prime} \\
L^{\alpha \mu \nu} & =\left(-g_{\beta}^{\alpha}\right) \operatorname{Tr} \Gamma^{\beta \mu}\left(k, k^{\prime}, \Delta\right) \not k \frac{1-\lambda \gamma_{5}}{2} \gamma^{\nu} \not k^{\prime}
\end{aligned}
$$

where

$$
\Gamma_{\alpha \mu}\left(k, k^{\prime}, \Delta\right)=\frac{\gamma_{\alpha}(\not k-\not \Delta) \gamma_{\mu}}{(k-\Delta)^{2}}+\frac{\gamma_{\mu}\left(\not k^{\prime}+\not \Delta\right) \gamma_{\alpha}}{\left(k^{\prime}+\Delta\right)^{2}}
$$

is the leptonic part of the $\mathrm{BH}$ amplitude.

Employing the parameterizations (31), (33) and (34), the contractions of leptonic and DVCS tensors result in the kinematically exact expression for the squared $\mathrm{BH}$ term (of course, in tree approximation)

$$
\begin{aligned}
\left|\mathcal{T}_{\mathrm{BH}}\right|^{2}=\frac{-8 e^{6}}{\mathcal{P}_{1}(\phi) \mathcal{P}_{2}(\phi) \mathcal{Q}^{4} \Delta^{2}} \sum_{S^{\prime}} & \left\{q \cdot J k \cdot J^{\dagger}+k \cdot J q \cdot J^{\dagger}-q \cdot J q \cdot J^{\dagger}-2 k \cdot J k \cdot J^{\dagger}\right. \\
& -\frac{\left(\mathcal{Q}^{2}-\Delta^{2}+2 k \cdot \Delta\right)^{2}+4(k \cdot \Delta)^{2}}{4 \Delta^{2}} J \cdot J^{\dagger} \\
& \left.+\frac{i \lambda\left[\left(\mathcal{Q}^{2}+4 k \cdot \Delta-\Delta^{2}\right) \epsilon_{q \Delta J J^{\dagger}}+\Delta^{2} \epsilon_{k(2 q+\Delta) J J^{\dagger}}\right]}{2 \Delta^{2}}\right\},
\end{aligned}
$$

while the interference term ${ }^{2}$ (here for negatively charged lepton scattering)

$$
\begin{aligned}
\mathcal{I}= & \frac{2-2 y+y^{2}}{y^{2} \mathcal{P}_{1}(\phi) \mathcal{P}_{2}(\phi)} \frac{4 e^{6} \xi}{\Delta^{2} \mathcal{Q}^{4}}\left(k^{\sigma}-\frac{q^{\sigma}}{y}\right) \sum_{S^{\prime}}\left[\left(J_{\sigma}+2 \Delta_{\sigma} \frac{q \cdot J}{\mathcal{Q}^{2}}\right) q \cdot V_{1}^{\dagger}+2 i \epsilon_{\sigma q \Delta J} \frac{q \cdot A_{1}^{\dagger}}{\mathcal{Q}^{2}}\right] \\
& +\frac{\lambda(2-y) y}{y^{2} \mathcal{P}_{1}(\phi) \mathcal{P}_{2}(\phi)} \frac{4 e^{6} \xi}{\Delta^{2} \mathcal{Q}^{4}}\left(k^{\sigma}-\frac{q^{\sigma}}{y}\right) \sum_{S^{\prime}}\left[\left(J_{\sigma}+2 \Delta_{\sigma} \frac{q \cdot J}{\mathcal{Q}^{2}}\right) q \cdot A_{1}^{\dagger}+2 i \epsilon_{\sigma q \Delta J} \frac{q \cdot V_{1}^{\dagger}}{\mathcal{Q}^{2}}\right]+\text { h.c. }
\end{aligned}
$$

\footnotetext{
${ }^{2}$ Note that the sign in front of the Levi-Civita tensors in Eqs. (20) and (23) of Ref. [5] is erroneously.
} 
and the squared DVCS amplitude

$$
\begin{aligned}
\left|\mathcal{T}_{\mathrm{DVCS}}\right|^{2}= & 8 e^{6} \frac{2-2 y+y^{2}}{y^{2}} \frac{\xi^{2}}{\mathcal{Q}^{6}} \sum_{S^{\prime}}\left(q \cdot V_{1} q \cdot V_{1}^{\dagger}+q \cdot A_{1} q \cdot A_{1}^{\dagger}\right) \\
& +8 e^{6} \frac{\lambda(2-y)}{y} \frac{\xi^{2}}{\mathcal{Q}^{6}} \sum_{S^{\prime}}\left(q \cdot V_{1} q \cdot A_{1}^{\dagger}+q \cdot A_{1} q \cdot V_{1}^{\dagger}\right)
\end{aligned}
$$

are expanded with respect to $1 / \mathcal{Q}$. In contrast to the squared DVCS amplitude the interference as well as the squared $\mathrm{BH}$ terms have an additional $\phi$-dependence due to the (scaled) $\mathrm{BH}$ propagators

$$
\begin{aligned}
& \mathcal{P}_{1} \equiv \frac{\left(k-q_{2}\right)^{2}}{\mathcal{Q}^{2}}=-\frac{1}{y\left(1+\epsilon^{2}\right)}\{J+2 K \cos (\phi)\} \\
& \mathcal{P}_{2} \equiv \frac{(k-\Delta)^{2}}{\mathcal{Q}^{2}}=1+\frac{\Delta^{2}}{\mathcal{Q}^{2}}+\frac{1}{y\left(1+\epsilon^{2}\right)}\{J+2 K \cos (\phi)\}
\end{aligned}
$$

where

$$
J=\left(1-y-\frac{y \epsilon^{2}}{2}\right)\left(1+\frac{\Delta^{2}}{\mathcal{Q}^{2}}\right)-(1-x)(2-y) \frac{\Delta^{2}}{\mathcal{Q}^{2}}
$$

and

$$
K^{2}=-\frac{\Delta^{2}}{\mathcal{Q}^{2}}\left(1-x_{\mathrm{A}}\right)\left(1-y-\frac{y^{2} \epsilon^{2}}{4}\right)\left(1-\frac{\Delta_{\min }^{2}}{\Delta^{2}}\right)\left\{\sqrt{1+\epsilon^{2}}+\frac{4 x_{\mathrm{A}}\left(1-x_{\mathrm{A}}\right)+\epsilon^{2}}{4\left(1-x_{\mathrm{A}}\right)} \frac{\Delta^{2}-\Delta_{\min }^{2}}{\mathcal{Q}^{2}}\right\}
$$

with the plus sign taken for the square root in Eq. (22). It vanishes at the kinematical boundary $\Delta^{2}=\Delta_{\text {min }}^{2}$, determined by the minimal value

$$
-\Delta_{\min }^{2}=\mathcal{Q}^{2} \frac{2\left(1-x_{\mathrm{A}}\right)\left(1-\sqrt{1+\epsilon^{2}}\right)+\epsilon^{2}}{4 x_{\mathrm{A}}\left(1-x_{\mathrm{A}}\right)+\epsilon^{2}} \approx \frac{M_{\mathrm{A}}^{2} x_{\mathrm{A}}^{2}}{1-x_{\mathrm{A}}+x_{\mathrm{A}} M_{\mathrm{A}}^{2} / \mathcal{Q}^{2}},
$$

as well as at

$$
y\left(x, \mathcal{Q}^{2}\right)=y_{\max } \equiv 2 \frac{\sqrt{1+\epsilon^{2}}-1}{\epsilon^{2}} \approx 1-\frac{M_{\mathrm{A}}^{2} x_{\mathrm{A}}^{2}}{\mathcal{Q}^{2}} .
$$

In the frame chosen the contractions of leptonic, depending on $k, q_{1}$, and $\Delta$, and DVCS, a function of $q, P, \Delta$ and the spin vector $S$, tensors in Eqs. (15) 17), yield finite sums of Fourier harmonics [4, 7]:

$$
\begin{aligned}
& \left|\mathcal{T}_{\mathrm{BH}}\right|^{2}=\frac{e^{6}\left(1+\epsilon^{2}\right)^{-2}}{x_{\mathrm{A}}^{2} y^{2} \Delta^{2} \mathcal{P}_{1}(\phi) \mathcal{P}_{2}(\phi)}\left\{c_{0}^{\mathrm{BH}}+\sum_{n=1}^{2}\left[c_{n}^{\mathrm{BH}} \cos (n \phi)+s_{n}^{\mathrm{BH}} \sin (\phi)\right]\right\}, \\
& \left|\mathcal{T}_{\mathrm{DVCS}}\right|^{2}=\frac{e^{6}}{y^{2} \mathcal{Q}^{2}}\left\{c_{0}^{\mathrm{DVCS}}+\sum_{n=1}^{2}\left[c_{n}^{\mathrm{DVCS}} \cos (n \phi)+s_{n}^{\mathrm{DVCS}} \sin (n \phi)\right]\right\}, \\
& \mathcal{I}=\frac{ \pm e^{6}}{x_{\mathrm{A}} y^{3} \Delta^{2} \mathcal{P}_{1}(\phi) \mathcal{P}_{2}(\phi)}\left\{c_{0}^{\mathcal{I}}+\sum_{n=1}^{3}\left[c_{n}^{\mathcal{I}} \cos (n \phi)+s_{n}^{\mathcal{I}} \sin (n \phi)\right]\right\}
\end{aligned}
$$


where the $+(-)$ sign in the interference term stands for the negatively (positively) charged lepton beam. In the twist-two sector the $\phi$ independent contribution of the squared DVCS amplitude is

$$
\begin{aligned}
c_{0}^{\mathrm{DVCS}}=8 \xi^{2} \sum_{S^{\prime}} & {\left[\left(2-2 y+y^{2}\right) \frac{q \cdot V_{1} q \cdot V_{1}^{\dagger}+q \cdot A_{1} q \cdot A_{1}^{\dagger}}{\mathcal{Q}^{4}}\right.} \\
& \left.+\lambda(2-y) y \frac{q \cdot V_{1} q \cdot A_{1}^{\dagger}+q \cdot A_{1} q \cdot V_{1}^{\dagger}}{\mathcal{Q}^{4}}\right],
\end{aligned}
$$

while the Fourier coefficients of the interference term are

$$
\begin{aligned}
\left\{\begin{array}{c}
c_{1}^{\mathcal{I}} \\
s_{1}^{\mathcal{I}}
\end{array}\right\}=8 & \xi x_{\mathrm{A}}\left\{\begin{array}{c}
\Re \mathrm{e} \\
\Im \mathrm{m}
\end{array}\right\} \frac{y k^{\sigma}-q^{\sigma}}{\mathcal{Q}^{2}} \\
\times & \times \sum_{S^{\prime}}\left\{\left(2-2 y+y^{2}\right)\left[\left(J_{\sigma}+2 \Delta_{\sigma} \frac{q \cdot J}{\mathcal{Q}^{2}}\right) \frac{q \cdot V_{1}^{\dagger}}{\mathcal{Q}^{2}}+\frac{2 i \epsilon_{\sigma q \Delta J}}{\mathcal{Q}^{2}} \frac{q \cdot A_{1}^{\dagger}}{\mathcal{Q}^{2}}\right]\right. \\
& \left.+\lambda(2-y) y\left[\left(J_{\sigma}+2 \Delta_{\sigma} \frac{q \cdot J}{\mathcal{Q}^{2}}\right) \frac{q \cdot A_{1}^{\dagger}}{\mathcal{Q}^{2}}+\frac{2 i \epsilon_{\sigma q \Delta J}}{\mathcal{Q}^{2}} \frac{q \cdot V_{1}^{\dagger}}{\mathcal{Q}^{2}}\right]\right\},
\end{aligned}
$$

expanded in leading order of $1 / \mathcal{Q}$. Note that the $\cos (3 \phi)$ and $\cos (2 \phi)$ harmonics in the interference and squared DVCS term, respectively, also appear at twist-two level. Since they arise from the gluon transversity, they are suppressed by $\alpha_{s} / \pi$.

\section{Fourier coefficients for a spin-one target}

So far the formalism, presented in the previous section, is rather general and at LO of perturbation theory it can be easily extended to the twist-three sector. Introducing an appropriate form factor decomposition of the electromagnetic current and the DVCS amplitudes, it can be employed for any target. The resulting Fourier coefficients for the spin-0 and $-1 / 2$ targets are presented at twistthree level in Refs. [6, 7] for a pion ${ }^{3}$ and proton target, respectively. The results for a nucleus target follow from the replacements of kinematical variables, form factors $F_{i}\left(\Delta^{2}\right)$ and (set) of GPDs:

$$
\begin{aligned}
& x_{\mathrm{B}} \rightarrow x_{\mathrm{A}}, \quad \xi \rightarrow \xi, \quad M \rightarrow M_{A}, \quad F_{i}\left(\Delta^{2}\right) \rightarrow F_{i}^{\mathrm{A}}\left(\Delta^{2}\right), \\
& F=\{H, E, \widetilde{H}, \widetilde{E},\}\left(x, \eta, \Delta^{2}\right) \rightarrow F^{\mathrm{A}}=\left\{H^{\mathrm{A}}, E^{\mathrm{A}}, \widetilde{H}^{\mathrm{A}}, \widetilde{E}^{\mathrm{A}},\right\}\left(x, \eta, \Delta^{2}\right) .
\end{aligned}
$$

Let us now derive the Fourier coefficients for a spin-1 target in terms of GPDs. The electromagnetic current

$$
J_{\mu}=-\epsilon_{2}^{*} \cdot \epsilon_{1} P_{\mu} G_{1}+\left(\epsilon_{2}^{*} \cdot P \epsilon_{1 \mu}+\epsilon_{1} \cdot P \epsilon_{2 \mu}^{*}\right) G_{2}-\epsilon_{2}^{*} \cdot P \epsilon_{1} \cdot P \frac{P_{\mu}}{2 M_{\mathrm{A}}^{2}} G_{3}
$$

is given by three form factors $G_{i}\left(\Delta^{2}\right)$ with $i=\{1,2,3\}$, where $\epsilon_{1 \mu}\left(\epsilon_{2 \mu}\right)$ denote the three polarization vectors for the initial (final) nucleus. The form factors $G_{i}\left(\Delta^{2}\right)$ can be measured due to the

\footnotetext{
${ }^{3}$ Here the variable $x_{\mathrm{B}}$ has be defined in the rest frame with respect to the target mass rather than to the nucleon mass.
} 
elastic scattering of a lepton on a nucleus. For the deuteron their parameterizations are available in the literature, see Ref. [30, 31] and references therein.

At twist-two level the amplitudes $V_{1}$ and $A_{1}$, appearing in the parameterization (14) of the DVCS tensor, can be decomposed in a complete basis of nine Compton form factors (CFFs)

$$
\mathcal{F}\left(\xi, \Delta^{2}, \mathcal{Q}^{2}\right)=\left\{\mathcal{H}_{1}, \mathcal{H}_{2}, \mathcal{H}_{3}, \mathcal{H}_{4}, \mathcal{H}_{5}, \widetilde{\mathcal{H}}_{1}, \widetilde{\mathcal{H}}_{2}, \widetilde{\mathcal{H}}_{3}, \widetilde{\mathcal{H}}_{4}\right\}\left(\xi, \Delta^{2}, \mathcal{Q}^{2}\right)
$$

For convenience we employed here the scaling variable $\xi$, c.f., Eq. (15). Adopting the notation of Ref. [32, the CFFs read for the vector

$$
\begin{aligned}
V_{1 \mu}= & -\epsilon_{2}^{*} \cdot \epsilon_{1} P_{\mu} \mathcal{H}_{1}+\left(\epsilon_{2}^{*} \cdot P \epsilon_{1 \mu}+\epsilon_{1} \cdot P \epsilon_{2 \mu}^{*}\right) \mathcal{H}_{2}-\epsilon_{2}^{*} \cdot P \epsilon_{1} \cdot P \frac{P_{\mu}}{2 M_{\mathrm{A}}^{2}} \mathcal{H}_{3} \\
& +\left(\epsilon_{2}^{*} \cdot P \epsilon_{1 \mu}-\epsilon_{1} \cdot P \epsilon_{2 \mu}^{*}\right) \mathcal{H}_{4}+\left(\frac{2 M_{\mathrm{A}}^{2}\left\{\epsilon_{2}^{*} \cdot q \epsilon_{1 \mu}+\epsilon_{1} \cdot q \epsilon_{2 \mu}^{*}\right\}}{P \cdot q}+\frac{\epsilon_{2}^{*} \cdot \epsilon_{1}}{3} P_{\mu}\right) \mathcal{H}_{5},
\end{aligned}
$$

and axial-vector

$$
\begin{aligned}
A_{1_{\mu}}= & i \epsilon_{\mu \epsilon_{2}^{*} \epsilon_{1} P} \widetilde{\mathcal{H}}_{1}-\frac{i \epsilon_{\mu \Delta P \epsilon_{1}} \epsilon_{2}^{*} \cdot P+i \epsilon_{\mu \Delta P \epsilon_{2}^{*}} \epsilon_{1} \cdot P}{M_{\mathrm{A}}^{2}} \widetilde{\mathcal{H}}_{2} \\
& -\frac{i \epsilon_{\mu \Delta P \epsilon_{1}} \epsilon_{2}^{*} \cdot P-i \epsilon_{\mu \Delta P \epsilon_{2}^{*}} \epsilon_{1} \cdot P}{M_{\mathrm{A}}^{2}} \widetilde{\mathcal{H}}_{3}-\frac{i \epsilon_{\mu \Delta P \epsilon_{1}} \epsilon_{2}^{*} \cdot q+i \epsilon_{\mu \Delta P \epsilon_{2}^{*}} \epsilon_{1} \cdot q}{q \cdot P} \widetilde{\mathcal{H}}_{4},
\end{aligned}
$$

where $1 / \mathcal{Q}$-power suppressed effects have been neglected.

The CFFs in Eqs. (33) and (34) are given by a convolution of perturbatively calculable coefficient functions $C^{( \pm)}$and twist-two GPDs via

$$
\begin{aligned}
& \mathcal{H}_{k}=\sum_{i=u, \ldots} \int_{-1}^{1} d x C_{i}^{(-)}\left(\xi, x, \mathcal{Q}^{2}, \mu^{2}\right) H_{k}^{i}\left(x, \eta, \Delta^{2}, \mu^{2}\right)_{\mid \eta=-\xi}, \quad \text { for } \quad k=\{1, \ldots, 5\}, \\
& \widetilde{\mathcal{H}}_{k}=\sum_{i=u, \ldots} \int_{-1}^{1} d x C_{i}^{(+)}\left(\xi, x, \mathcal{Q}^{2}, \mu^{2}\right) \widetilde{H}_{k}^{i}\left(x, \eta, \Delta^{2}, \mu^{2}\right)_{\mid \eta=-\xi}, \quad \text { for } \quad k=\{1, \ldots, 4\},
\end{aligned}
$$

where $\mu$ denotes the factorization scale. For each quark species $i$ we have nine GPDs. The two sets $\left\{H_{1}^{i}, \ldots, H_{5}^{i}\right\}$ and $\left\{\widetilde{H}_{1}^{i}, \ldots, \widetilde{H}_{4}^{i}\right\}$ are defined by off-forward matrix elements of vector and axialvector light-ray operators. All GPDs of a given set satisfy the same evolution equations, which govern the logarithmical dependence on the factorization scale $\mu$. The coefficient functions $C^{(\mp)}$ have been perturbatively expanded. In LO they read for the even $(-)$ and odd $(+)$ parity sectors

$$
\xi C_{(0) i}^{(\mp)}(\xi, x)=\frac{Q_{i}^{2}}{1-x / \xi-i 0} \mp \frac{Q_{i}^{2}}{1+x / \xi-i 0},
$$

where $Q_{i}$ is the fractional quark charge.

The Fourier coefficients $c / s_{i}$ can be calculated from Eqs. (20), (21), and analogous ones for the squared $\mathrm{BH}$ amplitude by summing over the polarization $\Lambda^{\prime}$, where we can employ the common projector technique. For a massive spin-1 particle we have (see for instance [43])

$$
\begin{aligned}
\epsilon_{1 \mu}^{*}(\Lambda=0) \epsilon_{1 \nu}(\Lambda=0) & =S_{\mu} S_{\nu} \\
\epsilon_{1 \mu}^{*}(\Lambda= \pm 1) \epsilon_{1 \nu}(\Lambda= \pm 1) & =\frac{1}{2}\left(-g_{\mu \nu}+\frac{P_{1 \mu} P_{1 \nu}}{M_{\mathrm{A}}^{2}}-S_{\mu} S_{\nu}+\frac{i \Lambda}{M_{\mathrm{A}}} \epsilon_{\mu \nu P_{1} S}\right)
\end{aligned}
$$


where $\Lambda=\{+1,0,-1\}$ denotes the magnetic quantum number with respect to the quantization direction given by the spin vector $S_{\mu}$, defined in Eq. (6). Obviously, the spin sum of the recoiled nucleus is

$$
\sum_{\Lambda^{\prime}=-1}^{1} \epsilon_{2 \mu}^{*}\left(\Lambda^{\prime}\right) \epsilon_{2 \nu}\left(\Lambda^{\prime}\right)=-g_{\mu \nu}+\frac{P_{2 \mu} P_{2 \nu}}{M_{\mathrm{A}}^{2}}
$$

As we see, the Fourier coefficients for a spin-1 target quadratically depend on the spin vector $S_{\mu}$ and, thus, we introduce the following decomposition

$$
\begin{aligned}
c_{n}^{T}= & \frac{3}{2} \Lambda^{2} c_{n, \mathrm{unp}}^{T}+\Lambda\left\{c_{n, \mathrm{LP}}^{T} \cos (\Theta)+c_{n, \mathrm{TP}}^{T}(\varphi) \sin (\Theta)\right\}+\left(1-\frac{3}{2} \Lambda^{2}\right) \\
& \times\left\{c_{n, \mathrm{LTP}}^{T}(\varphi) \sin (2 \Theta)+c_{n, \mathrm{LLP}}^{T} \cos ^{2}(\Theta)+c_{n, \mathrm{TTP}}^{T}(\varphi) \sin ^{2}(\Theta)\right\}
\end{aligned}
$$

for $T=\{\mathrm{BH}, \mathcal{I}, \mathrm{DVCS}\}$. An analogous decomposition holds true for the odd harmonics $s_{n}^{T}$. The unpolarized and the longitudinally polarized coefficients $c / s_{n, \text { unp }}^{T}, c / s_{n, \mathrm{LP}}^{T}$, and $c / s_{n, \mathrm{LLP}}^{T}$, respectively, are independent of $\varphi$. The transverse coefficients $c / s_{n, \mathrm{TP}}^{T}$ and the transverse-longitudinal interference terms may be decomposed with respect to the first harmonics in the azimuthal angle $\varphi$

$$
\begin{aligned}
c_{n, \mathrm{TP}}^{T}(\varphi) & =c_{n, \mathrm{TP}+}^{T} \cos (\varphi)+s_{n, \mathrm{TP}-}^{T} \sin (\varphi), \\
c_{n, \mathrm{LTP}}^{T}(\varphi) & =c_{n, \mathrm{LTP}+}^{T} \cos (\varphi)+s_{n, \mathrm{LTP}-}^{T} \sin (\varphi),
\end{aligned}
$$

while $c / s_{n, \text { TTP }}^{T}$ may be written as

$$
c_{n, \mathrm{TTP}}^{T}(\varphi)=c_{n, \mathrm{TTP} \Sigma}^{T}+c_{n, \mathrm{TTP} \Delta}^{T} \cos (2 \varphi)+s_{n, \mathrm{TTP} \pm}^{T} \sin (2 \varphi) .
$$

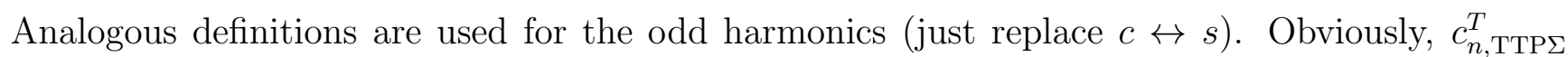
does not belong to an independent frequency, rather it can be included in the constant and $\cos ^{2}(\theta)$ terms of Eq. (40). Indeed, the calculation of the Fourier coefficients establishes the equality:

$$
c_{0, \mathrm{LLP}}^{T}=3 c_{0, \mathrm{unp}}^{T}-2 c_{0, \mathrm{TTP} \Sigma}^{T}, \quad T=\{\mathrm{BH}, \mathcal{I}, \mathrm{DVCS}\},
$$

and an analogous one for $s_{1, \text { TTP }}^{\mathcal{I}}$ coefficients.

We will now present the results for the dominant harmonics $c / s_{1}^{\mathcal{I}}$ of the interference term. We write them as a product of leptonic prefactors $\mathcal{L}$, defined in Tab. 1, with the real/imaginary part of 'universal' functions $\mathcal{C}_{i}^{\mathcal{I}}$ :

$$
\left\{\begin{array}{c}
c_{1, i}^{\mathcal{I}} \\
s_{1, i}^{\mathcal{I}}
\end{array}\right\}=\left\{\begin{array}{c}
\mathcal{L}_{1, i}^{\mathcal{I} c} \\
\mathcal{L}_{1, i}^{\mathcal{I} s}
\end{array}\right\}\left\{\begin{array}{c}
\Re \mathrm{e} \\
\Im \mathrm{m}
\end{array}\right\} \mathcal{C}_{i}^{\mathcal{I}}, \quad \text { for } \quad i=\{\text { unp }, \cdots, \mathrm{TTP}-\}
$$

Since they linearly depend on the nine CFFs and three electromagnetic form factors, we write these functions in terms of real valued $9 \times 3$ matrices $\mathcal{M}_{\mathrm{A}}$ :

$$
\mathcal{C}_{i}^{\mathcal{I}}=\left(\mathcal{H}_{1} \cdots \mathcal{H}_{5} \widetilde{\mathcal{H}}_{1} \cdots \widetilde{\mathcal{H}}_{4}\right) \mathcal{M}_{i}^{\mathcal{I}}\left(\begin{array}{c}
G_{1} \\
G_{2} \\
G_{3}
\end{array}\right), \quad \text { for } \quad i=\{\text { unp, } \cdots, \mathrm{TTP}-\}
$$




\begin{tabular}{|c|c|c|c|}
\hline $\mathrm{i}$ & $\mathcal{L}_{1, i}^{\mathcal{I} c}$ & $\mathcal{L}_{1, i}^{\mathcal{I} s}$ & $\mathcal{L}_{0, i}^{\mathrm{DVCS}}$ \\
\hline \hline $\mathrm{unp}$ & $-8 K\left(2-2 y+y^{2}\right)$ & $8 \lambda K y(2-y)$ & $2-2 y+y^{2}$ \\
\hline $\mathrm{LP}$ & $-8 \lambda K y(2-y)$ & $8 K\left(2-2 y+y^{2}\right)$ & $\lambda y(2-y)$ \\
\hline $\mathrm{TP}+$ & $-8 \lambda \frac{\sqrt{1-y} M_{\mathrm{A}}}{\mathcal{Q}} y(2-y)$ & $8 \frac{\sqrt{1-y} M_{\mathrm{A}}}{\mathcal{Q}}\left(2-2 y+y^{2}\right)$ & $\lambda \frac{\mathcal{Q} K}{\sqrt{1-y} M_{\mathrm{A}}} y(2-y)$ \\
\hline $\mathrm{TP}-$ & $8 \lambda \frac{\sqrt{1-y} M_{\mathrm{A}}}{\mathcal{Q}} y(2-y)$ & $8 \frac{\sqrt{1-y} M_{\mathrm{A}}}{\mathcal{Q}}\left(2-2 y+y^{2}\right)$ & $-\frac{\mathcal{Q} K}{M_{\mathrm{A}} \sqrt{1-y}}\left(2-2 y+y^{2}\right)$ \\
\hline $\mathrm{LLP}$ & $-8 K\left(2-2 y+y^{2}\right)$ & $8 \lambda K y(2-y)$ & $2-2 y+y^{2}$ \\
\hline $\mathrm{LTP}+$ & $-8 \frac{\sqrt{1-y} M_{\mathrm{A}}}{\mathcal{Q}}\left(2-2 y+y^{2}\right)$ & $8 \lambda \frac{\sqrt{1-y} M_{\mathrm{A}}}{\mathcal{Q}} y(2-y)$ & $\frac{\mathcal{Q} K}{M_{\mathrm{A}} \sqrt{1-y}}\left(2-2 y+y^{2}\right)$ \\
\hline $\mathrm{LTP}-$ & $8 \frac{\sqrt{1-y} M_{\mathrm{A}}}{\mathcal{Q}}\left(2-2 y+y^{2}\right)$ & $8 \frac{\sqrt{1-y} M_{\mathrm{A}}}{\mathcal{Q}} \lambda y(2-y)$ & $\lambda \frac{\mathcal{Q} K}{\sqrt{1-y} M_{\mathrm{A}}} y(2-y)$ \\
\hline $\mathrm{TTP} \Sigma$ & $-8 K\left(2-2 y+y^{2}\right)$ & $8 \lambda K y(2-y)$ & $2-2 y+y^{2}$ \\
\hline $\mathrm{TTP} \Delta$ & $-8 K\left(2-2 y+y^{2}\right)$ & $8 \lambda K y(2-y)$ & $2-2 y+y^{2}$ \\
\hline $\mathrm{TTP} \pm$ & $8 K\left(2-2 y+y^{2}\right)$ & $8 \lambda K y(2-y)$ & $-\lambda y(2-y)$ \\
\hline
\end{tabular}

Table 1: Kinematical prefactors that appear in the Fourier coefficients of the interference term (45) and squared DVCS amplitude (47).

In the case of a longitudinally polarized target the three matrices $\mathcal{M}_{\text {unp }}^{\mathcal{I}}, \mathcal{M}_{\text {LP }}^{\mathcal{I}}$, and $\mathcal{M}_{\text {LLP }}^{\mathcal{I}}$ are presented in Appendix B.1 for small values of the momentum transfer ${ }^{4}$, i.e., $-\Delta^{2} \ll M_{\mathrm{A}}^{2}$.

The squared DVCS amplitude is written in an analogous manner, namely,

$$
c_{0, i}^{\mathrm{DVCS}}=\mathcal{L}_{0, i}^{\mathrm{DVCS}} \mathcal{C}_{i}^{\mathrm{DVCS}}, \quad \text { for } i=\{\mathrm{unp}, \cdots, \mathrm{TTP}-\},
$$

where the nine functions

$$
\mathcal{C}_{i}^{\text {DVCS }}=\left(\mathcal{H}_{1} \cdots \widetilde{\mathcal{H}}_{4}\right) \mathcal{M}_{i}^{\text {DVCS }}\left(\begin{array}{c}
\mathcal{H}_{1}^{*} \\
\vdots \\
\widetilde{\mathcal{H}}_{4}^{*}
\end{array}\right)
$$

are expressed by $9 \times 9$ hermitian matrices $\mathcal{M}_{i}^{\text {DVCS }}$. The leptonic factors $\mathcal{L}_{0, i}^{\text {DVCS }}$ are given in Tab. 1] and the approximation of the matrices for longitudinally polarized target can be found in Appendix B.2. The analogous BH contributions are listed in Appendix B.3.

Let us summarize. For a given harmonic in $\phi$ we have altogether nine possible observables. In principle, they can be measured by an appropriate adjustment of the spin vector $S_{\mu}$ and Fourier analysis with respect to the azimuthal angle $\varphi$. The interference term linearly depends on the CFFs and is, thus, of special interest. In facilities that have both kinds of leptons it can be separated by means of the charge asymmetry. Combined with single spin-flip and unpolarized

4 The complete expressions for all matrices of the interference and squared DVCS terms as well as the complete squared $\mathrm{BH}$ term are available as TeX or MATHEMATICA file from the authors via e-mail: axel.kirchner@physik.uni-regensburg.de, dieter.mueller@.physik.uni-regensburg.de (dmueller@theorie.physik.uniwuppertal.de). 
as well as double spin-flip measurements, the sin/cos harmonics can be separated. This would provide access to the imaginary and real part of the nine linear combinations $\mathcal{C}_{i}^{\mathcal{I}}$ and so to the nine GPDs. To extract information from the nine GPDs one needs a realistic model for them, where the model parameters have to be adjusted by fitting the measured data. Certainly, this goal requires a deep understanding of non-perturbative physics and a dedicated facility.

\section{Models for nucleus GPDs}

The main uncertainty in the estimate of observables is the lack of knowledge on GPDs. Since GPDs are hybrids of exclusive and inclusive quantities, we may expect that the constraints on models arising from the reduction to parton densities in the forward limit or to elastic electromagnetic form factors, appearing as the lowest moment of GPDs, ensure the right order of magnitude for cross sections and asymmetries. However, one should keep in mind that the predictions for DVCS in terms of GPDs are rather complex and that only few quantities, like the sign and the size of the single beam spin asymmetry or the size of the unpolarized cross section, are "predictable" in a rather model independent manner. We can also rephrase this statement in a positive sentence, namely, most of the observables are sensitive to details of the GPD models. One example is for instance the charge asymmetry for DVCS on an unpolarized proton target. However, we should emphasize again that the interpretation of experimental data requires a careful consideration of possible kinematical effects as it has been stressed in [7].

\subsection{Scalar target}

To start with the simplest case, we want to consider a scalar nucleus target A with charge $Z e$. In this case only one GPD arises at twist-two level and two of them at twist-three level [38, 6, 44, 45]. The former one is defined as expectation value of twist-two light-ray operators

$$
H^{i / \mathrm{A}}\left(x, \eta, \Delta, \mathcal{Q}^{2}\right)=\left.\int \frac{d \kappa}{2 \pi} e^{i \kappa P_{+} x}\left\langle\mathrm{~A}\left(P_{2}\right)\left|\bar{\psi}_{i}(-\kappa n) \gamma_{+} \psi_{i}(\kappa n)\right| \mathrm{A}\left(P_{1}\right)\right\rangle\right|_{\eta=\frac{\Delta_{+}}{P_{+}}},
$$

where the gauge link has been omitted. Here the +- component of a four vector is given by the contraction with the light-like vector $n$, e.g., $P_{+}=n \cdot P$. Thus, $-1 \leq x \leq 1$ can be interpreted as a momentum fraction with respect to the light-cone component $P_{+}$, while the skewedness parameter $\eta$ appears as a scaling variable. Lorentz invariance requires that the $n$th moment with respect to $x$ of this functions is a polynomial in $\eta$ of order $n+1$. This is ensured by the GPD support, which can be implemented in a simple manner by the so-called double distribution (DD) representation [1, 2]

$$
H^{i / \mathrm{A}}\left(x, \eta, \Delta^{2}, \mathcal{Q}^{2}\right)=\int_{-1}^{1} d y \int_{-1+|y|}^{1-|y|} d z x \delta(x-y-\eta z) h^{i / \mathrm{A}}\left(y, z, \Delta^{2}, \mathcal{Q}^{2}\right) .
$$

Note that, in contrast to the common definition, we included here an extra factor $x$, which guarantees that the term of order $\eta^{n+1}$ is not absent in the $n$th $x$-moment. Moreover, time reversal invariance combined with hermiticity requires that they are even in $\eta$ and, thus, the double distribution $h$ is even in $z$. The polynomiality condition is separately ensured for the two regions 
$y \geq 0$ and $y \leq 0$. The so-called positivity constraints, mentioned before, are not implemented in this representation.

For $\Delta \rightarrow 0$ the GPD (49) reduces to the parton and anti-parton distributions

$$
\left\{\begin{array}{l}
q^{i / \mathrm{A}} \\
\bar{q}^{i / \mathrm{A}}
\end{array}\right\}\left(x, \mathcal{Q}^{2}\right)=\left\{\begin{array}{c}
H^{i / \mathrm{A}}\left(x, \eta=0, \Delta^{2}=0, \mathcal{Q}^{2}\right) \\
-H^{i / \mathrm{A}}\left(-x, \eta=0, \Delta^{2}=0, \mathcal{Q}^{2}\right)
\end{array}\right\}
$$

in a nucleus with the familiar interpretation. Both of them have the support $0 \leq x \leq 1$ and we applied here the standard sign conventions for anti-quarks, so that $\bar{q}^{i / \mathrm{A}}$ is positive. They satisfy the charge sum rule

$$
\begin{aligned}
\int_{0}^{1} d x \sum_{i=u, d, s} Q_{i}\left[q^{i / \mathrm{A}}\left(x, \mathcal{Q}^{2}\right)-\bar{q}^{i / \mathrm{A}}\left(x, \mathcal{Q}^{2}\right)\right] & =\int_{0}^{1} d x\left[Q_{u} q^{u_{\text {val }} / \mathrm{A}}\left(x, \mathcal{Q}^{2}\right)+Q_{d} q^{d_{\text {val }} / \mathrm{A}}\left(x, \mathcal{Q}^{2}\right)\right] \\
& =Z
\end{aligned}
$$

and the Baryon number conservation sum rule

$$
\begin{aligned}
\int_{0}^{1} d x \frac{1}{3} \sum_{i=u, d, s}\left[q^{i / \mathrm{A}}\left(x, \mathcal{Q}^{2}\right)-\bar{q}^{i / \mathrm{A}}\left(x, \mathcal{Q}^{2}\right)\right] & =\int_{0}^{1} d x \frac{1}{3}\left[q^{u_{\mathrm{val}} / \mathrm{A}}\left(x, \mathcal{Q}^{2}\right)+q^{d_{\text {val }} / \mathrm{A}}\left(x, \mathcal{Q}^{2}\right)\right] \\
& =A
\end{aligned}
$$

In both sum rules the sea quarks drop out and we can read off the number of valence quarks

$$
\begin{aligned}
& \int_{0}^{1} d x q^{u_{\text {val }} / \mathrm{A}}\left(x, \mathcal{Q}^{2}\right)=N_{u_{\text {val }}}=2 Z+N, \\
& \int_{0}^{1} d x q^{d_{\text {val }} / \mathrm{A}}\left(x, \mathcal{Q}^{2}\right)=N_{d_{\text {val }}}=Z+2 N, \quad N=A-Z .
\end{aligned}
$$

Furthermore, we have the momentum sum rule:

$$
\int_{0}^{1} d x x \sum_{i=u, d, s}\left[q^{i / \mathrm{A}}\left(x, \mathcal{Q}^{2}\right)+\bar{q}^{i / \mathrm{A}}\left(x, \mathcal{Q}^{2}\right)\right]+\int_{0}^{1} d x x g^{\mathrm{A}}\left(x, \mathcal{Q}^{2}\right)=1,
$$

where $g^{\mathrm{A}}\left(x, \mathcal{Q}^{2}\right)$ is the gluon density.

Now we like to connect the nucleus parton distributions to the proton ones. Isospin symmetry breaking effects are rather small and, thus, we employ this symmetry to connect their parton content:

$$
\begin{aligned}
& q^{u / p}=q^{d / n} \equiv q^{u}, \quad \bar{q}^{u / p}=\bar{q}^{d / n} \equiv \bar{q}^{u}, \quad q^{d / p}=q^{u / n} \equiv q^{d}, \quad \bar{q}^{d / p}=\bar{q}^{u / n} \equiv \bar{q}^{d} \\
& q^{s / p}=\bar{q}^{s / p}=q^{s / n}=\bar{q}^{s / n} \equiv q^{s}, \quad g^{p}=g^{n} \equiv g .
\end{aligned}
$$

Considering the nucleus as a system of almost free nucleons, we can immediately express its parton distributions by those of the proton

$$
\begin{aligned}
& q^{u / \mathrm{A}}=Z q^{u}+N q^{d}, \quad q^{d / \mathrm{A}}=Z q^{d}+N q^{u}, \\
& q^{s / \mathrm{A}}=(Z+N) q^{s}, \quad g^{\mathrm{A}}=(Z+N) g .
\end{aligned}
$$


Additionally, we have to relate the momentum fraction variable $x$ to that of the struck nucleon. Since the momentum of each nucleon is the $1 /$ th part of the nucleus one, the appropriate scaling of the momentum fraction gives for instance

$$
q^{u / \mathrm{A}}(x)=A \theta(1-|x A|)\left[Z q^{u}(x A)+N q^{d}(x A)\right]
$$

and analogous for the other parton distribution. Here the loss in phase-space due to the restriction $x \leq 1 / A$ is compensated by the prefactor $A$. Note that $q^{u / A}(x)$ does in reality not vanish at $x=1 / A$, however, this simple prescription is for our purpose justified as long as $x_{\mathrm{B}}=A x_{A}$ is in the valence quark region or below. It is easy to convince ourself that all sum rules (52 53155) will be obeyed, if they are satisfied for the nucleon. For the ratio of the structure functions $F_{2}$, normalized per nucleon, in deep inelastic scattering one finds for an isoscalar target $(N=Z)$

$$
\frac{F_{2}^{\mathrm{A}}\left(x_{\mathrm{A}}\right)}{F_{2}^{\mathrm{N}}\left(x_{\mathrm{B}}\right)} \simeq \frac{1}{A} \frac{x_{\mathrm{A}} \sum_{i} Q_{i}^{2} q^{i / \mathrm{A}}\left(x_{\mathrm{A}}\right)}{x_{\mathrm{B}} \sum_{i} Q_{i}^{2} q^{i / N}\left(x_{\mathrm{B}}\right)}=1, \quad \text { with } \quad x_{\mathrm{A}}=x_{\mathrm{B}} / A
$$

Certainly, experimental measurements show that this is only true for $x_{\mathrm{B}} \sim 0.25$ and in other regions one finds characteristic deviations from this ratio, which reflect different aspects of the binding forces between the nucleons. For the kinematics we are interested in, i.e., $x_{\mathrm{B}} \sim 0.1$, it is a small difference of the order of a few percent, which can safely be neglected for our purpose.

Inspired by the parton picture, we introduce a terminology for the GPDs. However, we remind that we deal now with a generalization of distribution amplitudes and the probabilistic interpretation arises only in the forward limit, because of the optical theorem. We uniquely separate a GPD in valence- and sea-like ones

$$
H^{i / \mathrm{A}}(x, \eta)=\theta(x \geq-|\eta|) H^{i_{\mathrm{val}} / \mathrm{A}}(x, \eta)+H^{i_{\mathrm{sea}} / \mathrm{A}}(x, \eta) .
$$

The sea-like GPD is antisymmetric in $x$ and can be separated in quark and anti-quark ones. The latter are obtained from Eq. (50) due to the restriction $y \leq 0$ and the whole sea might be expressed by the anti-quark contribution

$$
H^{i_{\mathrm{sea}} / \mathrm{A}}(x, \eta)=\bar{H}^{i / \mathrm{A}}(x, \eta)-\bar{H}^{i / \mathrm{A}}(-x, \eta) .
$$

The valence-like part follows by subtracting the sea quark GPD from the whole one:

$$
H^{i_{\mathrm{val}} / \mathrm{A}}(x, \eta)=\theta(x \geq-|\eta|)\left[H^{i / \mathrm{A}}(x, \eta)-H^{i_{\mathrm{sea}} / \mathrm{A}}(x, \eta)\right] .
$$

Note that the valence-like GPDs have no symmetry property and that we included the region $-|\eta| \leq x \leq|\eta|$ in our definitions, although their partonic interpretation belongs more to the excitation of a mesonic like state. However, Lorentz invariance requires that the 'inclusive' region, which is closer to a probabilistically partonic interpretation, is entirely determined by the 'exclusive' one - the reverse is generally not true.

Let us now discuss the form factor aspects of GPDs. We define for any parton species its own partonic form factor, which is given by the lowest moment:

$$
\int_{-\xi}^{1} d x H^{i / \mathrm{A}}\left(x, \eta, \Delta^{2}, \mathcal{Q}^{2}\right)=N_{i} F^{i}\left(\Delta^{2}\right) .
$$


The above given definition (59) induces the equality of sea- and anti-quark form factors. Moreover, Lorentz invariance and current conservation ensure that the r.h.s. is independent on $\eta$ and $\mathcal{Q}^{2}$, respectively. We normalize the partonic form factors to one

$$
F^{i}\left(\Delta^{2}=0\right)=1
$$

Thus, $N_{i}$ might be interpreted as the number of a given quark species $i$ inside the nucleus.

The above given sum rules for parton densities can now be generalized to the non-forward kinematics. The charge sum rule (52) turns into the relation between partonic and electromagnetic form factors:

$$
F\left(\Delta^{2}\right)=Q_{u} N_{u_{\mathrm{val}}} F^{u_{\mathrm{val}}}\left(\Delta^{2}\right)+Q_{d} N_{d_{\mathrm{val}}} F^{d_{\mathrm{val}}}\left(\Delta^{2}\right), \quad F\left(\Delta^{2}=0\right)=Z,
$$

while the Baryon conserving ones (53) reads

$$
F^{\mathrm{Bar}}\left(\Delta^{2}\right)=\frac{1}{3} N_{u_{\mathrm{val}}} F^{u_{\mathrm{val}}}\left(\Delta^{2}\right)+\frac{1}{3} N_{d_{\mathrm{val}}} F^{d_{\mathrm{val}}}\left(\Delta^{2}\right), \quad F^{\mathrm{Bar}}\left(\Delta^{2}=0\right)=A,
$$

The sea-like GPDs are antisymmetric in $x$ by definition and so they can not contribute to these sum rules. The first moment of the singlet combination, including the gluonic component,

$$
\int_{-1}^{1} d x x\left[\sum_{i=u, d, s} H^{i / \mathrm{A}}\left(x, \eta, \Delta^{2}, \mathcal{Q}^{2}\right)+H^{g / \mathrm{A}}\left(x, \eta, \Delta^{2}, \mathcal{Q}^{2}\right)\right]=\frac{1}{P_{+}^{2}}\left\langle\mathrm{~A}\left(P_{2}\right)\left|T_{++}\right| \mathrm{A}\left(P_{1}\right)\right\rangle
$$

is given by the expectation value of the +- components of the energy-momentum tensor $T_{\mu \nu}$. Since of current conservation, it is also independent on the scale $\mathcal{Q}$. The r.h.s. is then a polynomial in $\eta$ of the second order whose coefficients are given by the two gravitational form factors, which appear in the matrix element $\left\langle\mathrm{A}\left(P_{2}\right)\left|T_{\mu \nu}\right| \mathrm{A}\left(P_{1}\right)\right\rangle$ :

$$
\frac{1}{P_{+}^{2}}\left\langle\mathrm{~A}\left(P_{2}\right)\left|T_{++}\right| \mathrm{A}\left(P_{1}\right)\right\rangle=T^{1}\left(\Delta^{2}\right)+\eta^{2} T^{2}\left(\Delta^{2}\right), \quad \text { with } \quad T^{1}\left(\Delta^{2}=0\right)=1 .
$$

The normalization of $T^{1}\left(\Delta^{2}=0\right)$ is consistent with the momentum sum rule (55). It has been argued in Ref. [36] that the second form factor is related to the spatial distribution of strong forces inside a nucleus and that its partonic content can be accessed via the GPDs.

To relate the nucleus GPDs to those of the proton, we rescale, as in the forward case, the longitudinal momentum fractions, i.e., their $x$-dependence and $\eta$-dependence. This is again motivated by considering the nucleus as a system of almost free nucleons, which have approximately the same momenta $P_{1} / A=(1-\eta) P_{+} / 2 A$. At leading order in $1 / Q^{2}$, the interaction with the two photons take then place on one of them, which has the outgoing momentum $P_{1} / A+\Delta=(1-\eta+2 A \eta) P_{+} / 2 A+\Delta^{\perp}$. Thus, instead of the GPD definition (49) we have to consider the matrix element, see Fig. 3 a

$$
\left.\int \frac{d \kappa}{2 \pi} e^{i \kappa P_{+} x}\left\langle N\left(P_{1} / A+\Delta\right)\left|\bar{\psi}_{i}(-\kappa n) \gamma_{+} \psi_{i}(\kappa n)\right| N\left(P_{1} / A\right)\right\rangle\right|_{\eta=\frac{\Delta_{+}}{P_{+}}} .
$$

To make contact with the nucleon GPD, we refer in this matrix element to the light-cone component of the struck nucleon $P_{+}^{N}=[1-\eta+A \eta] P_{+} / A$. This implies the rescaling prescription for the light-cone momentum fraction and skewedness parameter

$$
x_{N}=\frac{1-\eta_{N}}{1-\eta} A x \quad \text { and } \quad \eta_{N} \equiv \frac{\Delta_{+}}{P_{+}^{N}}=\frac{1-\eta_{N}}{1-\eta} A \eta
$$




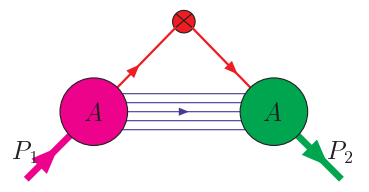

(a)

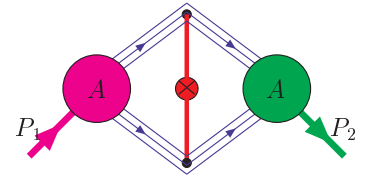

(b)

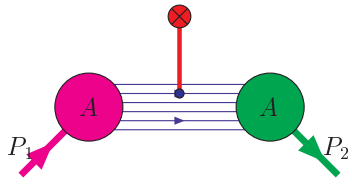

(c)

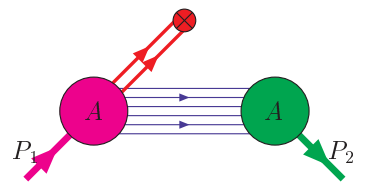

(d)

Figure 3: Different contributions to a nucleus GPD in which the light-ray operator couples to a nucleon (a), meson exchange in the $t$-channel (b), mesonic contact term (c), and virtual nucleon pair (d).

From the second equality one easily recovers the scaling law for the kinematical variable, namely, $x_{\mathrm{B}}=A x_{\mathrm{A}}$. However, this picture can not be quite realistic, since transferring the whole transversal momentum $\Delta^{\perp}$ to the struck nucleon, should, compared to a binding energy of about $10 \mathrm{MeV}$ per nucleon, induce target dissociation. On the other hand, nuclei form factors are non-zero even for rather large momentum transfer, which shows that there is a certain probability that the transversal momentum transfer is almost equally distributed among the constituents ${ }^{5}$. Assuming that the $\Delta^{2}$-dependences factorize, we can take care of this bound state effect by replacing the partonic proton form factors with the nucleus ones:

$$
\begin{aligned}
H^{u_{\mathrm{val}} / \mathrm{A}}\left(x, \eta, \Delta^{2}\right) & =F^{u_{\mathrm{val}} / \mathrm{A}}\left(\Delta^{2}\right)\left|\frac{d x_{N}}{d x}\right| \theta\left(\left|x_{N}\right| \leq 1\right)\left[Z H^{u_{\mathrm{val}}}+N H^{d_{\mathrm{val}}}\right]\left(x_{N}, \eta_{N}\right), \\
H^{d_{\mathrm{val}} / \mathrm{A}}\left(x, \eta, \Delta^{2}\right) & =F^{d_{\mathrm{val}} / \mathrm{A}}\left(\Delta^{2}\right)\left|\frac{d x_{N}}{d x}\right| \theta\left(\left|x_{N}\right| \leq 1\right)\left[Z H^{d_{\mathrm{val}}}+N H^{u_{\mathrm{val}}}\right]\left(x_{N}, \eta_{N}\right), \\
H^{i_{\mathrm{sea}} / \mathrm{A}}\left(x, \eta, \Delta^{2}\right) & =F^{\text {sea } / \mathrm{A}}\left(\Delta^{2}\right)\left|\frac{d x_{N}}{d x}\right| \theta\left(\left|x_{N}\right| \leq 1\right) A H^{i_{\text {sea }}}\left(x_{N}, \eta_{N}\right), \\
H^{g / \mathrm{A}}\left(x, \eta, \Delta^{2}\right) & =F^{g / \mathrm{A}}\left(\Delta^{2}\right)\left|\frac{d x_{N}}{d x}\right| \theta\left(\left|x_{N}\right| \leq 1\right) A H^{g}\left(x_{N}, \eta_{N}\right) .
\end{aligned}
$$

Here we introduced the reduced proton GPDs by $H^{i}(x, \eta)=H^{i}\left(x, \eta, \Delta^{2}=0\right)$. The support of the proton GPDs induces the restriction $A|x| \leq(1-\eta) /\left(1-\eta_{N}\right)$, which of course is only an artifact of our approximation. It is easy to check that by construction all sum rules remain valid. For an isoscalar target isospin symmetry requires

$$
H^{u / \mathrm{A}}\left(x, \eta, \Delta^{2}\right)=H^{d / \mathrm{A}}\left(x, \eta, \Delta^{2}\right)
$$

and, thus, also the partonic $u$ and $d$ form factors are equal.

Obviously, what we presented here is the simplest version of a convolution model for nuclei GPDs, which certainly can be improved by incorporation of binding effects. Hopefully, they might provide only small corrections to the scaling law (70) and should explain the distribution of transversal momentum transfer. Nevertheless, in the case that such effects are responsible for

\footnotetext{
${ }^{5}$ Certainly, it remains an experimental problem to ensure that the measured Compton scattering process is coherent and a theoretical task to derive the predictions for target dissociation.
} 
symmetry breaking, e.g., the breaking of spherical symmetry implies a non-vanishing quadrupole moment for deuteron, they might become a leading contribution to GPDs that decouples in the forward case. Thus, it is worth to have a closer look on them.

First we consider the possibility that the light-ray operator insertion couples to meson exchange currents in the $t$-channel, as it is shown in Fig. $3 \mathrm{~b}$. The corresponding matrix element reads

$$
\begin{aligned}
& \left.\int \frac{d \kappa}{2 \pi} e^{i \kappa P_{+} x}\left\langle n\left(P_{1} / A+\Delta / 2\right) p\left(P_{1} / A+\Delta / 2\right)\left|\bar{\psi}_{i}(-\kappa n) \gamma_{+} \psi_{i}(\kappa n)\right| p\left(P_{1} / A\right) n\left(P_{1} / A\right)\right\rangle\right|_{\eta=\frac{\Delta_{+}}{P_{+}}} ^{t-\text { channel }} \\
& \left.\propto \int_{-1}^{1} d u \phi(u)\left|\frac{u d x^{\mathrm{ex}}}{d x}\right| \int \frac{d \kappa}{2 \pi} e^{i \kappa P_{+}^{\mathrm{ex}} x^{\mathrm{ex}}}\langle\pi([u-1] \Delta / 2)|\mathcal{O}(\kappa,-\kappa)| \pi([u+1] \Delta / 2)\rangle\right|_{\eta^{\mathrm{ex}}=\frac{\Delta_{+}}{P_{+}^{\mathrm{ex}}}} .
\end{aligned}
$$

Here the probability amplitude $\phi(u)$ with $|u| \leq 1$ is a function of the light-cone momentum fraction $u$, defined by $P_{+}^{\mathrm{ex}}=u \Delta_{+}=u \eta P_{+}$. The momentum fraction $x^{\mathrm{ex}}$ and skewedness parameter $\eta^{\mathrm{ex}}$, entering in the GPD, are

$$
x^{\mathrm{ex}}=\frac{x}{u \eta} \quad \text { and } \quad \eta^{\mathrm{ex}} \equiv \frac{\Delta_{+}}{P_{+}^{\mathrm{ex}}}=\frac{1}{u} .
$$

The support property of the resulting GPD

$$
H^{t-\text { cannel }}(x, \eta) \propto \frac{x}{|\eta|} \int_{-1}^{1} d u \operatorname{sign}(u \eta) \phi(u) \int_{-1}^{1} d y \int_{-1+|y|}^{1-|y|} d z \delta(x-\eta[u y+z]) F_{\pi}^{t-\text { cannel }}(y, z)
$$

can be deduced from the DD representation. It only contributes in the 'exclusive' region, since for the 'inclusive' region we find $|x / \eta|>1 \geq|u y+z|$. Time-reversal invariance combined with hermiticity requires that $\phi$ and $F_{\pi}^{t-\text { cannel }}(y, z)$ are symmetric in $u$ and $z$, respectively. Consequently, the exchange GPD is also symmetric in $x$ and the polynomiality condition is satisfied in general. For the lowest moment we have the normalization:

$$
\int_{-1}^{1} d x H^{t-\text { cannel }}(x, \eta) \propto \int_{-1}^{1} d u|u| \phi(u) \int_{-1}^{1} d y y \int_{-1+|y|}^{1-|y|} d z F_{\pi}^{t-\text { cannel }}(y, z) .
$$

Remembering that the integral over $z$ gives the parton densities of the pion, i.e., $q_{\pi}(y) / y$, we obviously, establish a link between 'exclusive' $t$-channel contributions and the parton picture. Again the total sea quark contribution $q_{\pi}^{\text {sea }}(y)$ is antisymmetric in $y$ and, thus, it drops out in the sum rules (6465) and also for higher moments (which are even). Such an $I=1$ isospin exchange contribution is 'filtered' out for an isoscalar target, however, it might be important for other targets or dissociation processes. Note that for an isoscalar target, resonance exchange contributions appear, like the famous $\rho \pi \gamma$ exchange current. We should add that in such a case also contact terms, depicted in Fig. 3r, have to be included, which for instance are given by

$$
\left.\int \frac{d \kappa}{2 \pi} e^{i \kappa P_{+} x}\left\langle N\left(P_{1} / A+\Delta / 2\right) \pi(\Delta / 2)\left|\bar{\psi}_{i}(-\kappa n) \gamma_{+} \psi_{i}(\kappa n)\right| N\left(P_{1} / A\right)\right\rangle\right|_{\eta=\frac{\Delta_{+}}{P_{+}}} .
$$

Moreover, Lorentz invariance requires that virtual nucleus anti-nucleus states contribute to the nucleon GPD. An example is depicted in Fig. 3 3 and its matrix element reads

$$
\left.\int \frac{d \kappa}{2 \pi} e^{i \kappa P_{+} x}\left\langle\Omega\left|\bar{\psi}_{i}(-\kappa n) \gamma_{+} \psi_{i}(\kappa n)\right| N\left(p_{1}\right) \bar{N}\left(p_{2}\right)\right\rangle\right|_{\eta=\frac{\Delta_{+}}{P_{+}}}
$$


It is far beyond the scope of this paper to establish a closer link between the effective forces of nuclei and the fundamental degree of freedom in QCD, however, our discussion shows that GPDs are an appropriate tool for this task.

Based on a 'popular' model for the reduced proton GPDs, we present now our model for a scalar nucleus. To implement the support properties we deal with the DD representation [1, 2] at a given input scale $\mathcal{Q}=\mathcal{Q}_{0}$ :

$$
H^{i}(x, \eta)=\int_{-1}^{1} d y \int_{-1+|y|}^{1-|y|} d z \delta(x-y-\eta z) q^{i}(y) \pi\left(|y|, z ; b^{i}\right)+\operatorname{sign}(\eta) D^{i}(x / \eta)
$$

and adopt Radyushkin's proposal for the factorization of the DD into a forward parton density $q^{i}(y)$ given at the input scale $\mathcal{Q}_{0}$ and a profile function

$$
\pi(y, z ; b)=\frac{\Gamma\left(b+\frac{3}{2}\right)}{\sqrt{\pi} \Gamma(b+1)} \frac{\left[(1-y)^{2}-z^{2}\right]^{b}}{(1-y)^{2 b+1}},
$$

which is normalized to 1 :

$$
\int_{-1+|y|}^{1-|y|} d z \pi(|y|, z ; b)=1
$$

Here the free parameter $b$ models the skewedness effect that arises from the $x$-shape of GPDs for a given value of $\eta$. In the limit $b \rightarrow \infty$ the GPDs are independent of $\eta$ and are simply given by $q^{i}(x)$ itself. For the unpolarized parton densities of the proton we will take the MRS A' parameterization [46], given at the input scale $\mathcal{Q}_{0}^{2}=4 \mathrm{GeV}^{2}$, with $\bar{u}=\bar{d}=\bar{s} / 2$. For the electromagnetic form factors we adopt here for simplicity the following parameterization

$$
F^{u_{\mathrm{val}} / \mathrm{A}}\left(\Delta^{2}\right)=F^{d_{\mathrm{val}} / \mathrm{A}}\left(\Delta^{2}\right)=\frac{1}{Z} F^{\mathrm{A}}\left(\Delta^{2}\right), \quad F^{\text {sea } / \mathrm{A}}\left(\Delta^{2}\right)=e^{B^{\mathrm{sea}} A^{2 / 3} \Delta^{2}}
$$

where the slope $A^{2 / 3} B^{\text {sea }}$ of the sea quarks is defined in such a way that $B^{\text {sea }}$ only weakly depends on $A$. This is motivated by the fact that the nucleus density is rather independent on $A$ and, thus, we expect that the radius of the sea quark distribution should scale with $A^{1 / 3}$. For the electromagnetic form factor we adopt the parameterization of Ref. [47:

$$
F^{\mathrm{A}}\left(\Delta^{2}\right)=Z\left(1-\frac{1}{6} c^{2} \Delta^{2}\right)^{-1} \exp \left\{\frac{1}{6} \Delta^{2} b^{2}\right\}
$$

with $b=2.04 \times 5.07 \mathrm{GeV}^{-1}$ and $c=1.07 A^{1 / 3} \times 5.07 \mathrm{GeV}^{-1}$.

There are a few comments in order. First the factorization in $\Delta^{2}$ for medium values has no theoretical or experimental justifications. In comparison with experimental data, which are presently measured at a mean value of $<-\Delta^{2}>\sim 0.1 \ldots 0.3 \mathrm{GeV}^{2}$, the $\Delta^{2}$-dependences effectively enters only the normalization of the CFFs via the slope parameters. Second remark concerns the polynomiality property of the moments. In comparison to Eq. (50) we choose for simplicity another DD representation in Eq. (177). The prefactor $x$ is now neglected and the highest possible term $\eta^{n+1}$ for the $n$th odd $x$-moment is restored by the so-called $D$-term. For detailed discussions on this subject see Ref. [4, 6]. This $D$-term is antisymmetric in $x$ and can not spoil the sum rule 
(62), however, it enter the sum rule (67) and is discussed below. In our language we count it as a sea-quark contribution. The third remark concerns the factorization of the DDs ansatz, that is inspired by an intuitive picture and provide in general an enhancement at the point $x= \pm \eta$. Finally, we should comment on the meson exchange contributions. For a scalar target we will not explicitly take them into account. They effectively enter here the parameterization of the form factors. Moreover, they have do vanish in the forward limit and, therefore, they should be suppressed for valence like contributions at small $\Delta^{2}$, while for sea-like contributions they can be included in the antisymmetric $D$-term.

Let us finish this section by expressing the nucleus $\mathrm{CFF} \mathcal{H}^{\mathrm{A}}$ by the proton ones in the twist-two and twist-three sector. From the ansatz (170) and the convolution ${ }^{6}$ (35), one easily establish the following scaling law for the twist-two CFF of a isoscalar nucleus

$$
\mathcal{H}^{\mathrm{A}}=A^{2} \frac{1+\xi_{N}}{1+\xi}\left[\frac{Q_{u}^{2}+Q_{d}^{2}}{2 Z} F^{\mathrm{A}}\left(\Delta^{2}\right)\left(\frac{\mathcal{H}^{u_{\mathrm{val}}}}{Q_{u}^{2}}+\frac{\mathcal{H}^{d_{\text {val }}}}{Q_{d}^{2}}\right)\left(\xi_{N}\right)+F^{\text {sea } / \mathrm{A}}\left(\Delta^{2}\right) \mathcal{H}^{\text {sea }}\left(\xi_{N}\right)\right] .
$$

Here $\mathcal{H}^{i}\left(\xi_{N}\right)$ denotes the contribution that appears in the decomposition of the proton $\mathrm{CFF}$ $\mathcal{H}=\mathcal{H}^{u_{\text {val }}}+\mathcal{H}^{d_{\text {val }}}+\mathcal{H}^{\text {sea }}$ at $\Delta^{2}=0$. Note that, as long we rely on the ansatz (70), this scaling law is valid beyond the LO approximation, since the hard-scattering coefficients have the following functional dependence $1 / \xi C(x / \xi)$. It is interesting to note that in Ref. [36], it was argued that the $D$-term contribution to the Compton form factor, which is independent of $x_{\mathrm{B}}$, scales with $A^{7 / 3}$. Its contribution to the second gravitational form factor is

$$
T^{2}\left(\Delta^{2}\right)=\frac{1}{2} \frac{d^{2}}{d \eta^{2}} \sum_{i=u, d, s, g} \int_{-1}^{1} d x x H^{i / \mathrm{A}}\left(x, \eta, \Delta^{2}\right)=\frac{4}{5} A^{2} d^{\mathrm{A}}\left(\Delta^{2}\right) .
$$

To study the $D$-term with respect to $A$ and in relation to the sea quark contribution per nucleon, we have rescaled $d^{\mathrm{A}}$ by $A^{2}$. Based on the estimate in the same reference, one finds that $d^{\mathrm{A}}\left(\Delta^{2}=0\right)$ slightly increases with $A \geq 8$ :

$$
\begin{aligned}
d^{\mathrm{A}}\left(\Delta^{2}=0\right) & =d^{Q / \mathrm{A}}\left(\Delta^{2}=0\right)+d^{G / \mathrm{A}}\left(\Delta^{2}=0\right) \approx-0.2 A^{1 / 3}\left(1+3.8 / A^{2 / 3}\right), \\
d^{Q / N}\left(\Delta^{2}=0\right) & \approx-4.0 \text { at } \mu \approx 0.6 \mathrm{GeV},
\end{aligned}
$$

where the second result for the nucleon $D$-term in the quark sector has been predicted by a model calculation within the chiral soliton model [49, 50. As it will be discussed in Section 5.1, a present measurement of the charge asymmetry on the proton target does not allow for a definite conclusion of the $D$-term contribution. Unfortunately, we might conclude that for nucleus target, e.g., $d^{\mathrm{A}} \approx-1.3$ for $A=200$, this term will not induce a significant contribution, too. Certainly, it is an important task to confront this expectation with experimental measurements.

Employing the equation of motion, the twist-tree GPDs are decomposed in the so called Wandzura-Wilczek (WW) term, entirely expressed by the twist-two GPD $H$, and the quarkgluon-quark GPD $H^{\mathrm{qGq}}$, carrying new dynamical information. The two twist-three GPDs enter in the DVCS amplitude always in the same linear combination [6]

$$
\mathcal{H}^{\mathrm{eff}}=\mathcal{H}^{\mathrm{eff}-\mathrm{WW}}+\mathcal{H}^{\mathrm{qGq}}
$$

\footnotetext{
${ }^{6}$ Remove the index $k$ for a scalar target .
} 
where

$$
\begin{aligned}
& \mathcal{H}^{A \mathrm{eff}-\mathrm{WW}}=\frac{2}{1+\xi} \mathcal{H}^{\mathrm{A}}+2 \xi \frac{\partial}{\partial \xi} \sum_{i=u, d, s} \int_{-1}^{1} d x \frac{Q_{i}^{2}}{\xi+x} \ln \frac{2 \xi}{\xi-x-i 0} \\
& \times\left\{H^{i / \mathrm{A}}(x, \xi)-H^{i / \mathrm{A}}(-x, \xi)\right\} .
\end{aligned}
$$

Using the ansatz (170), one easily derives the WW piece in terms of the proton GPDs $H^{i}$. For instance, the total sea quark contribution reads in terms of the $\mathrm{SU}(2)$ symmetric antiquark GPDs

$$
\begin{aligned}
& \frac{2}{1+\xi} \mathcal{H}^{\mathrm{sea} / \mathrm{A}}\left(\xi, \Delta^{2}\right)+A^{2} F^{\mathrm{sea} / \mathrm{A}}\left(\Delta^{2}\right) 2 \xi \frac{\partial}{\partial \xi} \frac{1+\xi_{N}}{1+\xi} \sum_{i=u, d, s} \int_{-1}^{1} d x \frac{Q_{i}^{2}}{\xi_{N}+x} \ln \frac{2 \xi_{N}}{\xi_{N}-x-i 0} \\
& \times 2\left\{\bar{H}^{i / \mathrm{A}}\left(x, \xi_{N}\right)-\bar{H}^{i / \mathrm{A}}\left(-x, \xi_{N}\right)\right\} \text {, }
\end{aligned}
$$

where $\xi_{N}=A \xi /(1+\xi-A \xi)$ is to be considered as a function of $\xi$. The analogous formula in the valence quark sector follows by an appropriate replacement of form factor and GPDs together with the corresponding charge factors.

\subsection{GPDs of spin-1/2 nucleus target}

Analogous models for proton GPDs have been used in Ref. 7], for a detailed discussion of GPDs see Ref. [51, and they are consistent with all experimental data available at present in the LO analysis. It has been stressed in Ref. [52 that this not necessarily true in NLO. The problem arises from the employed model of the flavour singlet GPDs, especially from the gluonic ones. We will skip this issue here and refer to the discussion in Ref. [7. Due to the spin structure of the nucleon, one has to model four different sets of GPDs:

$$
F=\{H, E, \widetilde{H}, \widetilde{E}\}
$$

corresponding to helicity conserved and non-conserved form factors with even and odd parity. Note that for an unpolarized target the dominant contribution arises from the helicity conserved and parity even GPDs $H^{i}$. They satisfy the constraints

$$
\lim _{\Delta \rightarrow 0} H^{i}\left(x, \eta, \Delta^{2}\right)=q^{i}(x) \text { and } \int_{-1}^{1} d x H^{i}\left(x, \eta, \Delta^{2}\right)=F_{1}^{i}\left(\Delta^{2}\right)
$$

For longitudinally polarized target both $H$ and $\widetilde{H}$ are important. The latter ones are related to the polarized sea quark content and axial-vector form factors:

$$
\lim _{\Delta \rightarrow 0} \widetilde{H}^{i}\left(x, \eta, \Delta^{2}\right)=\Delta q^{i}(x) \text { and } \int_{-1}^{1} d x \widetilde{H}^{i}\left(x, \eta, \Delta^{2}\right)=G_{1}^{i}\left(\Delta^{2}\right)
$$

respectively. The helicity non-conserved GPDs $E$ and $\tilde{E}$, which decouple in the forward limit, obey the sum rules

$$
\int_{-1}^{1} d x E^{i}\left(x, \eta, \Delta^{2}\right)=F_{2}^{i}\left(\Delta^{2}\right) \quad \text { and } \quad \int_{-1}^{1} d x \widetilde{E}^{i}\left(x, \eta, \Delta^{2}\right)=G_{2}^{i}\left(\Delta^{2}\right) .
$$


Perhaps they can be accessed in transversally polarized target experiments. An essential observation was that the ansatz (77) for the sea-quarks, measured in the small $x_{\mathrm{B}}$ region, has to be suppressed by choosing a $b^{\text {sea }} \rightarrow \infty$ and a large slope $B^{\text {sea }} \sim 9 \mathrm{GeV}^{2}$. This suppression then ensures also the correct size of the single beam spin asymmetry measured in fixed target experiments. For instance, the mean value at HERMES is $x_{\mathrm{B}} \approx 0.1$ and, thus, the value of $\xi \approx 0.05$ appearing in the argument of GPDs is rather small. As we said, the contribution of sea quarks remains relatively small with respect to the valence like ones. If this observation is implemented in the correct way within our GPD model remains an open issue at present.

The helicity conserved GPDs $H^{i / \mathrm{A}}$ and $\widetilde{H}^{i / \mathrm{A}}$ of a nucleus with spin-1/2 can be obtained from those of a proton by means of Eq. (170). Unfortunately, not too much is known about the structure of $E^{i / \mathrm{A}}$ and $\widetilde{E}^{i / \mathrm{A}}$, beside the pion-pole contribution of $\widetilde{E}^{i / \mathrm{A}}$. For the parametrization of the twistthree GPDs see Refs. [53, 7].

\subsection{GPDs of spin-one target}

In this section we list the properties that are known about the spin-one GPDs and have been already given in Ref. 32. For the matrix element of the twist-two operators we use the analogous kinematical decomposition as for the CFFs in Eqs. (33) and (34). Combining hermiticity and time reversal invariance tells us that the GPDs are real valued functions, which respect the symmetry properties:

$$
\begin{array}{lll}
H_{k}(x, \eta)=H_{k}(x,-\eta) & \text { for } k=\{1,2,3,5\}, & H_{4}(x, \eta)=-H_{4}(x,-\eta), \\
\widetilde{H}_{k}(x, \eta)=\widetilde{H}_{k}(x,-\eta) \text { for } k=\{1,2,4\}, & \widetilde{H}_{3}(x, \eta)=-\widetilde{H}_{3}(x,-\eta) .
\end{array}
$$

Note that $H_{4}(x, \eta)$ and $\widetilde{H}_{3}(x, \eta)$ are antisymmetric with respect to $\eta$, and, consequently, their moments are odd polynomials in $\eta$.

From the definition of GPDs as Fourier transforms of light-ray operators it follows that their lowest moment is given by the form factors appearing in the vector or axial-vector current, respectively. In the former case they are related to the electromagnetic form factors

$$
\begin{gathered}
\sum Q_{i} \int_{-1}^{1} d x H_{k}^{i / \mathrm{A}}\left(x, \eta, \Delta^{2}, \mathcal{Q}^{2}\right)=G_{k}\left(\Delta^{2}\right) \text { for } k=\{1,2,3\} \\
\int_{-1}^{1} d x H_{k}^{i / \mathrm{A}}\left(x, \eta, \Delta^{2}, \mathcal{Q}^{2}\right)=0 \text { for } k=\{4,5\}
\end{gathered}
$$

or they have to vanish. For the deuteron the electromagnetic form factors are known from experimental measurements and their parameterization is given in Appendix A [54, 55. For a target with charge $Z e$ we choose the normalization

$$
G_{1}\left(\Delta^{2}=0\right)=Z, \quad G_{2}\left(\Delta^{2}=0\right)=Z \mu_{A}, \quad G_{3}^{i}\left(\Delta^{2}=0\right)=Z\left(\mu_{A}+\mathcal{Q}_{A}-1\right),
$$

where $Z \mu_{A}$ is the magnetic moment and $Z \mathcal{Q}_{A}$ is the electrical quadrupole moment. The latter is induced by non-central nuclear forces. Its non-vanishing value, measured for the deuteron, has been considered as an evidence for the role of pions in nuclear physics. More precisely, the one pion exchange provides at larger distances the dominant contribution to the potential, which is of the Yukawa type, and induces a D-wave admixture to the S-wave function. 
The sum rules for parity odd GPDs read

$$
\begin{aligned}
& \int_{-1}^{1} d x \widetilde{H}_{k}^{i}\left(x, \eta, \Delta^{2}, \mathcal{Q}^{2}\right)=\widetilde{G}_{k}^{i}\left(\Delta^{2}\right) \text { for } k=\{1,2\} \\
& \int_{-1}^{1} d x \widetilde{H}_{k}^{i}\left(x, \eta, \Delta^{2}, \mathcal{Q}^{2}\right)=0 \text { for } k=\{3,4\}
\end{aligned}
$$

The two axial form factors $\widetilde{G}_{k}^{i}\left(\Delta^{2}\right)$ with $k=\{1,2\}$ are defined by the matrix element of the current $j_{\mu}^{5, i}=\bar{\psi}_{i}(0) \gamma_{\mu} \gamma^{5} \psi_{i}(0)$ with flavour $i$ :

$$
J_{\mu}^{5, i}=i \epsilon_{\mu \epsilon_{2}^{*} \epsilon_{1} P} \widetilde{G}_{1}\left(\Delta^{2}\right)-\frac{i \epsilon_{\mu \Delta P \epsilon_{1}} \epsilon_{2}^{*} \cdot P+i \epsilon_{\mu \Delta P \epsilon_{2}^{*}} \epsilon_{1} \cdot P}{M_{\mathrm{A}}^{2}} \widetilde{G}_{2}\left(\Delta^{2}\right),
$$

In principle they, i.e., certain linear combinations with respect to the flavour number, can be measured due to weak interaction, however, to our best knowledge this has not be done yet.

First we consider the forward limit in which $H_{2}, H_{3}, H_{4}$ as well as $\widetilde{H}_{2}, \widetilde{H}_{3}, \widetilde{H}_{4}$ decouple from the Compton amplitude and, thus, are not measurable in deep-inelastic scattering. This does not mean that the functions in question vanish by themselves. The remaining three functions are expressed in terms of parton densities

$$
\begin{aligned}
q^{i / \mathrm{A}}(x) & \equiv H_{1}^{i / \mathrm{A}}\left(x, \eta=0, \Delta^{2}=0\right)=\frac{1}{3}\left\{q^{+1}(x)+q^{-1}(x)+q^{0}(x)\right\}, \\
\delta q^{i / \mathrm{A}}(x) & \equiv H_{5}\left(x, \eta=0, \Delta^{2}=0\right)=q^{0}(x)-\frac{1}{2}\left\{q^{+1}(x)+q^{-1}(x)\right\}, \\
\Delta q^{i / \mathrm{A}}(x) & \equiv \widetilde{H}_{1}^{i / \mathrm{A}}\left(x, \eta=0, \Delta^{2}=0\right)=q_{\uparrow}^{+1}(x)-q_{\uparrow}^{-1}(x),
\end{aligned}
$$

where $q^{\Lambda}=q_{\uparrow}^{\Lambda}+q_{\downarrow}^{\Lambda}$. Here $q_{\uparrow}^{\Lambda}(x)$ is the probability to find a (anti)quark with momentum fraction $x>0(x<0)$ and positive helicity in the target of helicity $\Lambda$. Note that these definitions contain both quark $(x \geq 0)$ and antiquark $(x \leq 0)$ contributions with the following sign conventions:

$$
\bar{q}(x)=-q(-x), \quad \delta \bar{q}(x)=-\delta q(-x), \quad \Delta \bar{q}(x)=\Delta q(-x), \quad \text { for } \quad x \geq 0 .
$$

The combination of quark distributions in $H_{5}$ enter the structure function $b_{1}$, measurable in deeply inelastic scattering on a polarized spin-one target. The sum rule (98) then induces

$$
\int_{-1}^{1} d x \delta q(x)=0 \quad \Longrightarrow \quad \int_{0}^{1} d x \delta q^{\mathrm{val}}(x)=0 .
$$

To obtain the second sum rule for valence quarks we employed the antisymmetry property of the sea quarks. This relation then converts into a sum rule for $b_{1}$

$$
\int_{0}^{1} d x b_{1}(x)=2 \sum_{i=u, d, s} e_{i}^{2} \int_{0}^{1} \delta \bar{q}_{i}(x) d x,
$$

which vanishes for an unpolarized quark sea [56]. Considering the deuteron as composed of almost free nucleons induces a vanishing ratio $b_{1} / F_{1}$ [28]. The first preliminary measurement from the HERMES collaboration [57] indicates a tensor asymmetry, $A_{z z}=-2 b_{1} / 3 F_{1}$, that strongly depends 
on $x_{\mathrm{B}}$. It is compatible with zero in the valence quark region, but significantly positive and negative for large and small $x_{\mathrm{B}}$, respectively. Since we are in the following interested in the valence quark region, we set $\delta \bar{q}_{i}(x)=0$. Consequently, this simplifies the spin content of the unpolarized quark distributions

$$
q^{0}(x)=\frac{1}{2}\left\{q^{+1}+q^{-1}\right\}(x) \quad \Longrightarrow \quad q(x)=\frac{1}{2}\left\{q^{+1}+q^{-1}\right\}(x) .
$$

Let us now discuss the modelling of GPDs. In this paper we only consider numerical estimates for unpolarized or longitudinally polarized target, in which $H_{2}, H_{4}, \widetilde{H}_{2}, \widetilde{H}_{3}$, and $\widetilde{H}_{4}$ are relatively suppressed by $\xi \sim\left(x_{\mathrm{B}} / 2 A\right)$ or $\tau=\Delta^{2} / 4 A^{2} M_{N}^{2}$ in the DVCS amplitude and will be neglected. To satisfy the sum rule (95) for $H_{5}(x, \eta)$ we set it to zero. This choice is motivated by the knowledge of parton densities. Alternatively, we can also take an antisymmetric function in $x$, e.g., proportional to $H_{1}(x, \eta)-H_{1}(-x, \eta)$. It is worth noting that both GPDs can be related to the proton GPDs $E$ and $\widetilde{E}$, respectively. They are accessible in experiments with transversally polarized targets, which, however, is beyond the scope of this paper.

For the GPDs $H_{1}^{i / \mathrm{A}}$ and $\widetilde{H}_{1}^{i / \mathrm{A}}$ we employ the scaling relation (70) to connect them to the proton GPDs $H^{i}$ and $\widetilde{H}^{i}$, cf. Eqs. (56) and (57). For the latter we will neglect the D-wave admixture, which provides in the forward limit for the deuteron a few percent effect. To implement the support properties of GPDs as well as the reduction to the parton densities in a simple manner we again employ the DD representation

$$
\left\{\begin{array}{c}
H_{1}^{i} \\
\widetilde{H}_{1}^{i}
\end{array}\right\}=\left\{\begin{array}{c}
G_{1}^{i} \\
\widetilde{G}_{1}^{i}
\end{array}\right\}\left(\Delta^{2}\right) \int_{-1}^{1} d y \int_{-1+|y|}^{1-|y|} d z \delta(x-y-\eta z)\left\{\begin{array}{c}
q^{i}(y) \pi\left(|y|, z ; b_{1}^{i}\right) \\
\Delta q^{i}(y) \pi\left(|y|, z ; \widetilde{b}_{1}^{i}\right)
\end{array}\right\},
$$

where a possible $D$-term is neglected. The partonic form factors are normalized to one and for an isoscalar target we choose

$$
G_{1}^{u_{\mathrm{val}}}\left(\Delta^{2}\right)=G_{1}^{d_{\mathrm{val}}}\left(\Delta^{2}\right)=\frac{1}{Z} G_{1}\left(\Delta^{2}\right)
$$

Analogous to the scalar case, cf. Eq. (64), this choice ensures that the sum rules (94) are satisfied, where again the sea quarks do not contribute. Inspired by the counting rules, we take for the deuteron a partonic sea quark form factor with a rather simple parameterization

$$
G_{1}^{\text {sea }}\left(\Delta^{2}\right)=\left(1+\frac{2 \tau}{3} \mathcal{Q}^{\text {sea }}\right)\left(1-\frac{\Delta^{2}}{m_{\text {sea }}^{2}}\right)^{-6}
$$

where the free parameters $m_{\text {sea }}^{2}=6 / B^{\text {sea }}$, expressed by the slope $B^{\text {sea }}$ of the 'charge' form factor, and the 'quardropol' moment for sea quarks are specified below. Although theoretical considerations predict also a rather complex shape for the axial form factor, see Ref. [58] and references therein, we will use the simple parameterization

$$
\begin{aligned}
& \widetilde{G}_{1}^{i}\left(\Delta^{2}\right)=\left(1-\frac{\widetilde{B}^{i}}{5} \Delta^{2}\right)^{-5} \quad \text { for } \quad i=\left\{u^{\mathrm{val}}, d^{\mathrm{val}}\right\}, \\
& \widetilde{G}_{1}^{i}\left(\Delta^{2}\right)=\left(1-\frac{\widetilde{B}^{i}}{6} \Delta^{2}\right)^{-6} \text { for } i=\left\{u^{\text {sea }}, d^{\text {sea }}, s^{\text {sea }}\right\},
\end{aligned}
$$


for the partonic form factors in the small $-\Delta^{2}$ region. The normalization $\widetilde{G}_{1}^{i}\left(\Delta^{2}=0\right)=1$ ensures the correct reduction to the forward limit.

As mentioned above, the quadrupole moment arises from a pure bound state effect, successfully explained by the one pion exchange, and essentially given by the matrix element sandwiched between S- and D-wave state of the deuteron. As stated before, exchange and contact $I=1$ contributions drop out and so the dominant contribution should arise from the overlap

$$
\left.H_{3}^{i / d} \sim \int \frac{d \kappa}{2 \pi} e^{i \kappa P_{+} x}\left\langle d\left(P_{2},{ }^{3} D_{1}\right)\left|\bar{\psi}_{i}(-\kappa n) \gamma_{+} \psi_{i}(\kappa n)\right| d\left(P_{1},{ }^{3} S_{1}\right)\right\rangle\right|_{\eta=\frac{\Delta_{+}}{P_{+}}} .
$$

Certainly, we have to include the interaction between both nucleons. For the integrated GPD, i.e., the form factor $G_{3}$, one can write the form factor as a product of the isoscalar nucleon form factor and the so-called body form factor. The latter mainly arises due to the overlap of $D$ and $S$ waves and alters the $\Delta^{2}$ dependence of the isoscalar form factor. An extension of this approach to GPDs has been proposed in [35], where $H_{3}$ is essentially given as convolution of the isoscalar GPD, modelled as $H^{\text {iso }}=\left(H^{u}+H^{d}\right) / 2$. On the other hand, $H_{3}$ is probing the binding force of the deuteron, and as we know from deep inelastic scattering this effect rather depends on the value of $x_{\mathrm{B}}$. For GPDs the situation is rather complex and at present we have no calculation or model available that allows us to have a deeper insight in this problem. Thus, we consider two extreme cases and discuss their consequences in the next section:

- We assume that $H_{3}$ arises from the binding forces between 'partons' that carry either rather large or small momentum fractions. Thus, one might expect that such effects only slightly influence DVCS observables at present fixed target experiments. Therefore, we set for moderate values of $x_{\mathrm{B}}$ the CFF to zero:

$$
\mathcal{H}_{3}=0 \quad \text { for } \quad 0.1 \leq x_{\mathrm{B}} \leq 0.3
$$

- In the convolution model $H_{1}$ and $H_{3}$ are both essentially determined by the isoscalar GPD $H^{\text {iso }}$. So we equate the reduced GPDs $H_{1}$ and $H_{3}$ :

$$
H_{3}(x, \eta)=H_{1}(x, \eta)
$$

\section{$5 \quad$ Estimates for observables}

In Section 5.1 and 5.2 we give analytical and numerical estimates for the size of the unpolarized cross section, the beam spin asymmetry

$$
A_{\mathrm{LU}}(\phi)=\frac{d \sigma^{\uparrow}(\phi)-d \sigma^{\downarrow}(\phi)}{d \sigma^{\uparrow}(\phi)+d \sigma^{\downarrow}(\phi)},
$$

the longitudinally polarized target spin asymmetry

$$
A_{\mathrm{UL}}(\phi)=\frac{d \sigma^{\Uparrow}(\phi)-d \sigma^{\Downarrow}(\phi)}{d \sigma^{\Uparrow}(\phi)+d \sigma^{\Downarrow}(\phi)}
$$


as well as for the charge asymmetry of the unpolarized cross section

$$
A_{\mathrm{C}}(\phi)=\frac{d \sigma^{+}(\phi)-d \sigma^{-}(\phi)}{d \sigma^{+}(\phi)+d \sigma^{-}(\phi)}
$$

Moreover, for a spin-1 target we consider also the beam spin asymmetry

$$
A_{\mathrm{L} \pm}(\phi)=\frac{d \sigma^{\uparrow \Uparrow}(\phi)+d \sigma^{\uparrow \Downarrow}(\phi)-d \sigma^{\downarrow \Uparrow}(\phi)-d \sigma^{\downarrow \Downarrow}(\phi)}{d \sigma^{\uparrow \Uparrow}(\phi)+d \sigma^{\uparrow \Downarrow}(\phi)+d \sigma^{\downarrow \Uparrow}(\phi)+d \sigma^{\downarrow \Downarrow}(\phi)}
$$

and the tensor polarization

$$
A_{z z}(\phi)=\frac{d \sigma_{z z}}{3 d \sigma_{\mathrm{unp}}}, \quad d \sigma_{z z} \equiv d \sigma^{\Uparrow}+d \sigma^{\Downarrow}-2 d \sigma^{\Rightarrow}, d \sigma_{\mathrm{unp}} \equiv \frac{1}{3}\left(d \sigma^{\Uparrow}+d \sigma^{\Downarrow}+d \sigma^{\Rightarrow}\right),
$$

where $\Uparrow, \Downarrow$, and $\Rightarrow$ denote the magnetic quantum numbers $\Lambda=\{+1,-1,0\}$ for a longitudinally polarized target. We give estimates for HERMES and (upgraded) JLAB kinematics for a lepton beam of $E=27.6 \mathrm{GeV}$ and $E=6$ (12) GeV, respectively.

The single spin and charge asymmetries are dominated by the first harmonics of the interference term (27), which arise at twist-two level, while for the tensor polarization the squared BH term dominates in the charge even sector. All observables are contaminated by $O(1 / \mathcal{Q})$ suppressed effects, induced by other harmonics in both the interference and squared DVCS term or from pure kinematical effects: higher harmonics of the squared BH term (25) in the denominator and additional $\phi$ dependence due to the $\mathrm{BH}$ propagators $\mathcal{P}_{1} \mathcal{P}_{2}(\phi)$. We found that higher twist-three harmonics are small in the WW approximation [7. For the charge asymmetry also a 'constant' contribution arises in the interference term, which is completely determined by the twist-two GPDs. It can be relatively large with respect to the dominant $\cos (\phi)$ term, since in contrast to higher harmonics it does not vanish at the kinematical boundaries. Certainly, both the size of asymmetries and their power suppressed contamination depend on the ratio of DVCS to BH amplitude, which is estimated to $\mathrm{be}^{7}$

$$
\frac{\mathcal{T}^{\mathrm{DVCS}}}{\mathcal{T}^{\mathrm{BH}}} \sim \frac{A}{Z} \sqrt{\frac{-(1-y) \Delta^{2}}{y^{2} \mathcal{Q}^{2}}}
$$

For HERMES kinematics with $\left|\Delta^{2}\right|<0.3 \mathrm{GeV}^{2}, x_{\mathrm{B}} \sim 0.1$, and $\mathcal{Q}^{2} \sim 2 \mathrm{GeV}^{2}$, i.e., $y \sim 0.5$, the ratio is $\mathcal{T}^{\mathrm{DVCS}} / \mathcal{T}^{\mathrm{BH}}<0.5 A / Z$. For JLAB the value of $y$ is typically larger. This results into a stronger suppression of the DVCS amplitude, even for a smaller photon virtuality. We realize that for HERMES kinematics the ratio of interference to squared BH term is not necessary small, which is obviously consistent with the measurement of sizable beam spin and charge asymmetries. However, we have maybe overestimated the ratio (117). If the sea quark contribution is not yet dominant in this fixed target kinematics, we might have an additional suppression by a factor $\sqrt{x_{\mathrm{B}}}$. Indeed, the measurement of the charge asymmetry for a proton target shows that the real part of the interference term is suppressed by a factor of about ten. The unpolarized squared BH amplitude, which can be taken into account exactly, is dominated by its 'constant' term, while higher harmonics are suppressed by the factor $K \sim \sqrt{-(1-y) \Delta^{2} / \mathcal{Q}^{2}}$.

\footnotetext{
${ }^{7}$ This is based on the assumption that the CFF scales like $A^{2} / x_{\mathrm{B}}$ as it follows from Eq. (70) together with the small $x_{\mathrm{B}}$ behavior of the sea quarks. In the valence quark region we expect even a larger suppression.
} 
Since we expect, in the kinematics we are interested in, that the unintegrated asymmetries (112, 113, 114) are dominated by the zero harmonic of the squared BH and first ones of the interference term, the $\phi$ dependence of the $\mathrm{BH}$ propagators will almost cancel each other. Thus, their Fourier series read

$$
\begin{aligned}
A_{\mathrm{LU}}(\phi) & =\sin \left(\phi_{\gamma}^{\prime}\right) A_{\mathrm{LU}}^{(1)}+\cdots, \quad A_{\mathrm{UL}}(\phi)=\sin \left(\phi_{\gamma}^{\prime}\right) A_{\mathrm{UL}}^{(1)}+\cdots, \\
A_{\mathrm{C}}(\phi) & =A_{\mathrm{C}}^{(0)}+\cos \left(\phi_{\gamma}^{\prime}\right) A_{\mathrm{C}}^{(1)}+\cdots,
\end{aligned}
$$

where the Fourier coefficients are proportional to certain linar combinations of twist-two CFFs, contaminated by $1 / \mathcal{Q}^{2}$ power suppressed contributions. For the charge asymmetry $A_{\mathrm{C}}(\phi)$ we included also the 'constant' twist-three contribution, discussed above. To coincide with the definitions, used by the HERMES and CLAS collaborations, we wrote the Fourier expansion with respect to the azimuthal angle of the real photon $\phi_{\gamma}^{\prime}=\pi-\phi$ for a frame in which the $z$-axis points in the direction of the virtual photon momentum. This convention affects only the sign of $A_{\mathrm{C}}^{(1)}$. The asymmetries of $\phi_{\gamma}^{\prime}$ integrated cross sections, e.g.,

$$
A_{\mathrm{LU}}=\frac{2 \int_{0}^{2 \pi} d \phi_{\gamma}^{\prime} \sin \left(\phi_{\gamma}^{\prime}\right)\left(d \sigma^{\uparrow}-d \sigma^{\downarrow}\right)}{\int_{0}^{2 \pi} d \phi_{\gamma}^{\prime}\left(d \sigma^{\uparrow}+d \sigma^{\downarrow}\right)} \approx \frac{2 \int_{0}^{2 \pi} d \phi_{\gamma}^{\prime} \sin ^{2}\left(\phi_{\gamma}^{\prime}\right) / \mathcal{P}_{1} \mathcal{P}_{2}\left(\phi_{\gamma}^{\prime}\right)}{\int_{0}^{2 \pi} d \phi_{\gamma}^{\prime} 1 / \mathcal{P}_{1} \mathcal{P}_{2}\left(\phi_{\gamma}^{\prime}\right)} A_{\mathrm{LU}}^{(1)}
$$

is influenced by the angular dependence of the BH propagators and is generally smaller as the lowest harmonic of the Fourier expansion (118). Moreover, the dependence on the kinematical variables can be altered. Note also that for $x_{\mathrm{B}}, \Delta^{2}$, or $\mathcal{Q}^{2}$ integrated asymmetries, the mean value for different parts of the leptoproduction cross section can be quite different.

\subsection{Analytical approximation in the valence quark region}

The lengthy expressions for the Fourier coefficients make it rather hard to interpret future measurements in terms of GPDs. Thus, it would be instructive to have an approximation at hand that serves as a guide for adjusting model parameters by using efficient numerical codes. This can be done for the observables and kinematics, we discussed above. For instance, the beam spin asymmetry (112) can be simplified to

$$
A_{\mathrm{LU}}(\phi) \sim \pm \frac{x_{\mathrm{A}}}{y} \frac{s_{1, \mathrm{unp}}^{\mathcal{I}}}{c_{0, \text { unp }}^{\mathrm{BH}}} \sin (\phi) \text { with }\left\{\begin{array}{l}
+ \text { for } e^{-} \\
- \text {for } e^{+}
\end{array}\right.
$$

where we neglected possible contamination of the squared DVCS term. Moreover, for $-\Delta_{\text {min }} \ll$ $-\Delta^{2} \ll M_{A}^{2}$ and $x_{\mathrm{B}}=A x_{\mathrm{A}} \lesssim 0.3$ the Fourier coefficients can be drastically simplified due to a rough approximation of kinematical factors, i.e., we expand the Fourier coefficients $c_{0, \text { unp }}^{\mathrm{BH}}$ and $s_{1 \text {,unp }}^{\mathcal{I}}$ (see Appendix $\left[\mathrm{B}\right.$ ) to zero order in $x_{\mathrm{A}}$ and $\tau$. However, we have to pay special attention on terms that contain $G_{3}$ and $\mathcal{H}_{3}$, since $G_{3}(\Delta=0)$ is enhanced by one order of magnitude compared to the normalization of the other two form factors. Thus, we take also $\tau G_{3}$ and $\tau \mathcal{H}_{3}$ into account and obtain for the beam spin asymmetry:

$$
\begin{aligned}
A_{\mathrm{LU}}(\phi) \sim & \pm \frac{x_{\mathrm{A}}(2-y) \sqrt{\frac{-\Delta^{2}(1-y)}{\mathcal{Q}^{2}}}}{2-2 y+y^{2}} \\
& \times \Im \mathrm{m} \frac{2 G_{1} \mathcal{H}_{1}+\left(G_{1}-2 \tau G_{3}\right)\left(\mathcal{H}_{1}-2 \tau \mathcal{H}_{3}\right)+\frac{2}{3} \tau G_{3} \mathcal{H}_{5}}{2 G_{1}^{2}+\left(G_{1}-2 \tau G_{3}\right)^{2}} \sin (\phi) .
\end{aligned}
$$


To demonstrate that these considerations are more on a rough quantitative level, we mention that the kinematical prefactor $1 \leq(2-y) /\left(2-2 y+y^{2}\right) \leq(1+\sqrt{2}) / 2$ should for consistency also be neglected, which can induce a reduction of $21 \%$. In the following we take it into account. One realizes that the single beam spin asymmetry is proportional to a linear combination of nucleus GPDs multiplied with $x_{\mathrm{A}}=x_{\mathrm{B}} / A$. Since for large $A$ the form factors scale with $Z$ and the CFFs with $A^{2}$, this asymmetry does not scale with $A$, however, besides bound state effects it only depends on the ratio $A / Z$. It is rather surprising that bound state effects enter already the interference term for the unpolarized target not only due to a possible modification of the scaling relation for $\mathcal{H}_{1}$, but also directly by the appearance of $\mathcal{H}_{3}$ and $\mathcal{H}_{5}$.

In the case that the beam spin asymmetry is measured on an incomplete unpolarized target, where only over the polarization states with $\Lambda= \pm 1$ is summed, the prediction differs from the asymmetry (121):

$$
A_{\mathrm{L} \pm}(\phi) \sim \pm \frac{x_{\mathrm{A}}}{y} \frac{s_{1, \mathrm{unp}}^{\mathcal{I}}-\frac{1}{3} s_{1, \mathrm{LLP}}^{\mathcal{I}}}{c_{0, \mathrm{unp}}^{\mathrm{BH}}-\frac{1}{3} c_{0, \mathrm{LLP}}^{\mathrm{BH}}} \sin (\phi) \quad \text { with } \quad\left\{\begin{array}{l}
+ \text { for } e^{-} \\
- \text {for } e^{+}
\end{array}\right.
$$

and reads in our kinematical approximation

$$
\begin{aligned}
A_{\mathrm{L} \pm}(\phi) \sim & \pm \frac{x_{\mathrm{A}}(2-y) \sqrt{\frac{-\Delta^{2}(1-y)}{\mathcal{Q}^{2}}}}{2-2 y+y^{2}} \\
& \times \Im \mathrm{m} \frac{2 G_{1}\left(\mathcal{H}_{1}-\frac{1}{3} \mathcal{H}_{5}\right)+2\left(G_{1}-2 \tau G_{3}\right)\left(\mathcal{H}_{1}-2 \tau \mathcal{H}_{3}-\frac{1}{3} \mathcal{H}_{5}\right)}{2 G_{1}^{2}+2\left(G_{1}-2 \tau G_{3}\right)^{2}} \sin (\phi) .
\end{aligned}
$$

The approximation of the unpolarized charge asymmetry (114)

$$
A_{\mathrm{C}}(\phi) \sim \frac{x_{\mathrm{A}}}{y} \frac{c_{0, \text { unp }}^{\mathcal{I}}+c_{1, \text { unp }}^{\mathcal{I}} \cos (\phi)}{c_{0, \text { unp }}^{\mathrm{BH}}}+\cdots
$$

is obtained in the analogous manner. The dominant twist-two harmonic is given as the real part of the same linear combination of GPDs as in the beam spin asymmetry (121):

$$
\begin{aligned}
A_{\mathrm{C}}(\phi) \sim & \frac{x_{\mathrm{A}} \sqrt{\frac{-\Delta^{2}}{\mathcal{Q}^{2}}(1-y)}}{y} \\
& \times \Re \mathrm{e} \frac{2 G_{1} \mathcal{H}_{1}+\left(G_{1}-2 \tau G_{3}\right)\left(\mathcal{H}_{1}-2 \tau \mathcal{H}_{3}\right)+\frac{2}{3} \tau G_{3} \mathcal{H}_{5}}{2 G_{1}^{2}+\left(G_{1}-2 \tau G_{3}\right)^{2}} \cos (\phi)+\cdots .
\end{aligned}
$$

Also this asymmetry is nearly independent on $A$. As noted before, the constant term only depends on twist-two GPDs and does not vanish at the kinematical boundaries. In our approximation it is

$$
\frac{c_{0, \text { unp }}^{\mathcal{I}}}{c_{1, \text { unp }}^{\mathcal{I}}} \simeq \frac{2-y}{\sqrt{1-y}} \sqrt{\frac{-\Delta^{2}}{\mathcal{Q}^{2}}} .
$$

For a scalar target the exact relation does not depend on GPDs [6], while for a spin-1/2 target only a $x_{\mathrm{A}}$ suppressed dependence appears [53, 7]. For a spin-1 target the analogous relation is unknown at present. 
The single spin asymmetry (113) for longitudinally polarized target,

$$
A_{\mathrm{UL}}(\phi) \simeq \pm \frac{x_{\mathrm{A}}}{y} \frac{s_{1, \mathrm{LP}}^{\mathcal{I}}}{c_{0, \text { unp }}^{\mathrm{BH}}} \sin (\phi)+\cdots \quad \text { with } \quad\left\{\begin{array}{l}
+ \text { for } e^{-} \\
- \text {for } e^{+}
\end{array},\right.
$$

is mainly governed by $\widetilde{\mathcal{H}}_{1}$ :

$$
A_{\mathrm{UL}}(\phi) \sim \pm \frac{x_{\mathrm{A}} \sqrt{\frac{-\Delta^{2}}{\mathcal{Q}^{2}}(1-y)}}{y} \Im \mathrm{m} \frac{3\left(G_{1}-\tau G_{3}\right) \widetilde{\mathcal{H}}_{1}+\frac{3 x_{\mathrm{A}}}{2} G_{2}\left(\mathcal{H}_{1}-\tau \mathcal{H}_{3}-\mathcal{H}_{5}\right)}{2 G_{1}^{2}+\left(G_{1}-2 \tau G_{3}\right)^{2}} \sin (\phi) .
$$

Since the polarized quark contribution is relatively small compared to the unpolarized ones and the latter increase faster with growing $1 / x_{\mathrm{B}}$ (in the small $x_{\mathrm{B}}$ region), we included also a $x_{\mathrm{A}}$ suppressed term that is proportional to $\mathcal{H}_{1}-\tau \mathcal{H}_{3}-\mathcal{H}_{5}$.

For a spin-1 target another quite interesting observable is the tensor polarization asymmetry (116). Their measurement requires a longitudinally polarized target. For an unpolarized beam this asymmetry is dominated by the squared $\mathrm{BH}$ contribution, which provides a dominant constant term that is proportional to $\tau G_{3}$ :

$$
\begin{aligned}
A_{z z}(\phi)=\frac{-4 \tau G_{3}\left(G_{1}-\tau G_{3}\right)}{2 G_{1}^{2}+\left(G_{1}-2 \tau G_{3}\right)^{2}} \pm \frac{x_{\mathrm{A}} \sqrt{\frac{-\Delta^{2}}{\mathcal{Q}^{2}}(1-y)}}{y} \\
\quad \times 3 G_{1}\left[\cos (\phi) \times \ldots+\lambda \frac{y(2-y)}{2-2 y+y^{2}} \sin (\phi) \times \ldots\right]+\cdots .
\end{aligned}
$$

Here the ellipsises, proportional to the first harmonics, arise from a Taylor expansion with respect to $-\Delta^{2} / \mathcal{Q}^{2}$ and stand for the real and imaginary part of a certain linear combination of CFFs:

$$
\mathcal{H}_{1}+\frac{2}{3} \mathcal{H}_{5}-\frac{G_{1}}{2 G_{1}^{2}+\left(G_{1}-2 \tau G_{3}\right)^{2}}\left(2 G_{1} \mathcal{H}_{1}+\left(G_{1}-2 \tau G_{3}\right)\left(\mathcal{H}_{1}-2 \tau \mathcal{H}_{3}\right)+\frac{2 \tau}{3} G_{3} \mathcal{H}_{5}\right) .
$$

The third term comes from the denominator, while the $\cos (\phi)$ term that stems from the BH Fourier coefficients $c_{1, \text { unp }}^{\mathrm{BH}}$ and $c_{1, \mathrm{LLP}}^{\mathrm{BH}}$ do not contribute in our approximation. Consequently, to extract the interference term the squared $\mathrm{BH}$ contribution has to be subtracted. Alternatively, we can form the tensor asymmetry from the charge odd part of the cross section

$$
A_{\mathrm{C} z z}(\phi)=\frac{d \sigma_{z z}^{e^{+}}-d \sigma_{z z}^{e^{-}}}{3 d \sigma_{\mathrm{unp}}^{e^{+}}+3 d \sigma_{\mathrm{unp}}^{e^{-}}} \simeq-\frac{x_{\mathrm{A}}}{y} \frac{c_{1, \mathrm{unp}}^{\mathcal{I}}-c_{1, \mathrm{LLP}}^{\mathcal{I}}}{c_{0, \mathrm{unp}}^{\mathrm{BH}}} \cos (\phi)+\cdots
$$

where the ellipsis include a constant term and higher harmonics. Another possibility is to use a polarized beam and to form the asymmetry

$$
\begin{aligned}
A_{\mathrm{L} z z}(\phi) & =\frac{d \sigma_{z z}(\lambda=+1)-d \sigma_{z z}(\lambda=-1)}{3 d \sigma_{\mathrm{unp}}(\lambda=+1)+3 d \sigma_{\mathrm{unp}}(\lambda=-1)} \\
& \simeq \pm \frac{x_{\mathrm{A}}}{y} \frac{s_{1, \mathrm{unp}}^{\mathcal{I}}-s_{1, \mathrm{LLP}}^{\mathcal{I}}}{c_{0, \mathrm{unp}}^{\mathrm{BH}}} \sin (\phi)+\cdots \quad \text { with }\left\{\begin{array}{l}
+ \text { for } e^{-} \\
- \text {for } e^{+}
\end{array}\right.
\end{aligned}
$$


which is dominated by the $\sin (\phi)$ harmonics of the interference term only. These two asymmetries allow to explore the real and imaginary part of the following linear combination of GPDs:

$$
\begin{aligned}
\left\{\begin{array}{l}
A_{\mathrm{L} z z} \\
A_{\mathrm{C} z z}
\end{array}\right\}(\phi) \sim & \sqrt{\frac{-\Delta^{2}}{\mathcal{Q}^{2}}(1-y)} 2 x_{\mathrm{A}}\left\{\begin{array}{c}
\mp 1 \\
-1 / y
\end{array}\right\}\left\{\begin{array}{c}
\sin (\phi) \\
\cos (\phi)
\end{array}\right\} \\
& \times\left\{\begin{array}{c}
\Im \mathrm{m} \\
\Re \mathrm{e}
\end{array}\right\} \frac{G_{1}\left(\tau \mathcal{H}_{3}+\mathcal{H}_{5}\right)+\tau G_{3}\left(\mathcal{H}_{1}-2 \tau \mathcal{H}_{3}-\frac{1}{3} \mathcal{H}_{5}\right)}{2 G_{1}^{2}+\left(G_{1}-2 \tau G_{3}\right)^{2}}
\end{aligned}
$$

It is now instructive to compare these results with those for a spin- $1 / 2$ or -0 target and to explore its dependence on GPDs, which are of course model dependent. The analogous predictions simply follow by setting $G_{3}, \mathcal{H}_{3}$, and $\mathcal{H}_{5}$ to zero and by the replacements:

$$
G_{1} \rightarrow\left\{\begin{array}{c}
F_{1} \\
F
\end{array}\right\}, G_{2} \rightarrow\left\{\begin{array}{c}
F_{1}+F_{2} \\
0
\end{array}\right\}, \mathcal{H}_{1} \rightarrow\left\{\begin{array}{l}
\mathcal{H} \\
\mathcal{H}
\end{array}\right\}, \widetilde{\mathcal{H}}_{1} \rightarrow\left\{\begin{array}{c}
\widetilde{\mathcal{H}} \\
0
\end{array}\right\} \quad \text { for spin- }\left\{\begin{array}{c}
1 / 2 \\
0
\end{array}\right.
$$

First we like to remind that the sum rules, e.g., Eq. (94), suggest that the $\Delta^{2}$-dependence of the valence-like GPDs is given by $G_{i}$ and $F_{1}$ (or the scalar form factor $F$ ), respectively. Furthermore, the analyses of the H1, HERMES and CLAS DVCS data for a proton target in terms of the oversimplified model, given in Section 4, at LO indicate that the unpolarized sea quark contribution is compared to the forward case additionally suppressed ${ }^{8}$. We suppose that this qualitative property holds also true for the nucleus GPDs. Therefore, we neglect in the valence region the sea quark contribution and model the remaining GPDs as a product of form factor and valence quark distributions ${ }^{9}$. In LO of perturbation theory we find for nuclei targets

$$
\begin{aligned}
\frac{x_{\mathrm{A}} \Im \mathrm{m} \mathcal{H}^{A}\left(\xi, \Delta^{2}\right)}{F^{A}\left(\Delta^{2}\right)} & \sim \pi x_{\mathrm{A}}\left\{Q_{u}^{2} q^{u_{\text {val }} / \mathrm{A}}(\xi)+Q_{d}^{2} q^{d_{\text {val }} / \mathrm{A}}(\xi)\right\} \\
& \sim \pi \frac{Z Q_{u}^{2}+N Q_{d}^{2}}{Z} x_{\mathrm{B}}\left\{q^{u_{\text {val }}}\left(\frac{x_{\mathrm{B}}}{2-x_{\mathrm{B}}}\right)+q^{d_{\text {val }}}\left(\frac{x_{\mathrm{B}}}{2-x_{\mathrm{B}}}\right)\right\}
\end{aligned}
$$

expressed in terms of the quark densities of the proton. The analogous formula holds true for the spin- $1 / 2$ case and also for the $\mathcal{H}_{1}$ contribution of spin- 1 targets.

The beam spin asymmetry for the positron scattering off a proton target is

$$
A_{\mathrm{LU}}(\phi) \sim-\left.\sqrt{\frac{-\Delta^{2}(1-y)}{\mathcal{Q}^{2}}} \pi x_{\mathrm{B}}\left\{Q_{u}^{2} q^{u_{\mathrm{val}}}\left(\xi_{N}\right)+Q_{d}^{2} q^{d_{\mathrm{val}}}\left(\xi_{N}\right)\right\} \sin (\phi)\right|_{\xi_{N}=\frac{x_{\mathrm{B}}}{2-x_{\mathrm{B}}}}
$$

The ratio of beam spin asymmetries for a spin- 0 or $-1 / 2$ nucleus with $Z \simeq N$ to the proton is

$$
\left.\frac{A_{\mathrm{LU}}^{\mathrm{A}}(\phi)}{A_{\mathrm{LU}}(\phi)} \sim \frac{\left(Q_{u}^{2}+Q_{d}^{2}\right)\left\{q^{u_{\mathrm{val}}}(\xi)+q^{d_{\mathrm{val}}}\left(\xi_{N}\right)\right\}}{Q_{u}^{2} q^{u_{\mathrm{val}}}\left(\xi_{N}\right)+Q_{d}^{2} q^{d_{\mathrm{val}}}\left(\xi_{N}\right)}\right|_{\xi_{N}=\frac{x_{\mathrm{B}}}{2-x_{\mathrm{B}}}} \quad \text { for } \quad J=\{0,1 / 2\} .
$$

\footnotetext{
${ }^{8}$ We remind that $\xi \approx x_{\mathrm{B}} /\left(2-x_{\mathrm{B}}\right)$ and already for $\xi \sim 0.05$ one finds in deep inelastic scattering an almost equal contribution of valence and sea quarks.

${ }^{9}$ Here we neglect any skewedness effect. For the DD model, introduced in Section 4 skewedness effects would provide an enhancement of the CFFs. Strictly spoken, it is not known what caused the observed suppression of sea quark contributions and thus the simple ansatz for the DD's together with a rather large slope parameter might be questionable.
} 
For a spin-1 nucleus we would have the same prediction, if $\mathcal{H}_{3}^{\text {val }}=G_{3} / G_{1} \mathcal{H}_{1}^{\text {val }}$ holds true. However, if $\mathcal{H}_{3}^{\text {val }}$ is negligible in this kinematics, it follows from Eq. (121) an additional $\Delta^{2}$-dependence, which is crucial:

$$
\begin{aligned}
& \left.\frac{A_{\mathrm{LU}}^{\mathrm{A}}(\phi)}{A_{\mathrm{LU}}(\phi)} \sim \frac{3-2 \tau G_{3} / G_{1}}{2+\left(1-2 \tau G_{3} / G_{1}\right)^{2}} \frac{\left(Q_{u}^{2}+Q_{d}^{2}\right)\left\{q^{u_{\mathrm{val}}}\left(\xi_{N}\right)+q^{d_{\mathrm{val}}}\left(\xi_{N}\right)\right\}}{Q_{u}^{2} q^{u_{\mathrm{val}}}\left(\xi_{N}\right)+Q_{d}^{2} q^{d_{\mathrm{val}}}\left(\xi_{N}\right)}\right|_{\xi_{N}=\frac{x_{\mathrm{B}}}{2-x_{\mathrm{B}}}} \text { for } \quad J=1, \\
& \left.\frac{A_{\mathrm{L} \pm}^{\mathrm{A}}(\phi)}{A_{\mathrm{LU}}(\phi)} \sim \frac{3-3 \tau G_{3} / G_{1}}{2+2\left(1-\tau G_{3} / G_{1}\right)^{2}} \frac{\left(Q_{u}^{2}+Q_{d}^{2}\right)\left\{q^{u_{\mathrm{val}}}\left(\xi_{N}\right)+q^{d_{\mathrm{val}}}\left(\xi_{N}\right)\right\}}{Q_{u}^{2} q^{u_{\mathrm{val}}}\left(\xi_{N}\right)+Q_{d}^{2} q^{d_{\mathrm{val}}}(\xi)}\right|_{\xi_{N}=\frac{x_{\mathrm{B}}}{2-x_{\mathrm{B}}}} .
\end{aligned}
$$

Taking $x_{\mathrm{B}} \sim 0.1$, we expect from the parameterization of parton densities that $d_{\mathrm{val}} / u_{\mathrm{val}} \sim 0.5$. For $\Delta^{2}=-0.2 \mathrm{GeV}^{2}, \mathcal{Q}^{2}=2.5 \mathrm{GeV}^{2}$, and $E=27.5 \mathrm{GeV}$ we estimate the beam spin asymmetry for a proton target and the ratios (135) and (136) for nuclei $(Z \simeq N)$ to be:

$$
A_{\mathrm{LU}}(\phi) \sim-0.29 \sin (\phi), \quad \frac{A_{\mathrm{LU}}^{\mathrm{A}}(\phi)}{A_{\mathrm{LU}}(\phi)} \sim\left\{\begin{array}{c}
5 / 3 \\
1
\end{array}\right\} \text { for } J=\left\{\begin{array}{c}
\{0,1 / 2\} \\
1
\end{array}\right\} .
$$

Our estimate for the $\sin (\phi)$-weighted asymmetry (119) is $A_{\mathrm{LU}} \sim-0.23$, while the ratios of beam spin asymmetries for different targets are unchanged. So we roughly expect an enhancement of the beam spin asymmetry for nuclei with spin-0 and $-1 / 2$, which simply arises from the ratio of squared charges for an isoscalar to an isodoublet state and the $u$ quark dominance. For a spin-1 target, one would expect the same. However, in the case that $\mathcal{H}_{3}$ (and also $\mathcal{H}_{5}$ ) does not contribute the factor $\left(3-2 \tau G_{3} / G_{1}\right) /\left(2+\left(1-2 \tau G_{3} / G_{1}\right)^{2}\right)$ gives a $\Delta^{2}$ dependent suppression. It decreases with growing $\left|\Delta^{2}\right|<0.5 \mathrm{GeV}^{2}$, reaches its minimum at $\left|\Delta^{2}\right| \approx 0.5 \mathrm{GeV}^{2}$ with $\approx-0.1$ and then increases with growing $\left|\Delta^{2}\right|$. Note the sign change of the beam spin asymmetry, which occurs in the region $0.4 \mathrm{GeV}^{2}<-\Delta^{2}<0.7 \mathrm{GeV}^{2}$.

In the following we confront our oversimplified estimates for the $\phi$-integrated beam spin asymmetry (119) with preliminary HERMES data from the 2000 run, which have been taken for proton, deuteron, and neon target [27]:

$$
\begin{aligned}
A_{\mathrm{LU}} & =-0.18 \pm 0.03 \pm 0.03,\left\langle x_{\mathrm{B}}\right\rangle=0.12,\left\langle-\Delta^{2}\right\rangle=0.18 \mathrm{GeV}^{2},\left\langle\mathcal{Q}^{2}\right\rangle=2.5 \mathrm{GeV}^{2} \\
A_{\mathrm{LU}}^{\mathrm{Ne}} & =-0.22 \pm 0.03 \pm 0.03,\left\langle x_{\mathrm{B}}\right\rangle=0.09,\left\langle-\Delta^{2}\right\rangle=0.13 \mathrm{GeV}^{2},\left\langle\mathcal{Q}^{2}\right\rangle=2.2 \mathrm{GeV}^{2} \\
A_{\mathrm{L} \pm \mathrm{U}}^{d} & =-0.15 \pm 0.03 \pm 0.03,\left\langle x_{\mathrm{B}}\right\rangle=0.1,\left\langle-\Delta^{2}\right\rangle=0.2 \mathrm{GeV}^{2},\left\langle\mathcal{Q}^{2}\right\rangle=2.5 \mathrm{GeV}^{2},
\end{aligned}
$$

where the first (second) error denotes the statistical (systematical) uncertainty. Here the notation $A_{\mathrm{L} \pm \mathrm{U}}^{d}$ refers to the fact that this value is obtained from two different data sets of polarized and unpolarized deuteron target. Our rather naive estimates give

$$
A_{\mathrm{LU}}=-0.26, \quad A_{\mathrm{LU}}^{\mathrm{Ne}}=-0.34, \quad A_{\mathrm{LU}}^{d}=-0.23, A_{\mathrm{L} \pm}^{d}=-0.21,
$$

which are slightly higher than the experimental values. Note that these numbers include uncertainties induced by the kinematical approximation and, of course, due to the simplification of GPDs. Compared to the beam spin asymmetry on a proton target from the 1996/97 run

$$
A_{\mathrm{LU}}=-0.23 \pm 0.04 \pm 0.03,\left\langle x_{\mathrm{B}}\right\rangle=0.11,\left\langle-\Delta^{2}\right\rangle=0.27 \mathrm{GeV}^{2},\left\langle\mathcal{Q}^{2}\right\rangle=2.6 \mathrm{GeV}^{2}
$$

our naive analytical and numerical estimates, within a certain model that contains also sea quarks and twist-three corrections, give in both cases nearly the same value $A_{\mathrm{LU}}=-0.26$ and 
$A_{\mathrm{LU}}=-0.27$, respectively. The sea quark content gives an enhancement and the exact kinematics at twist-tree level a dumping, which results finally in a similar prediction. Nevertheless, these numbers are rather model dependent and in fact by varying the slope $B_{\text {sea }}, b$-parameters, and adding dynamical twist-tree contributions by 'hand' our prediction covered the range $0.16 \leq\left|A_{\mathrm{LU}}\right| \leq 0.37$ 7.

For JLAB kinematics with $x_{\mathrm{B}}=0.3[0.2], E=6[12] \mathrm{GeV}, \Delta^{2}=-0.25 \mathrm{GeV}^{2}$, and $\mathcal{Q}^{2}=$ $2.5 \mathrm{GeV}^{2}$ the quantitative estimates for proton, deuteron, and a isoscalar nuclei $A$ target are

$$
A_{\mathrm{LU}} \sim 0.25[0.3], \quad A_{\mathrm{LU}}^{d} \sim 0.2[0.25], \quad A_{\mathrm{LU}}^{\mathrm{A}} \sim 0.35[0.45] .
$$

For the charge asymmetry, there are important differences with respect to the beam spin asymmetry. First we recall the non negligible constant term. Moreover, this asymmetry arises from the real part of the Compton amplitude and so it is also sensitive to the $D$-term, which drops out in the imaginary part ${ }^{10}$. At smaller values of $x_{\mathrm{B}}$ the ratio of real to imaginary part is

$$
\mathcal{R}=\frac{\Re \mathrm{e} \mathcal{F}}{\Im \mathrm{m} \mathcal{F}} \simeq \tan \left(\left[2 \alpha^{i}-1-\mathcal{S}_{\mathcal{F}}\right] \frac{\pi}{4}\right)+\text { pure 'exclusive' contributions } / \Im \mathrm{m} \mathcal{F},
$$

where the signature $\mathcal{S}_{\mathcal{F}}=1$ for the $\operatorname{CFFs}\left\{\mathcal{H}, \mathcal{E}, \mathcal{H}_{1}, \ldots, \mathcal{H}_{5}\right\}$ and $\mathcal{S}_{\mathcal{F}}=-1$ for the set $\left\{\widetilde{\mathcal{H}}, \widetilde{\mathcal{E}}, \widetilde{\mathcal{H}}_{1}, \ldots, \widetilde{\mathcal{H}}_{4}\right\}$. Here we used that the GPDs behave like $H(\xi) \propto \xi^{-\alpha^{i}}$ with $\alpha^{i}>0$. Since $\alpha^{i} \sim 1 / 2$ and $\alpha^{i}>1$ for unpolarized valence and sea quarks, respectively, we expect also for larger values of $x_{\mathrm{B}}$ that the valence and sea quarks give a negative and positive correction, respectively:

$$
R^{i}=\frac{\Re \mathrm{e} \mathcal{H}^{i}}{\Im \mathrm{m} \mathcal{H}^{i}}=\left\{\begin{array}{c}
<0 \\
>0
\end{array}\right\} \quad \text { for } \quad\left\{\begin{array}{c}
\text { valence quarks } \\
\text { sea quarks }
\end{array} .\right.
$$

The $D$-term contribution has been estimated to be negative, too, and will partially cancel the positive sea quark contribution. All these terms enter the Fourier coefficient $A_{\mathrm{C}}^{(1)}$, defined in Eq. (118) with a reversed sign. The HERMES data

$$
A_{\mathrm{C}}(\phi)=-0.05 \pm 0.03(\text { stat })+[0.11 \pm 0.04(\text { stat })] \cos \left(\phi_{\gamma}^{\prime}\right)
$$

for $\left\langle x_{\mathrm{B}}\right\rangle=0.12,\left\langle-\Delta^{2}\right\rangle=0.27 \mathrm{GeV}^{2},\left\langle\mathcal{Q}^{2}\right\rangle=2.7 \mathrm{GeV}^{2}$, can be explained by two different scenarios:

1. small sea quark contribution and rather small or no $D$-term

2. large sea quark contribution and large negative $D$-term.

The second scenario predicts a beam spin asymmetry of $A_{\mathrm{LU}} \simeq-0.37$, which is slightly larger as the beam spin asymmetry of the 1996/97 run. So a definite conclusion about the $D$-term can not be done with present data. However, we state: if high precision data will be available, the ratio of charge asymmetry to beam spin asymmetry allows to pin down the $D$-term contribution. The measured ratio

$$
\frac{A_{\mathrm{C}}^{(0)}}{A_{\mathrm{C}}^{(1)}}=-\frac{c_{0, \text { unp }}^{\mathcal{I}}}{c_{1, \text { unp }}^{\mathcal{I}}}-\sim 0.5
$$

\footnotetext{
${ }^{10} \mathrm{~A}$ first discussion of the imaginary and real part of the Compton amplitude in the context of field theory can be found for instance in Ref. [59, 60. The constant term in the real part, which appears here due to the $D$-term, has been related to a fixed pole in the language of Reggeization.
} 
coincides with our estimate $(125)$, which yields the value $\approx 0.65$.

In both scenarios one expects that the valence quark contribution dominates in the real part of the $\mathrm{CFF} \mathcal{H}$ for HERMES kinematics. The real part of this dominant CFF for a proton and a scalar nuclei target are

$$
\begin{aligned}
\frac{x_{\mathrm{B}} \Re \mathrm{e} \mathcal{H}_{1}\left(\xi, \Delta^{2}\right)}{F_{1}\left(\Delta^{2}\right)} \sim \pi x_{\mathrm{B}}\left\{Q_{u}^{2} R^{u_{\text {val }}} q^{u_{\text {val }}}+Q_{d}^{2} R^{d_{\text {val }}} q^{u_{\text {val }}} \sum_{i} Q_{i}^{2} \bar{q}^{i}\right\}, \\
\frac{x_{\mathrm{A}} \Re \mathrm{e} \mathcal{H}^{\mathrm{A}}\left(\xi, \Delta^{2}\right)}{F^{\mathrm{A}}\left(\Delta^{2}\right)} \sim \pi x_{\mathrm{B}}\left\{\frac{Z Q_{u}^{2}+N Q_{d}^{2}}{Z}\left[R^{u_{\text {val }}} q^{u_{\text {val }}}+R^{d_{\text {val }}} q^{u_{\text {val }}}\right]\right\},
\end{aligned}
$$

where $R^{i}$ and $q^{i}$ depend on $\xi_{N}=x_{\mathrm{B}} /\left(2-x_{\mathrm{B}}\right), \Delta^{2}$, and $\mathcal{Q}^{2}$. As above in the case of the imaginary part a similar estimate holds true for a spin- $1 / 2$ nucleus target. For larger value of $x_{\mathrm{B}}$, e.g. $x_{\mathrm{B}}=0.12$ the asymptotic formula (142) overestimates the size of the real part. In the MRS A' parameterization we find $R^{u_{\mathrm{val}}}\left(\xi_{N}=0.065\right) \sim-0.4$ and $R^{d_{\mathrm{val}}}\left(\xi_{N}=0.065\right) \sim-0.25$. So we expect, e.g., $\mathcal{Q}^{2}=2.7 \mathrm{GeV}^{2}$ the following ratio of charge and single beam asymmetries ${ }^{11}$ on a proton target

$$
\frac{A_{\mathrm{C}}^{(1)}}{A_{\mathrm{LU}}^{(1)}} \sim \frac{2-2 y+y^{2}}{(2-y) y} \frac{Q_{u}^{2} R^{u_{\mathrm{val}}} q^{u_{\mathrm{val}}}+Q_{d}^{2} R^{d_{\mathrm{val}}} q^{d_{\mathrm{val}}}}{Q_{u}^{2} q^{u_{\mathrm{val}}}+Q_{d}^{2} q^{d_{\mathrm{val}}}} \sim-0.8
$$

which is consistent with HERMES data. For an isoscalar nucleus we expect a similar value:

$$
\frac{A_{\mathrm{C}}^{\mathrm{A}(1)}}{A_{\mathrm{LU}}^{\mathrm{A}(1)}} \sim \frac{2-2 y+y^{2}}{(2-y) y} \frac{R^{u_{\mathrm{val}}} q^{u_{\mathrm{val}}}+R^{d_{\mathrm{val}}} q^{d_{\mathrm{val}}} \sum_{i} Q_{i}^{2} \bar{q}^{i}}{q^{u_{\mathrm{val}}}+q^{d_{\mathrm{val}}}} \sim-0.7 .
$$

However, these ratios should be smaller in reality, since the sea quarks enter the numerator and denominator with negative and positive sign, respectively.

The ratio of charge to beam spin asymmetry for a spin-1 target has a rather complex model dependence. Even if we set $\mathcal{H}_{3}^{\text {val }}$ and $\mathcal{H}_{5}$ to zero, we should worry about the unknown 'quadrupole' contribution $\mathcal{H}_{3}$, induced by the sea quarks:

$$
\frac{A_{\mathrm{C}}^{\mathrm{A}(1)}}{A_{\mathrm{LU}}^{\mathrm{A}(1)}} \sim \frac{2-2 y+y^{2}}{(2-y) y} \frac{R^{u_{\text {val }}} q^{u_{\text {val }}}+R^{d_{\text {val }}} q^{d_{\text {val }}}+2 \frac{G_{1}^{\text {sea }}}{G_{1}}\left[1-\frac{2 \tau G_{3}^{\text {sea }}\left(G_{1}-2 \tau G_{3}\right)}{G_{1}^{\text {sea }}\left(3 G_{1}-2 \tau G_{3}\right)}\right] R^{\text {sea }} \frac{2 \sum_{i} Q_{i}^{2} \bar{q}^{i}}{\sum_{i=u, d} Q_{i}^{2}}}{q^{u_{\text {val }}}+q^{d_{\text {val }}}+2 \frac{G_{1}^{\text {sea }}}{G_{1}}\left[1-\frac{2 \tau G_{\mathrm{sea}}^{\text {sea }}\left(G_{1}-2 \tau G_{3}\right)}{G_{1}^{\text {sea }}\left(3 G_{1}-2 \tau G_{3}\right)}\right] \frac{2 \sum_{i} Q_{i}^{2} \bar{q}^{i}}{\sum_{i=u, d} Q_{i}^{2}}} .
$$

Here we have set for simplicity $\mathcal{H}_{3}^{\text {sea }} / \mathcal{H}_{1}^{\text {sea }}=G_{3}^{\text {sea }} / G_{1}^{\text {sea }}=1-\mu_{d}^{\text {sea }}+\mathcal{Q}_{d}^{\text {sea }}$ and assumed the same $R^{\text {sea }}$ for all sea quark species. If the sea quark contributions are small, we would have the same ratio (148) as for isoscalar nucleus with spin-0 or $-1 / 2$.

It is interesting that for larger values of $x_{\mathrm{B}}$ the sign of the real part of the CFFs might be changed. In our model that happens for valence $u[d]$ quarks at $x_{\mathrm{B}} \sim 0.3[0.2]$, while the sign of sea quarks and $D$-term remains the same. There is also a significant difference between these contributions. While the real part of the sea quarks is expected to drop rather fast with increasing

\footnotetext{
${ }^{11}$ This ratio has the advantage that the $\mathrm{BH}$ contribution and partly the kinematical prefactors of the interference term drop out and so it is after restoration of the $y$ dependence exact up to the neglected squared DVCS term and the approximation of the CFFs. $\widetilde{H}$ and $E$ CFFs, which are kinematically suppressed, are still neglected.
} 
$x_{\mathrm{B}}$, the $D$-term gives a constant contribution. JLAB kinematics would be suitable to explore this region and provide so more insight in the structure of GPDs, unfortunately, no positron beam is available.

Now we come to the longitudinally polarized target spin asymmetry. For a proton target the ratio of this target spin to the beam spin asymmetry is

$$
\frac{A_{\mathrm{UL}}(\phi)}{\mathrm{A}_{\mathrm{LU}}(\phi)} \sim \frac{2-2 y+y^{2}}{(2-y) y}\left[\frac{Q_{u}^{2} \Delta q^{u_{\mathrm{val}}}+Q_{d}^{2} \Delta q^{d_{\mathrm{val}}}}{Q_{u}^{2} q^{u_{\mathrm{val}}}+Q_{d}^{2} q^{d_{\mathrm{val}}}}+\frac{x_{\mathrm{B}}}{2} \frac{F_{2}}{F_{1}}\right] .
$$

Both terms in the squared bracket on the r.h.s. are positive and become smaller ${ }^{12}$ with decreasing $x_{\mathrm{B}}$. So within our simplifications we expect the same sign as for the beam spin asymmetry and a rather small value for not so large $x_{\mathrm{B}}$ or small $y$. In the case of a isoscalar nuclei spin- $1 / 2$ target

$$
\frac{A_{\mathrm{UL}}^{\mathrm{A}}(\phi)}{A_{\mathrm{LU}}^{\mathrm{A}}(\phi)} \sim \frac{2-2 y+y^{2}}{(2-y) y}\left[\frac{\Delta q^{u_{\mathrm{val}}}+\Delta q^{d_{\mathrm{val}}}}{q^{u_{\mathrm{val}}}+q^{d_{\mathrm{val}}}}+\frac{x_{\mathrm{B}}}{2 A} \frac{F_{2}^{\mathrm{A}}}{F_{1}^{\mathrm{A}}}\right]
$$

the form factor dependent part will be suppressed by the atomic mass number $A$. As long as this term is not enhanced due to a node in $F_{1}^{\mathrm{A}}\left(\Delta_{0}^{2}\right)$ at given $\Delta_{0}^{2}$, the ratio of polarized to unpolarized quark distribution dominates and we expect for $x_{\mathrm{B}} \approx 0.2, \mathcal{Q}^{2} \approx 2 \mathrm{GeV}^{2}$ and HERMES [JLAB@12 $\mathrm{GeV}]$ kinematics

$$
\frac{A_{\mathrm{UL}}^{\mathrm{A}}(\phi)}{A_{\mathrm{LU}}^{\mathrm{A}}(\phi)} \sim \frac{2-2 y+y^{2}}{(2-y) y} \frac{\Delta q^{u_{\mathrm{val}}}+\Delta q^{d_{\mathrm{val}}}}{q^{u_{\mathrm{val}}}+q^{d_{\mathrm{val}}}} \sim 0.5[0.2]
$$

If we neglect $\mathcal{H}_{3}$ and $\mathcal{H}_{5} \mathrm{CFF}$, we find for an isoscalar spin-1 target a similar prediction:

$$
\frac{A_{\mathrm{UL}}^{\mathrm{A}}(\phi)}{A_{\mathrm{LU}}^{\mathrm{A}}(\phi)} \sim \frac{2-2 y+y^{2}}{(2-y) y} \frac{G_{1}-\tau G_{3}}{G_{1}-2 \tau G_{3} / 3}\left[\frac{\Delta q^{u_{\mathrm{val}}}+\Delta q^{d_{\mathrm{val}}}}{q^{u_{\mathrm{val}}}+q^{d_{\mathrm{val}}}}+\frac{x_{\mathrm{B}}}{2 A} \frac{G_{2}}{G_{1}-\tau G_{3}}\right],
$$

which differs from the previous cases by a $\Delta^{2}$ dependent prefactor. Unfortunately, this ratio is also rather sensitive to the details of $H_{3}$ and $H_{5}$ GPDs, compare Eqs. (121) and (127), and so no definite estimate can be given.

Finally, we discuss the tensor polarization asymmetries. In the case that $\mathcal{H}_{3}$ and $\mathcal{H}_{5}$, are small we find that these asymmetries can be expressed by the charge and beam spin asymmetries:

$$
\frac{A_{\mathrm{L} z z}^{\mathrm{A}}(\phi)}{A_{\mathrm{LU}}^{\mathrm{A}}(\phi)}=\frac{A_{\mathrm{C} z z}^{\mathrm{A}(1)}}{A_{\mathrm{C}}^{\mathrm{A}(1)}} \sim \frac{2}{3} \frac{\left(-\tau+4 \xi \tau-3 \xi^{2}\right) G_{3}}{G_{1}-\frac{2}{3} \tau G_{3}} .
$$

For small values of $-\Delta^{2}$ and $\xi$ these ratios are rather small, e.g., $-\Delta^{2}=0.2[0.1] \mathrm{GeV}^{2}$ and $x_{\mathrm{B}}=0.1$ we find the number $\sim 0.2[0.08]$. A significant deviation from this prediction would indicate that $\mathcal{H}_{3}$ and/or $\mathcal{H}_{5}$ are comparable in size to $\mathcal{H}_{1}$. For instance, if $\mathcal{H}_{5}$ would be small and assuming that $\mathcal{H}_{3}=G_{3} / G_{1} \mathcal{H}_{1}$ this ratio is roughly double so large.

\footnotetext{
${ }^{12}$ The ratio of form factors is for small value of $\left|\Delta^{2}\right|$ given by the anomalous magnetic moment $F_{2} / F_{1} \approx 1.793(1-$ $\left.\Delta^{2} / 1.26\right)$, while the ratio of polarized to unpolarized quarks is estimated from the measured ratio of polarized to unpolarized structure functions in deep inelastic scattering which behave like $\sqrt{x_{\mathrm{B}}}$ for increasing $x_{\mathrm{B}}$, e.g., $g_{1} / F_{1} \sim 0.2$ for $x_{\mathrm{B}} \sim 0.1$.
} 


\begin{tabular}{|c||c|c||c|c||c||c|}
\hline \multicolumn{3}{|c||}{ all targets } & \multicolumn{2}{c||}{ proton } & neon & deuteron \\
\hline \hline model & $b_{\text {val }}$ & $b_{\text {sea }}$ & $B_{\text {sea }}\left[\mathrm{GeV}^{-2}\right]$ & $\kappa_{\text {sea }}$ & $B_{\text {sea }}\left[\mathrm{GeV}^{-2}\right]$ & $B_{\text {sea }}\left[\mathrm{GeV}^{-2}\right]$ \\
\hline \hline $\mathrm{A}$ & 1 & $\infty$ & 9 & 0 & 9 & 20 \\
\hline $\mathrm{B}$ & $\infty$ & $\infty$ & 9 & -3 & 12 & 30 \\
\hline $\mathrm{C}$ & 1 & 1 & 5 & 0 & 6 & 15 \\
\hline
\end{tabular}

Table 2: Parameter sets for $H$ and $H_{1}$ GPDs of a spin-0 nucleus, proton, and deuteron.

\subsection{Numerical results}

In the previous section we tried to convince the reader that a rather simple GPD ansatz explains the measured DVCS asymmetries. To avoid a misunderstanding, we do not claim that our estimates are precise. Rather we expect that more realistic GPD models yield large deviations from these naive estimates. Nevertheless we like to give an impression of this model dependence in the following. We also have to keep in mind that the kinematical approximations are rather rough. In the following we correct this and take the exact expressions for the $\mathrm{BH}$ cross section and $K$ factor, defined in Eq. (23) and the full $\xi$ and $\Delta^{2}$ dependences in the twist-two predictions of interference and squared DVCS term.

First we consider the beam spin asymmetry measured at HERMES. For the proton target we rely on the GPD models A, B, and C, employed in Ref. [7. The parameters of these three models are listed in Tab. 2. Compared to model A, the sea quarks are suppressed and enhanced in model $\mathrm{B}$ and $\mathrm{C}$, respectively, by a larger slope $B_{\text {sea }}$ and $b_{\text {sea }} \rightarrow \infty$. We also suppress in model $\mathrm{B}$ the valence quarks by taking $b_{\mathrm{val}} \rightarrow \infty$. At twist-three level the model $\mathrm{A}$ and $\mathrm{C}$ are based on the WW approximation, while in the model B quark-gluon-quark GPDs are added by 'hand', see Ref. [7] for details. We neglect the D-term, which affects the observable in question only sligthly at twist-three level. Taking the mean values of the 2000 data (138), the $\phi$-integrated beam spin asymmetry (119) at twist-two and twist-three level reads:

$$
A_{\mathrm{LU}}=\left\{\begin{array}{l}
{[-0.29,-0.25,-0.41]} \\
{[-0.29,-0.20,-0.37]}
\end{array}\right\} \text { for }\left\{\begin{array}{c}
\text { twist-two } \\
\text { twist-three }
\end{array}\right\} \text { and models }[\mathrm{A}, \mathrm{B}, \mathrm{C}] \text {. }
$$

As we realize the model B predictions are consistent with the experimental data, while the model A gives a slightly too high value for $\left|A_{\mathrm{LU}}\right|$. We remind that both model predictions are consistent with the 96/97 data. The large value of the model $\mathrm{C}$ prediction is caused by the sea quarks.

Now we present the predictions for the neon target at twist-two and -three level. Here again the sea quark contributions are relatively suppressed in model B and C, see Tab. 2, and we again add for the B model a quark-gluon-quark GPD contribution by 'hand', in the same way as it has been done for the proton. We find with these models:

$$
A_{\mathrm{LU}}^{\mathrm{Ne}}=\left\{\begin{array}{l}
{[-0.30,-0.28,-0.54]} \\
{[-0.34,-0.24,-0.49]}
\end{array}\right\} \text { for }\left\{\begin{array}{c}
\text { twist-two } \\
\text { twist-three }
\end{array}\right\} \text { and models }[\mathrm{A}, \mathrm{B}, \mathrm{C}]
$$

Again the model B prediction is on the one $\sigma$ level consistent with the experimental data (139).

Now we discuss estimates for an unpolarized and longitudinally polarized deuterium target in more detail. The models for the reduced deuteron GPD $H_{1}$ are listed in Tab. 2 and they share the same qualitative features as described above. The $D$-term contributions are neglected and we set for simplicity $\mu_{\text {sea }}=1$ and $\mathcal{Q}_{\text {sea }}=0$. To illustrate possible bound state effects, we sometimes take 

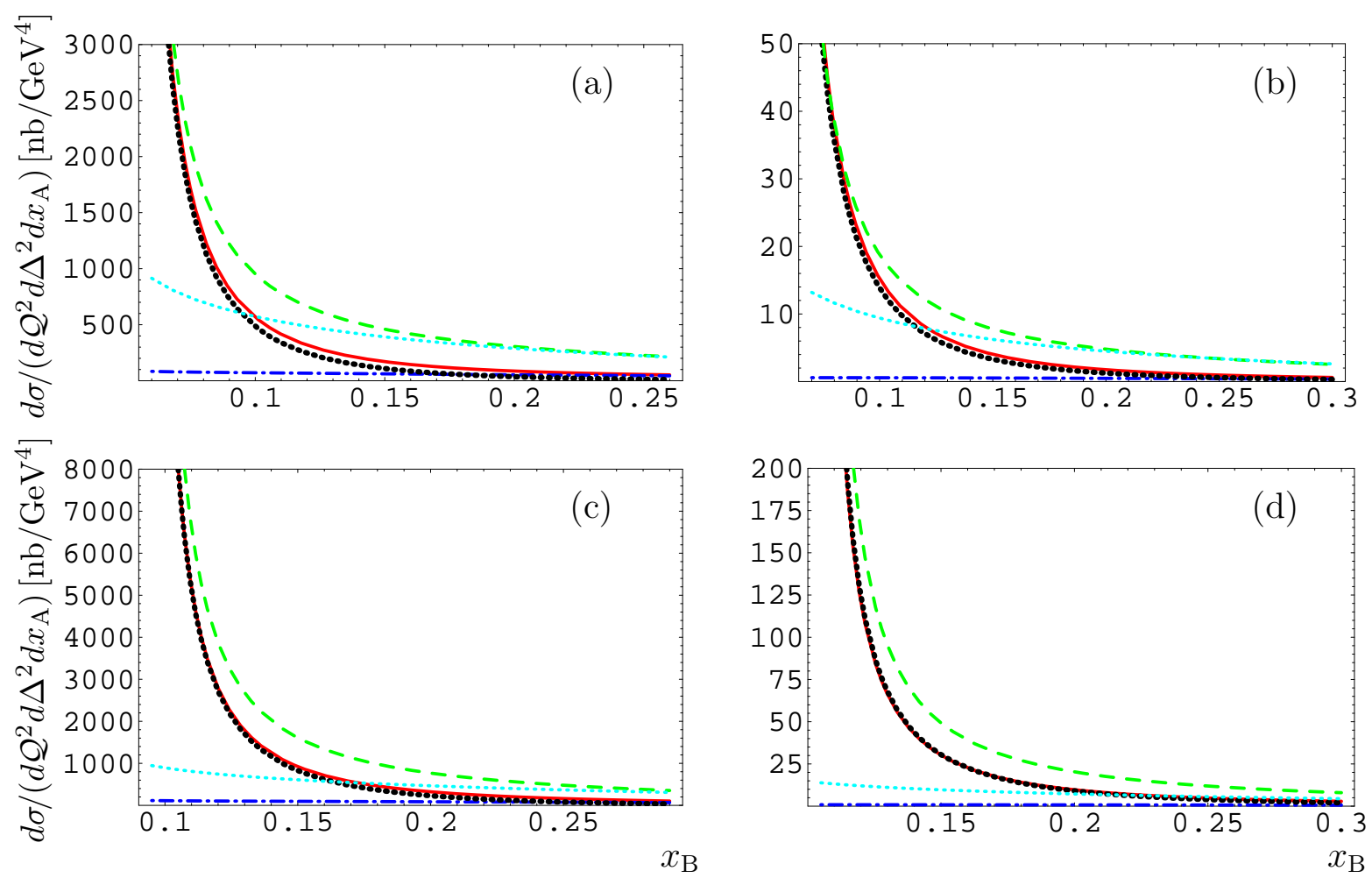

Figure 4: Model dependent estimates for the cross section, integrated over the azimuthal angle $\phi$, for the scattering of a positron [electron] beam on a deuteron target at $E_{e}=27.6[12] \mathrm{GeV}$, $\mathcal{Q}^{2}=2.5[2] \mathrm{GeV}^{2}, \Delta^{2}=-0.1 \mathrm{GeV}^{2}$ (a) [(c)] and $\Delta^{2}=-0.3 \mathrm{GeV}^{2}$ (b) [(d)]. The squared $\mathrm{BH}$ contribution is displayed as bold dotted line, the DVCS cross section as dash-dotted (dotted) and the leptoproduction cross section for positrons as solid (dashed) curves for model B (model C).

$H_{3}$ into account, equating the reduced GPD with that of $H_{1}$, or alternatively $H_{5}$, equating it with the antisymmetric part of $H_{1}\left(x, \eta, \Delta^{2}\right)$. These two additional sets will be denoted with a prime or a hat, respectively. As mentioned above, we neglect the kinematically suppressed contributions $H_{2}, H_{4}, \widetilde{H}_{2}$, and $\widetilde{H}_{3}$. The model of $\widetilde{H}_{1}$ is based on the GS A parameterization [61] with $\widetilde{b}_{\text {sea }}=1$. If not stated otherwise, we equate the slope parameters $\widetilde{B}_{\text {val }}=\widetilde{B}_{\text {sea }}=20 \mathrm{GeV}^{-2}$, which is roughly the size of the slope parameter for the charge form factor $G_{Q}$.

Let us first consider the size of the unpolarized cross sections for the positron and electron scattering off a deuteron target at HERMES and JLAB kinematics, respectively. It is demonstrated in Fig. 4 that at larger value of $x_{\mathrm{B}}$, i.e., smaller value of $y$, the cross section is dominated by the DVCS one, however, its size is comparably small. With decreasing $x_{\mathrm{B}}$, the $\mathrm{BH}$ cross section starts to dominate over the model dependent DVCS one. In model $\mathrm{C}$ the sea quarks also induce a growing DVCS cross section due to an associated large imaginary part in the DVCS amplitude. Typically, the ratio of BH to DVCS cross section and the total cross section is larger at JLAB compared to HERMES kinematics.

In Fig. 5 we show the model dependence for the predictions of the beam spin asymmetry $A_{\mathrm{L} \pm}$. In panel (a) we confront them with the preliminary HERMES measurement [27]. However, 

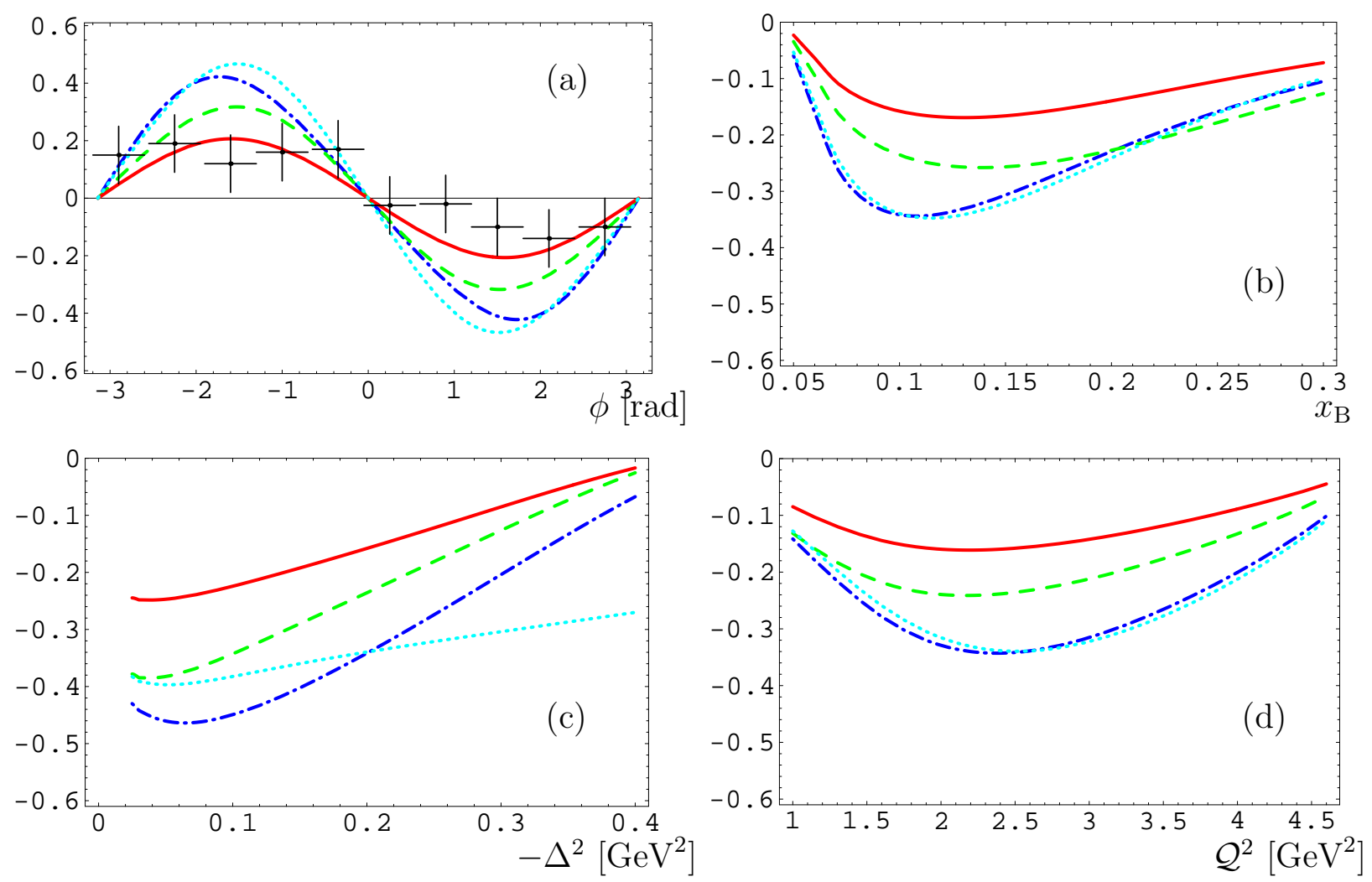

Figure 5: The beam spin asymmetry $\mathrm{A}_{\mathrm{L} \pm}(\phi)(\mathrm{a}), \mathrm{A}_{\mathrm{L} \pm}$ as function of $x_{\mathrm{B}}(\mathrm{b}),-\Delta^{2}(\mathrm{c})$, and $\mathcal{Q}^{2}(\mathrm{~d})$ [without evolution] for the scattering of a positron on a deuteron target at $E_{e}=27.6 \mathrm{GeV}$. The fixed kinematical variables are $x_{\mathrm{B}}=0.1, \mathcal{Q}^{2}=2.5 \mathrm{GeV}^{2}$, and $\Delta^{2}=-0.2 \mathrm{GeV}^{2}$, where the models A (dash-dotted), B (dashed), $\hat{B}$ (solid), and $\mathrm{B}^{\prime}$ (dotted) are specified in the text.

we remind that the analysis is based on two different data sets that, certainly, also contains incoherent scattering events. Model A (dash-dotted) gives a larger asymmetry than model B (dashed), caused by the larger sea quark content of the former model. Note that for the same reason, model $\mathrm{C}$ predicts an even larger asymmetry, which is not displayed. If we take into account the $H_{3}$ contribution via the $\mathrm{B}^{\prime}$ model (dotted), the beam spin asymmetry in panels $(\mathrm{a}, \mathrm{b}, \mathrm{d})$ is of the same size as the model A predictions. It is displayed in panel (c) that model $\mathrm{A}$ and $\mathrm{B}^{\prime}$ are distinguishable due to the $\Delta^{2}$ dependence. In absence of $H_{5}$ we observe for the typical HERMES kinematics only a rather small difference between the asymmetries $A_{\mathrm{LU}}$ and $A_{\mathrm{L} \pm}$. The latter asymmetry is sensitive to $H_{5}$ and we find a large reduction for the model $\hat{\mathrm{B}}$ (solid). We recall that the $\phi$-integrated asymmetries, displayed in panels (b)-(d), are smaller than the non-integrated asymmetries at $\phi=\pi / 2$. This integration also changes the $\Delta^{2}$ dependence ${ }^{13}$. For JLAB kinematics we find the same qualitative features, where of course for electrons the sign of the asymmetry is reversed. Let us add that within the model B we have a slightly larger value than in our result

\footnotetext{
${ }^{13}$ The unintegrated asymmetries (112) and (115) are roughly spoken proportional to $\sqrt{-\Delta^{2} / \mathcal{Q}^{2}}$ and thus will constantly decrease with decreasing $\left|\Delta^{2}\right|$. For the integrated asymmetry we rather observe a growing that comes from the $\phi$ integration, indicated in Eq. [119). Of course, in any case the asymmetry vanishes in the limit $\Delta^{2} \rightarrow \Delta_{\min }^{2}$
} 

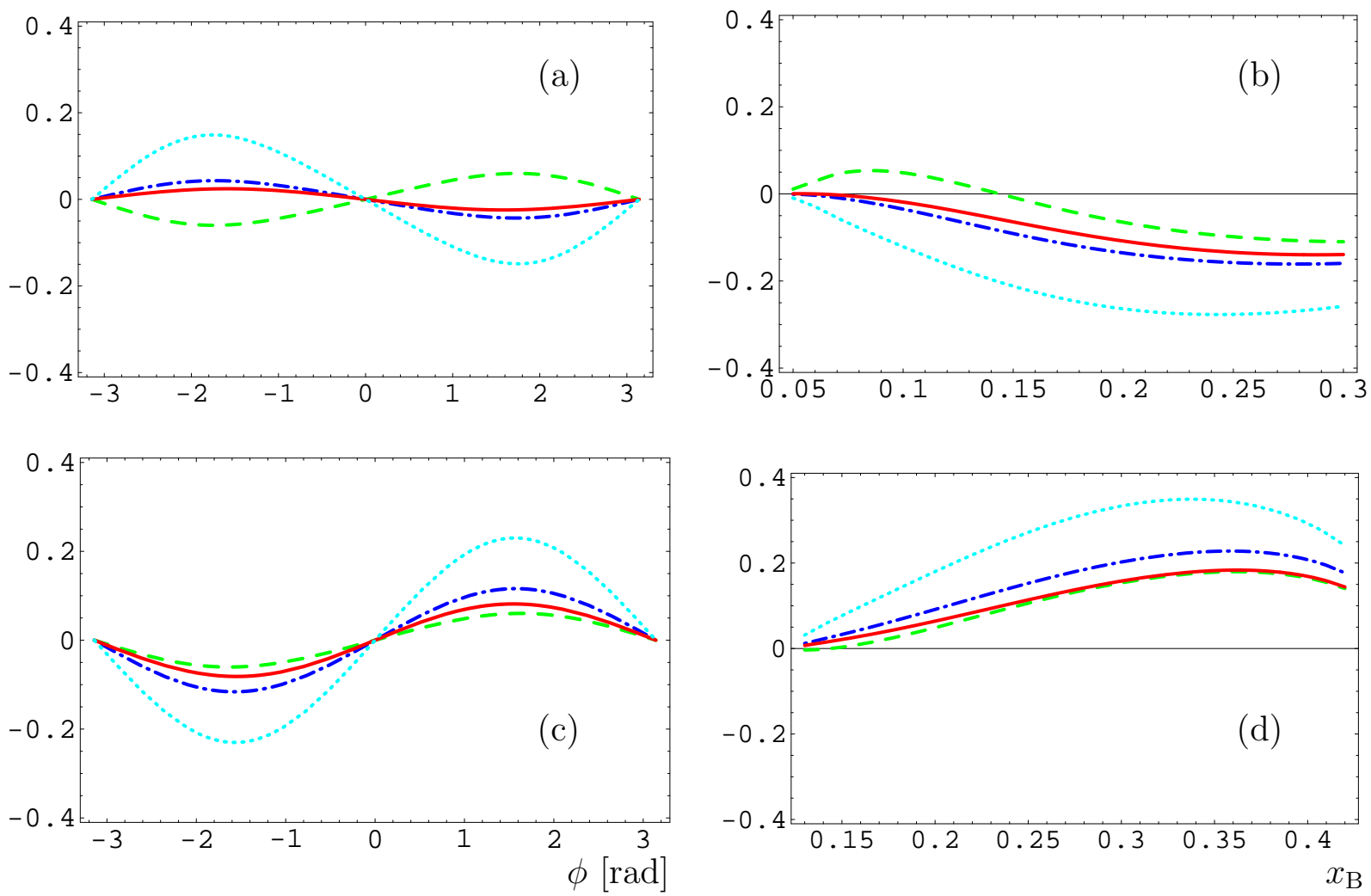

Figure 6: The longitudinal target asymmetry $A_{\mathrm{UL}}(\phi)(\mathrm{a}, \mathrm{c})$ and $A_{\mathrm{UL}}(\mathrm{b}, \mathrm{d})$ versus $\phi$ and $x_{\mathrm{B}}$, respectively. For HERMES $(\mathrm{a}, \mathrm{b})[\mathrm{JLAB} @ 12 \mathrm{GeV}(\mathrm{c}, \mathrm{d})]$ kinematics we take $x_{\mathrm{B}}=0.1\left[x_{\mathrm{B}}=0.2\right]$, $-\Delta^{2}=0.2 \mathrm{GeV}^{2}$ and $\mathcal{Q}^{2}=2.5 \mathrm{GeV}^{2}$ as well as employ the model A with $\widetilde{B}_{\text {val }}=\widetilde{B}_{\text {sea }}=20 \mathrm{GeV}^{-2}$ (dash-dotted), $\widetilde{B}_{\text {val }}=30 \mathrm{GeV}^{-2}, \widetilde{B}_{\text {sea }}=10 \mathrm{GeV}^{-2}$ (dashed), $\widetilde{B}_{\text {val }}=10 \mathrm{GeV}^{-2}, \widetilde{B}_{\text {sea }}=30 \mathrm{GeV}^{-2}$ (dotted), and $\hat{\mathrm{B}}$ with $\widetilde{B}_{\text {val }}=\widetilde{B}_{\text {sea }}=20 \mathrm{GeV}^{-2}$ (solid).

(139) of the previous Section, caused by both kinematical and sea quark contributions. The other models induce the typical effects, we have already discussed:

$$
\left\{\begin{array}{l}
A_{\mathrm{LU}} \\
A_{\mathrm{L} \pm}
\end{array}\right\}=\left\{\begin{array}{l}
{[-0.37,-0.26,-0.23,-0.34]} \\
{[-0.34,-0.24,-0.16,-0.34]}
\end{array}\right\} \text { for model }\left[\mathrm{A}, \mathrm{B}, \hat{\mathrm{B}}, \mathrm{B}^{\prime}\right]
$$

These results will also be altered by twist-three contributions, i.e., change of the normalization and excitation of higher harmonics. A quantitative estimate of such contributions is beyond the scope of this paper. One might expect a similar change as in the case of a proton target, discussed in great detail in Ref. [7.

As discussed above, see also Eq. (153), the size of the longitudinal target spin asymmetry $A_{\mathrm{UL}}$ is rather model dependent and allows to access the imaginary part of the parity odd $\mathrm{CFF} \widetilde{\mathcal{H}}_{1}$. This is demonstrated for HERMES and JLAB kinematics in the panels (a,b) and (c,d), respectively, of Fig. 6. Here we set $x_{\mathrm{B}}=0.1$ and $x_{\mathrm{B}}=0.2$, respectively, as well as $-\Delta^{2}=0.2 \mathrm{GeV}^{2}$ and $\mathcal{Q}^{2}=2.5 \mathrm{GeV}^{2}$. For models A (dash-dotted) and B (solid) with same $\widetilde{\mathcal{H}}_{1}$, taking $\widetilde{B}_{\text {val }}=\widetilde{B}_{\text {sea }}=20 \mathrm{GeV}^{-2}$, we find a small asymmetry. Within this model parameter the contribution of $\widetilde{\mathcal{H}}_{1}$ is negligible, since its valence and sea quark contributions partly cancel each other. Thus, we observe in agreement 

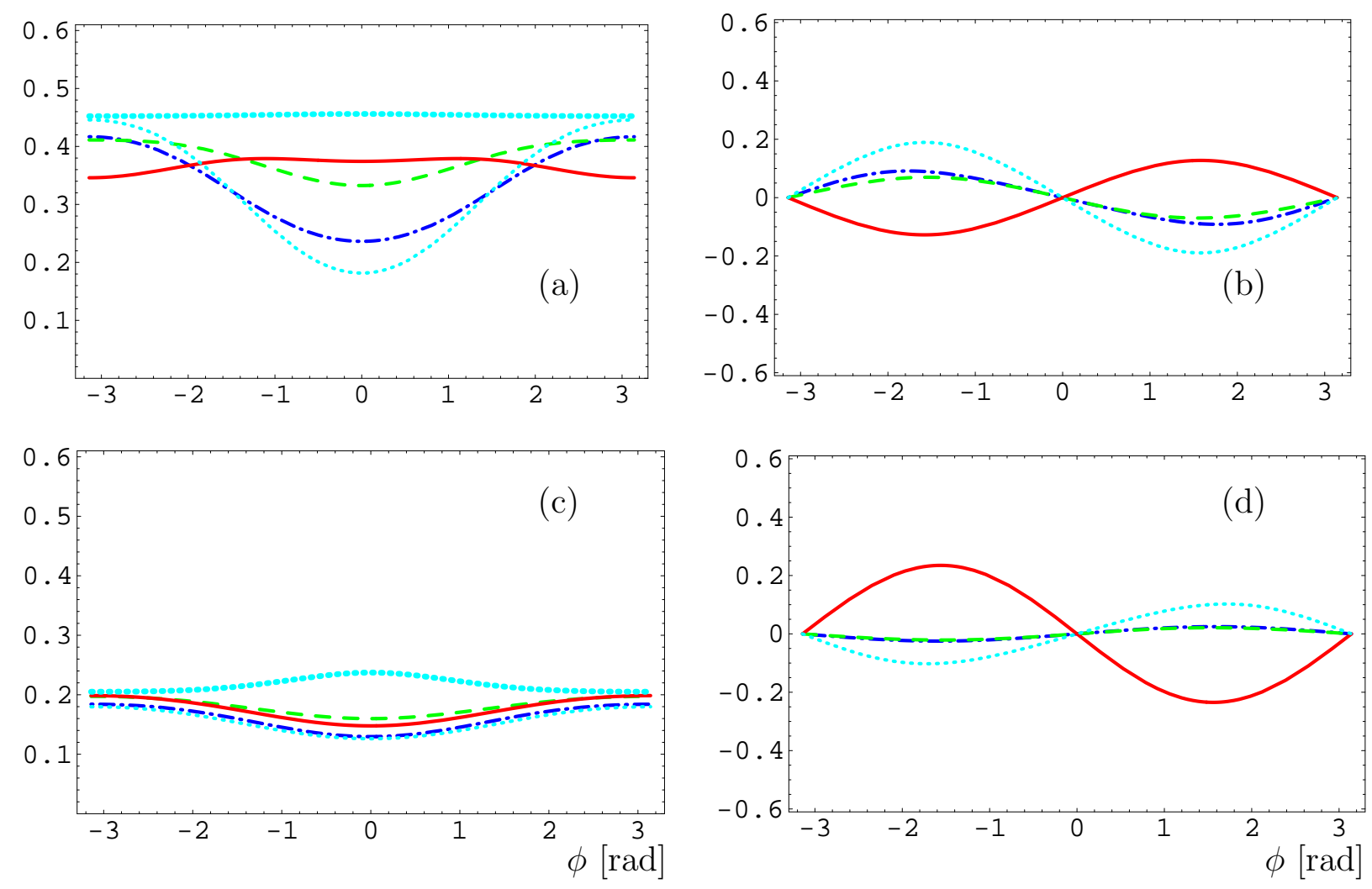

Figure 7: Tensor asymmetries $A_{z z}(\phi)(\mathrm{a}, \mathrm{c})$ and $A_{\mathrm{L} z z}(\phi)(\mathrm{b}, \mathrm{d})$ for HERMES (a,b) and JLAB@12 $\mathrm{GeV}(\mathrm{c}, \mathrm{d})$ kinematics within the same GPD models as in Fig. 5 and mean values as in Fig. 6. The thick dotted line in panels $(\mathrm{a}, \mathrm{c})$ shows only the $\mathrm{BH}$ cross section.

with Eq. (153) that the asymmetry has the same sign as the beam spin asymmetry and generally increases with growing $x_{\mathrm{B}}$, as it is displayed in panels (b) and (c). This is mainly caused by the fact that the asymmetry is proportional to $1 / y$. Thus, comparing panel (a) and (c), we realize that for the typical JLAB kinematics (larger $x_{\mathrm{B}}$ ) our estimates for $\left|A_{\mathrm{UL}}(\phi=\pi / 2)\right|$ are slightly larger than for the HERMES one. If the polarized valence quarks are relatively suppressed with respect to the sea quarks, displayed by the dashed line, the negative sea quark contribution takes over and induces the sign change in panel (a). For larger value of $x_{\mathrm{B}}$, see panel (b), the asymmetry turns out to be positive. For the contrary case that the valence quark contribution is enhanced (dotted line), the target spin asymmetry becomes sizably large.

The sign of the charge asymmetry $A_{\mathrm{C}}$ is not predictable. For the mean values used above, we find that the model $\mathrm{A}$ gives an asymmetry that is negligibly small, the $\mathrm{B}$ models provide a positive and the $\mathrm{C}$ model a large negative contribution, where no $D$-term has been taken into account. This observation is in qualitative agreement with our results for the proton target and affirms once more that an access to the $D$-term contribution is intricate.

The tensor asymmetry $A_{z z}(\phi)$, cf. Eq. (116), of an unpolarized positron and electron beam is displayed in Fig. [7(a) and (c) for the same HERMES and JLAB@12 GeV kinematics as in Fig. 6. respectively. The thick dotted line shows the pure BH contribution, which is in correspondence with Eq. (128) almost flat for HERMES kinematics (a). In the case of JLAB kinematics (b) its 
contribution is much smaller compared to the approximation (128), which is caused by higher order terms in $\xi$ and $\tau$ that have not been taken into account [see analogous discussion of Eq. (153)]. Subtraction of this contribution gives a dominant $\cos (\phi)$ contribution that is proportional to the real part of the linear combination (129). As in the case of the charge asymmetry the sign depends on the details of GPDs. In panels $(\mathrm{b}, \mathrm{d})$ we have ploted the tensor asymmetry $A_{\mathrm{L} z z}(\phi)$, cf. Eq. (131), for a polarized lepton beam, which is essentially given by a $\sin (\phi)$ harmonic. Its amplitude is proportional to the imaginary part of CFFs combination that enters Eq. (132). If both CFFs $\mathcal{H}_{3}$ and $\mathcal{H}_{5}$ are small, as it is realized in models A (dash-dotted) and B (dashed), we find only a small asymmetry, which has the same sign as the beam spin asymmetries. This is in line with approximation (154). This tensor asymmetry is rather sensitive to bound state effects. For instance, in the case that there is an important $\Im m \mathcal{H}_{3}$ contribution the asymmetry becomes sizable. On the other hand, if we assume a large positive $\Im m \mathcal{H}_{5}$ and a negligible $\Im m \mathcal{H}_{3}$ contribution, i.e., taking model B' (solid), we find a sizable asymmetry that has a reversed sign.

\section{Summary}

In this paper we considered the leptoproduction of a photon on nuclei up to spin- 1 . For spin-0 and spin- $1 / 2$ targets the theoretical predictions in terms of nuclei GPDs simply follow from the known results, presented in Refs. 6, 53, 7] at the twist-three level, by appropriate replacements of form factors, GPDs, and kinematical variables. As a new result we added to this collection the azimuthal angular dependence of the leptoproduction cross section of a photon on a spin-1 target at twist-two level to leading order in perturbation theory. Since the hard-scattering part is for all targets unique, the result for the considered harmonics can immediately be extended to NLO. At this order also a gluon tensor contribution appears, which induces a $\cos (3 \phi)$ and $\cos (2 \phi)$ harmonic in the interference and squared DVCS term, respectively. We should add that the elaboration of the twist-three sector, providing the zero and second harmonics in the interference term and the first ones in the DVCS cross section, is straightforward in our covariant formalism.

For a spin-1 target nine CFFs enter the twist-two sector, which are given as a convolution with nine GPDs. From the theoretical point of view, the measurement of the imaginary and real part of all these CFFs is possible for the case of a polarized lepton beam and target, with an adjustable quantization direction, for both kinds of leptons is available. Moreover, an appropriate Fourier analysis allows to eliminate the twist-three contamination. In this way one has the maximal access to the twist-two deuteron GPDs, given at the diagonal $x=\xi$ and as convolution.

An important issue is the contamination of the leading twist-two prediction by power suppressed contributions. Naively, one would expect that such contributions scale with $\Delta^{2} / \mathcal{Q}^{2}$ and $M_{A}^{2} / \mathcal{Q}^{2}$. Fortunately, within our model we showed that the so-called target mass corrections have the same $A$ scaling as the leading twist-two contributions. A more detailed investigation of power suppressed contributions would be very desired.

Certainly, a dedicated experiment is required to measure all CFFs. Having on hand only an unpolarized target one can access the beam spin and charge asymmetry via the Fourier coefficients $s_{1, \text { unp }}^{\mathcal{I}}$ and $c_{1 \text {,unp }}^{\mathcal{I}}$, given as a certain linear combinations of $\mathcal{H}_{1}, \mathcal{H}_{3}$, and $\mathcal{H}_{5}$ CFFs. A longitudinally polarized target allows to measure four further independent observables. Two of them, e.g., the tensor polarization combined with beam spin or charge asymmetry, are predicted by another linear combination of the afore mentioned three CFFs, entering the Fourier coefficients $s_{1, \mathrm{LLP}}^{\mathcal{I}}$ and $c_{1, \mathrm{LLP}}^{\mathcal{I}}$. 
Unfortunately, such experiments do not allow to access all three GPDs in question. The remaining two observables are related to the target spin flip odd asymmetries, given by the FCs $s_{1, \mathrm{LP}}^{\mathcal{I}}$ and $c_{1, \mathrm{LP}}^{\mathcal{I}}$. In a single target spin flip experiment one can measure $s_{1, \mathrm{LP}}^{\mathcal{I}}$ and get access to the imaginary

part of $\widetilde{\mathcal{H}}_{1}$. On the other hand, combining the data for polarized target with charge asymmetry or the flip of the beam helicity allow then to measure $c_{1, \mathrm{IP}}^{\mathcal{I}}$ and so also the real part of $\widetilde{\mathcal{H}}_{1}$. In all these observables, we discussed so far, the remaining five CFFs are kinematically suppressed or do not enter the theoretical predictions.

To estimate the size of different observables, we proposed a simple model for nuclei GPDs that is based on the $A$-scaling of the longitudinal degrees of freedom and a $\Delta^{2}$ factorized ansatz of the nucleon GPDs. For a spin-0 or $-1 / 2$ nucleus target, this ansatz is sufficient to fix all reduced GPDs in terms of the proton ones (of course, they are also unknown). The remaining degree of freedom concerns mainly the form factors, especially, in the sea quark sector. Of course, binding effects should alter this oversimplified ansatz, e.g., we expect that t-channel exchange forces can induce an important contribution in the 'exclusive' region of GPDs. Such effects might be visible by a precise measurement of single spin and charge asymmetries. For a spin-1 target the large number of GPDs opens a new window to study nuclear binding effects. Compared to deep inelastic scattering or elastic lepton-deuteron scattering, they provide additional information, contained in the CFFs. Beside the modifaction of the the scaling law for the CFFs $\mathcal{H}_{1}$ and $\widetilde{\mathcal{H}}_{1}$, they induce non-vanishing CFFs $\mathcal{H}_{3}$ and $\mathcal{H}_{5}$. We conclude that nucleus GPDs give a new testing ground for non-perturbative methods that have been used in nuclear physics for the calculation of form factors, see reviews 30, 31.

Finally, we estimated for the typical kinematics of present fixed target experiments, different asymmetries and showed that a qualitative understanding of them is possible by means of analytical formulae, which are obtained by a simplification of kinematics and GPD models. For a deuteron target such an approximation allows to discuss the contribution of the CFFs $\mathcal{H}_{3}$ and $\mathcal{H}_{5}$. The LO analyses of the pioneering measurements of DVCS [19, 21, 22] on the proton suggest for $-\Delta^{2} \sim 0.3 \mathrm{GeV}^{2}$ the dominance of valence-quark GPDs in the valence quark region with no essential enhancement by the skewedness effect. Assuming the same features also for the nuclei GPDs, our estimates agree with the $\phi$-integrated beam spin asymmetries measured on neon and deuteron targets. In the latter case this asymmetry is already sensitive to the CFFs $\mathcal{H}_{3}$ and $\mathcal{H}_{5}$. A measurement of the tensor polarization would certainly provide a new constraint for these CFFs.

\section{Acknowledgements}

We are very grateful to M. Diehl, A. Freund, and A. Schäfer for useful discussions on different theoretical issues. We are indebted to M. Amarian, B. Krauss, and R. Shanidze for discussions of the experimental settings and to J. Ball for providing us the parameters of deuteron form factor parameterization. One of us (A.K.) would like to thank the Studienstiftung des deutschen Volkes for financial support. He also wants to thank the CTP (MIT), especially, Prof. Jaffe and Prof. Negele for kind hospitality and many useful discussions.

Note added: Recently there appeared a note 62 where DVCS on a spin-0 nucleus has been considered in the impuls approximation. This result overlaps with a part of our analysis in 
Sections 4 and 5 .

\section{A Parameterization of the electromagnetic deuteron form factors}

The set of form factors $G_{1}, G_{2}$, and $G_{3}$ may be expressed by the charge monopole, magnetic dipole, and charge quadrupole form factors:

$$
\begin{aligned}
G_{C}\left(\Delta^{2}\right) & =\left(1-\frac{2 \tau}{3}\right) G_{1}\left(\Delta^{2}\right)+\frac{2 \tau}{3}\left[G_{2}\left(\Delta^{2}\right)-(1-\tau) G_{3}\left(\Delta^{2}\right)\right] \\
G_{M}\left(\Delta^{2}\right) & =G_{2}\left(\Delta^{2}\right) \\
G_{Q}\left(\Delta^{2}\right) & =G_{1}\left(\Delta^{2}\right)-G_{2}\left(\Delta^{2}\right)+(1-\tau) G_{3}\left(\Delta^{2}\right)
\end{aligned}
$$

with $\tau=\frac{\Delta^{2}}{4 M_{\mathrm{A}}^{2}}$. The normalizations of these form factors read

$$
G_{C}(0)=1, \quad G_{M}(0)=\mu_{d}=1.714, \quad G_{Q}(0)=Q_{d}=25.83 .
$$

Their parameterization, which is inspired by the counting rules for large $-\Delta^{2}$ was taken from Ref. [54]:

$$
\begin{aligned}
& G_{C}\left(\Delta^{2}\right)=\frac{G_{D}^{2}\left(\frac{\Delta^{2}}{4}\right)}{1-2 \tau}\left[\left(1+\frac{2}{3} \tau\right) g_{00}^{+}+\frac{8}{3} \sqrt{-2 \tau} g_{+0}^{+}-\frac{2}{3}(1+2 \tau) g_{+-}^{+}\right] \\
& G_{M}\left(\Delta^{2}\right)=\frac{G_{D}^{2}\left(\frac{\Delta^{2}}{4}\right)}{1-2 \tau}\left[2 g_{00}^{+}-\frac{2(1+2 \tau)}{\sqrt{-2 \tau}} g_{+0}^{+}-2 g_{+-}^{+}\right] \\
& G_{Q}\left(\Delta^{2}\right)=\frac{G_{D}^{2}\left(\frac{\Delta^{2}}{4}\right)}{1-2 \tau}\left[-g_{00}^{+}+\sqrt{-\frac{2}{\tau}} g_{+0}^{+}+\frac{1-\tau}{\tau} g_{+-}^{+}\right] .
\end{aligned}
$$

Here we use the standard dipole parameterization for the nucleon form factor

$$
G_{D}\left(\Delta^{2}\right)=\left(1-\frac{\Delta^{2}}{0.71 \mathrm{GeV}^{2}}\right)^{-2}
$$

and the helicity transition amplitudes in the infinite momentum frame [63] are given by

$$
g_{00}^{+}=\sum_{i=1}^{4} \frac{a_{i}}{\alpha_{i}^{2}-\Delta^{2}}, \quad g_{+0}^{+}=\sqrt{-\Delta^{2}} \sum_{i=1}^{4} \frac{b_{i}}{\beta_{i}^{2}-\Delta^{2}}, \quad g_{+-}^{+}=-\Delta^{2} \sum_{i=1}^{4} \frac{c_{i}}{\gamma_{i}^{2}-\Delta^{2}} .
$$

Counting rules predict for large $-\Delta^{2}$ the following behavior

$$
g_{00}^{+} \sim(-\Delta)^{-2}, \quad g_{+0}^{+} \sim(-\Delta)^{-3}, \quad g_{+-}^{+} \sim(-\Delta)^{-4},
$$

which gives together with the static properties (159) six constraints for twentyfour fitting parameters:

$$
\sum_{i=1}^{4} \frac{a_{i}}{\alpha_{i}^{2}}=1, \sum_{i=1}^{4} \frac{b_{i}}{\beta_{i}^{2}}=\frac{2-\mu_{d}}{2 \sqrt{2} M_{d}}, \sum_{i=1}^{4} \frac{c_{i}}{\gamma_{i}^{2}}=\frac{1-\mu_{d}-Q_{d}}{4 M_{d}^{2}}, \sum_{i=1}^{4} b_{i}=\sum_{i=1}^{4} c_{i}=\sum_{i=1}^{4} c_{i} \gamma_{i}^{2}=0 .
$$




\begin{tabular}{|l|c|c|c|c|}
\hline \multicolumn{1}{|r|}{$i=$} & 1 & \multicolumn{1}{|c|}{2} & 3 & 4 \\
\hline \hline$a_{i}\left[\mathrm{fm}^{-2}\right]$ & 1.57057 & 12.23792 & -42.04576 & Eq. (163) \\
\hline$b_{i}\left[\mathrm{fm}^{-1}\right]$ & 0.07043 & 0.14443 & Eq. (163) & Eq. (163) \\
\hline$c_{i}$ & -0.16577 & Eq. (163) & Eq. (163) & Eq. (163) \\
\hline$\alpha_{i}^{2}\left[\mathrm{fm}^{-2}\right]$ & 1.52501 & Eq. (164) & Eq. (164) & 23.20415 \\
\hline$\beta_{i}^{2}\left[\mathrm{fm}^{-2}\right]$ & 43.67795 & Eq. (164) & Eq. (164) & 2.80716 \\
\hline$\gamma_{i}^{2}\left[\mathrm{fm}^{-2}\right]$ & 1.87055 & Eq. (164) & Eq. (164) & 41.1294 \\
\hline
\end{tabular}

Table 3: Fitting parameter sets for the electromagnetic Deuteron form factors.

To reduce this set to twelve parameters, one may introduce for each group $\alpha_{i}, \beta_{i}$, and $\gamma_{i}$ the algebraic relations:

$$
\alpha_{i}^{2}=\alpha_{1}^{2}+\left(\alpha_{4}^{2}-\alpha_{1}^{2}\right) \frac{i-1}{3} \quad \text { for } \quad i=1, \ldots, 4 .
$$

The fitting parameters are taken from Ref. [55] and are given in Tab. 3 .

\section{B Results for the Fourier coefficients}

Below the twist-two results for unpolarized and longitudinally polarized target are listed. All results have been expanded for small $\tau=\frac{\Delta^{2}}{4 M^{2}}$, i.e., for $\Delta^{2} \ll M^{2}$. Terms proportional to $\mathcal{H}_{3}, G_{3}$ are given up to order $\mathcal{O}(\tau)$, because $G_{3}$ is at $\Delta^{2}=0$ roughly 20 times larger than the other form factors. The BH amplitude squared has been exactly calculated. However, the results are very cumbersome and, thus, they are only presented in leading order of $1 / \mathcal{Q}^{2}$. We note that the rather lengthy results for $\mathcal{M}_{0, \mathrm{LLP}}^{\mathcal{I}, \mathrm{DVCS}}$ and $C_{0, \mathrm{LLP}}^{\mathrm{BH}}$ can be obtained through the much shorter results for unpolarized and transverse-transverse coefficients via equations (44).

\section{B.1 Interference term}

Here we give the matrices $\mathcal{M}_{1 \text {,unp }}^{\mathcal{I}}, \mathcal{M}_{1, \mathrm{LP}}^{\mathcal{I}}$, and $\mathcal{M}_{1, \mathrm{TTP}}^{\mathcal{I}}$, which appear in Eq. (46).

$$
\mathcal{M}_{1, \text { unp }}^{\mathcal{I}}=\frac{1}{9}\left(\begin{array}{ccc}
9 & 0 & -6 \tau \\
0 & 0 & 0 \\
-6 \tau & 0 & 12 \tau^{2} \\
-6 \xi & 0 & 12 \xi \tau \\
9 \xi^{2} & -6 \xi^{2} & 2\left(\xi^{2}(3-6 \tau)+\tau\right) \\
0 & 6 \xi & 0 \\
0 & 0 & 0 \\
0 & 0 & 0 \\
0 & 0 & 0
\end{array}\right)
$$




$$
\begin{aligned}
& \mathcal{M}_{1, \mathrm{LP}}^{\mathcal{I}}=\frac{1}{6(1+\xi)}\left(\begin{array}{ccc}
0 & 6 \xi & 0 \\
0 & 3 \xi^{2} & 0 \\
0 & -6 \xi \tau & 0 \\
0 & -3 \xi^{2} & 0 \\
0 & -2 \xi & 0 \\
6\left(1+\xi-\xi^{2}\right) & 6 \xi^{2} & -6 \xi^{2}(1-2 \tau)-6 \tau \\
-24 \xi^{2} & 0 & 24 \xi^{2} \tau \\
0 & 024 \xi^{2} \tau & \\
-6(1-\xi) \xi^{2} & -6 \xi^{3} & 6 \xi\left(\xi^{2}(1-2 \tau)+\tau+\xi \tau\right)
\end{array}\right) \\
& \mathcal{M}_{1, \mathrm{TTP} \Sigma}^{\mathcal{I}}=\frac{1}{6(1+\xi)^{2}}\left(\begin{array}{ccc}
6(1+(2-\xi) \xi) & 6 \xi^{2} & -6 \xi^{2}(1-3 \tau)-6 \tau \\
6(1-\xi) \xi^{2} & 6 \xi^{3} & 6 \xi\left(-\left(\xi^{2}(1-2 \tau)-\tau-\xi \tau\right)\right. \\
-6 \xi^{2}(1-3 \tau)-6 \tau & -6 \xi(1+2 \xi) \tau & 12 \tau\left(\xi^{2}(1-2 \tau)+\tau\right) \\
-6(1-\xi) \xi^{2} & -6 \xi^{3} & 6 \xi\left(\xi^{2}(1-2 \tau)+\tau+\xi \tau\right) \\
-2(1+(2-\xi) \xi) & -2 \xi^{2} & 2\left(\xi^{2}(1-3 \tau)+\tau\right) \\
0 & 3 \xi(1+\xi)(2+\xi) & 0 \\
0 & -12 \xi^{3} & 0 \\
0 & 12 \xi^{3} & 0
\end{array}\right)
\end{aligned}
$$

\section{B.2 DVCS amplitude squared}

The matrices $\mathcal{M}_{0, i}^{\mathrm{DVCS}}$, entering Eq. (48), for $i \in\{\mathrm{unp}, \mathrm{TP}-, \mathrm{LLP}, \mathrm{LTP}+, \mathrm{TTP} \Sigma, \mathrm{TTP} \Delta\}$ are of the form

$$
\mathcal{M}_{0, i}^{\mathrm{DVCS}}=\left\{\begin{array}{cc}
A_{0, i}^{\mathrm{DVC}} & 0 \\
0 & B_{0, i}^{\mathrm{DVCS}}
\end{array}\right\},
$$

with $A_{0, i}^{\text {DVCS }}$ being a $5 \times 5$ and $B_{0, i}^{\text {DVCS }}$ being a $4 \times 4$ matrix. The matrices $\mathcal{M}_{0, j}^{\text {DVCS }}$ for $j \in$ $\{\mathrm{LP}, \mathrm{TP}+, \mathrm{TTP} \pm\}$ have the building blocks

$$
\mathcal{M}_{0, j}^{\mathrm{DVCS}}=\left\{\begin{array}{cc}
0 & C_{0, j}^{\mathrm{DVCS}} \\
D_{0, j}^{\mathrm{DVCS}} & 0
\end{array}\right\},
$$

where $C_{0, j}^{\mathrm{DVCS}}$ is a $5 \times 4$ and $D_{0, j}^{\mathrm{DVCS}}$ is a $4 \times 5$ matrix.

We list only the submatrices $A_{0, i}^{\mathrm{DVCS}}, B_{0, i}^{\mathrm{DVCS}}, C_{0, j}^{\mathrm{DVCS}}, D_{0, j}^{\mathrm{DVCS}}$ that are needed for an unpolarized and longitudinally polarized target:

$$
\begin{aligned}
A_{0, \text { unp }}^{\text {DVCS }} & =\frac{1}{9}\left(\begin{array}{ccccc}
18 & 0 & -12 \tau & -12 \xi & 18 \xi^{2} \\
0 & -12 \xi^{2} & 0 & 0 & -24 \xi^{2} \\
-12 \tau & 0 & 24 \tau^{2} & 24 \xi \tau & 4\left(\xi^{2}(3-6 \tau)+\tau\right) \\
-12 \xi & 0 & 24 \xi \tau & 12 \xi^{2} & -4 \xi\left(2+3 \xi^{2}\right) \\
18 \xi^{2} & -24 \xi^{2} & 4\left(\xi^{2}(3-6 \tau)+\tau\right) & -4 \xi\left(2+3 \xi^{2}\right) & 4-24 \xi^{2}+6 \xi^{4}
\end{array}\right) \\
B_{0, \text { unp }}^{\text {DVCS }} & =\frac{1}{9}\left(\begin{array}{cccc}
12\left(1-\xi^{2}\right) & -48 \xi^{2} & 0 & -12 \xi^{2} \\
-48 \xi^{2} & 0 & 0 & 0 \\
0 & 0 & 0 & 48 \xi^{3} \\
-12 \xi^{2} & 0 & 48 \xi^{3} & -12 \xi^{2}\left(1+\xi^{2}\right)
\end{array}\right)
\end{aligned}
$$




$$
\begin{aligned}
& C_{0, \mathrm{LP}}^{\mathrm{DVCS}}=\frac{1}{3(1+\xi)}\left(\begin{array}{cccc}
6\left(1+\xi-\xi^{2}\right) & -24 \xi^{2} & 0 & -6(1-\xi) \xi^{2} \\
3(1-\xi) \xi^{2} & -12 \xi^{3} & -12 \xi^{3} & 3(1-\xi)^{2} \xi^{2} \\
-6 \xi^{2}(1-2 \tau)-6 \tau & 24 \xi^{2} \tau & 24 \xi^{2} \tau & 6 \xi(\tau+\xi(\xi+\tau-2 \xi \tau)) \\
-3(1-\xi) \xi^{2} & 12 \xi^{3} & 12 \xi^{3} & -3(1-\xi)^{2} \xi^{2} \\
-2(1+(1-\xi) \xi) & 8 \xi^{2} & 0 & 2(1-\xi) \xi^{2}
\end{array}\right) \\
& D_{0, \mathrm{LP}}^{\mathrm{DVCS}}=\frac{1}{3(1+\xi)}\left(\begin{array}{ccccc}
6\left(1+\xi-\xi^{2}\right) & 3(1-\xi) \xi^{2} & -6 \xi^{2}(1-2 \tau)-6 \tau & -3(1-\xi) \xi^{2} & -2(1+(1-\xi) \xi) \\
-24 \xi^{2} & -12 \xi^{3} & 24 \xi^{2} \tau & 12 \xi^{3} & 8 \xi^{2} \\
0 & -12 \xi^{3} & 24 \xi^{2} \tau & 12 \xi^{3} & 0 \\
-6(1-\xi) \xi^{2} & 3(1-\xi)^{2} \xi^{2} & 6 \xi(\tau+\xi(\xi+\tau-2 \xi \tau)) & -3(1-\xi)^{2} \xi^{2} & 2(1-\xi) \xi^{2}
\end{array}\right)
\end{aligned}
$$




$$
\begin{aligned}
& A_{0, \mathrm{TTP} \Sigma}^{\mathrm{DVCS}}=\frac{1}{9(1+\xi)^{2}}\left(\begin{array}{cc}
9(2+2(2-\xi) \xi) & 18(1-\xi) \xi^{2} \\
18(1-\xi) \xi^{2} & -9(1-\xi)^{2} \xi^{2} \\
18\left(-\left(\xi^{2}(1-3 \tau)\right)-\tau\right) & 18 \xi\left(-\left(\xi^{2}(1-2 \tau)\right)-\tau-\xi \tau\right) \\
-18(1-\xi) \xi^{2} & 9(1-\xi)^{2} \xi^{2} \\
-6(1+(2-\xi) \xi) & -6(1-\xi) \xi^{2}
\end{array}\right. \\
& \begin{array}{c}
18\left(-\left(\xi^{2}(1-3 \tau)\right)-\tau\right) \\
18 \xi\left(-\left(\xi^{2}(1-2 \tau)\right)-\tau-\xi \tau\right)
\end{array} \\
& 36 \tau\left(\xi^{2}(1-2 \tau)+\tau\right) \\
& 18 \xi(\tau+\xi(\xi+\tau-2 \xi \tau)) \\
& 6\left(\xi^{2}(1-3 \tau)+\tau\right) \\
& -18(1-\xi) \xi^{2} \\
& 18 \xi(\tau+\xi(\xi+\tau-2 \xi \tau)) \quad 6\left(\xi^{2}(1-3 \tau)+\tau\right) \\
& -9(1-\xi)^{2} \xi^{2}
\end{aligned}
$$

$$
B_{0, \mathrm{TTP} \Sigma}^{\mathrm{DVCS}}=\frac{1}{9(1+\xi)^{2}}\left(\begin{array}{cccc}
9(1+\xi)^{2}\left(2-\xi^{2}\right) & -36 \xi^{2}(1+\xi)(2+\xi) & -36 \xi^{3}(1+\xi) & -9(1-\xi) \xi^{2}(1+\xi)^{2} \\
-36 \xi^{2}(1+\xi)(2+\xi) & 144 \xi^{4} & -144 \xi^{4} & 36 \xi^{3}(1+\xi)^{2} \\
-36 \xi^{3}(1+\xi) & -144 \xi^{4} & 144 \xi^{4} & 36 \xi^{3}(1+\xi)^{2} \\
-9(1-\xi) \xi^{2}(1+\xi)^{2} & 36 \xi^{3}(1+\xi)^{2} & 36 \xi^{3}(1+\xi)^{2} & -9 \xi^{2}\left(1-\xi^{2}\right)^{2}
\end{array}\right)
$$

\section{B.3 BH amplitude squared}

cI

$$
C_{0, \mathrm{unp}}^{\mathrm{BH}}=\frac{8\left(2-2 y+y^{2}\right)}{3(1+\xi)^{2}}\left(2 \xi^{2} G_{2}^{2}+\left(1-\frac{\Delta_{\mathrm{min}}^{2}}{\Delta^{2}}\right)\left(1-\xi^{2}\right)\left(3 G_{1}^{2}-4 \tau G_{1} G_{3}+4 \tau^{2} G_{3}^{2}\right)\right)
$$

$$
\begin{aligned}
& C_{0, \mathrm{LP}}^{\mathrm{BH}}=\frac{-8(2-y) y \lambda \xi}{(1+\xi)^{3}} G_{2}\left(\xi(1+2 \xi) G_{2}+2\left(1-\frac{\Delta_{\min }^{2}}{\Delta^{2}}\right)\left(1-\xi^{2}\right)\left(G_{1}-\tau G_{3}\right)\right)
\end{aligned}
$$

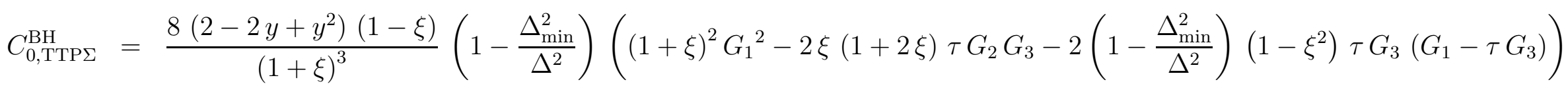




\section{Estimate of target mass corrections}

Target mass corrections for a scalar and spin-1/2 target have been evaluated in Ref. [37, where $\Delta^{2} / \mathcal{Q}^{2}$ corrections have been neglected. This result will serve us to derive the scaling law of the target mass corrections with respect to the atomic mass number $A$ for a general Compton process in the light-cone dominated region. It is sufficient to consider the simplest case of a scalar target. Then the target mass corrections in the parity even sector read for the Compton amplitude

$$
\begin{gathered}
\mathcal{F}_{1}(\xi, \eta)=\int_{\Omega} d y d z h(y, z)\left[C_{1}^{(0)}\left(\frac{y+\eta z}{\xi}-i 0\right)+\frac{\xi^{2} M^{2} y^{2}}{Q^{2}(y+\eta z)^{2}} C_{1}^{(1)}\left(\frac{y+\eta z}{\xi}-i 0\right)\right. \\
\left.+\mathcal{O}\left(\frac{\xi^{4} M^{4}}{Q^{4}}\right)+\left\{\begin{array}{l}
y \rightarrow-y \\
z \rightarrow-z
\end{array}\right\}\right] .
\end{gathered}
$$

An analogous formula for the other leading twist amplitude $\mathcal{F}_{2}$ holds true. Here $h(y, z)$ denotes the DD and the coefficients

$$
C_{1}^{(0)}\left(\Xi^{-1}\right)=-\frac{1}{1+\Xi}, \quad \text { and } \quad C_{1}^{(1)}\left(\Xi^{-1}\right)=\frac{1}{(1+\Xi)^{2}}+2 \ln \left(\frac{\Xi}{1+\Xi}\right),
$$

depend on the variable $\Xi=\xi /(y+\eta z)$. Let us set the mass $M$ and the scaling variables in Eq. (165) equal to the nucleon ones: $M_{N}, \xi_{N}, \eta_{N}$. The Compton amplitude for a nucleus A at smaller values of the Bjorken variable ${ }^{14}$ follows from the replacements:

$$
M_{N} \rightarrow M=A M_{N}, \xi_{N} \rightarrow \xi \sim \xi_{N} / A, \eta_{N} \rightarrow \eta \sim \eta_{N} / A, \text { and } h \rightarrow h^{A} .
$$

Corresponding to our nuclei GPD model (70) and the GPD definition (50) in terms of a DD, we read off the scaling law for the nucleus DD:

$$
h^{A}(y, z) \propto \theta(|A y \pm z| \leq 1) h(A y, z) .
$$

Changing the integration variable $y=y^{\prime} / A$ and renaming $y^{\prime} \rightarrow y$, we find that the Compton amplitude for a nucleus target reads

$$
\begin{aligned}
F_{1}^{A}(\xi, \eta) \propto \int_{\Omega} d y d z h(y, z)\left[C_{1}^{(0)}\left(\frac{y+\eta_{N} z}{\xi_{N}}-i 0\right)+\frac{\xi_{N}^{2} M_{N}^{2} y^{2}}{Q^{2}\left(y+\eta_{N} z\right)^{2}} C_{1}^{(1)}\left(\frac{y+\eta_{N} z}{\xi_{N}}-i 0\right)\right. \\
\left.+\mathcal{O}\left(\frac{\xi_{N}^{4} M_{N}^{4}}{Q^{4}}\right)+\left\{\begin{array}{l}
y \rightarrow-y \\
z \rightarrow-z
\end{array}\right\}\right] .
\end{aligned}
$$

As we realize by comparison with Eq. (165), setting $M=M_{N}, \xi=\xi_{N}$, and $\eta=\eta_{N}$, the target mass corrections do not scale with $A$ in respect to the leading twist-two term. It can be shown in the same way from Eqs. (30) and (31) in Ref. 37] that also the resummed target mass corrections possess the same property. From the same representation it also follows that this statement is true for a target with non-zero spin content, including the parity odd sector.

Beside this kind of corrections also dynamical ones, which are expressed in terms of multiparton correlation functions, appear in the CFFs. Unfortunately, only little is known about them and it remains an open task to consider the full twist-four sector.

\footnotetext{
${ }^{14}$ We only use this condition to simplify the discussion. For larger values of $x_{\mathrm{B}}$ one should take the relation $\xi=(1+\xi) \xi_{N} /\left(1+\xi_{N}\right) A$.
} 


\section{References}

[1] D. Müller, D. Robaschik, B. Geyer, F.-M. Dittes, and J. Hořejši, Fortschr. Phys. 42, 101 (1994), hep-ph/9812448.

[2] A. Radyushkin, Phys. Lett. B380, 417 (1996), hep-ph/9604317.

[3] X. Ji, Phys. Rev. D55, 7114 (1997), hep-ph/9609381.

[4] M. Diehl, T. Gousset, B. Pire, and J. P. Ralston, Phys. Lett. B411, 193 (1997), hep-ph/9706344.

[5] A. Belitsky, D. Müller, L. Niedermeier, and A. Schäfer, Nucl. Phys. B593, 289 (2001), hep-ph/0004059.

[6] A. Belitsky, D.Müller, A. Kirchner, and A.Schäfer, Phys. Rev. D64, 116002 (2001), hep-ph/0011314.

[7] A. V. Belitsky, D. Müller, and A. Kirchner, Nucl. Phys. B629, 323 (2002), hep-ph/0112108.

[8] E. R. Berger, M. Diehl, and B. Pire, Eur. Phys. J. C23, 675 (2001), hep-ph/0110062.

[9] M. Guidal and M. Vanderhaeghen, Phys. Rev. Lett. 90, 012001 (2003), hep-ph/0208275.

[10] A. V. Belitsky and D. Müller, Phys. Rev. Lett. 90, 022001 (2003), hep-ph/0210313.

[11] J. Collins and A. Freund, Phys. Rev. D59, 074009 (1999), hep-ph/9801262.

[12] X. Ji and J. Osborne, Phys. Rev. D58, 094018 (1998), hep-ph/9801260.

[13] X. Ji, Phys. Rev. Lett. 78, 610 (1997), hep-ph/9603249.

[14] M. Burkardt, Phys. Rev. D62, 071503 (2000), hep-ph/0010082.

[15] J. Ralston and B. Pire, Phys. Rev. D66, 111501 (2002), hep-ph/0110075.

[16] M. Diehl, Eur. Phys. J. C25, 223 (2002), hep-ph/0205208.

[17] A. V. Belitsky and D. Müller, Nucl. Phys. A711, 118 (2002), hep-ph/0206306.

[18] J. Collins, L. Frankfurt, and M. Strikman, Phys. Rev. D56, 2982 (1997), hep-ph/9611433.

[19] C. Adloff et al. (H1 Coll.), Phys. Lett. B517, 47 (2001), hep-ex/0107005.

[20] P.R.S. Saull (ZEUS Coll.), Prompt photon production and observation of deeply virtual compton scattering, hep-ex/0003030, 2000.

[21] A. Airapetian et al. (HERMES Coll.), Phys. Rev. Lett. 87, 182001 (2001), hep-ex/0106068.

[22] S. Stepanyan et al. (CLASS Coll.), Phys. Rev. Lett. 87, 182002 (2001), hep-ex/0107043.

[23] HERMES, F. Ellinghaus, Nucl. Phys. A711, 171 (2002), hep-ex/0207029. 
[24] P. Pobylitsa, Virtual Compton Scattering in the generalized Bjorken region and positivity bounds on generalized parton distributions, hep-ph/0211160, 2002.

[25] B. Pire, J. Soffer, and O. Teryaev, Eur. Phys. J. C8, 103 (1999), hep-ph/9804284.

[26] A. Radyushkin, Phys. Rev. D59, 014030 (1999), hep-ph/9805342.

[27] HERMES, F. Ellinghaus, R. Shanidze, and J. Volmer, (2002), hep-ex/0212019.

[28] P. Hoodbhoy, R. Jaffe, and A. Manohar, Nucl. Phys. B312, 571 (1989).

[29] S. Brodsky, C.-R. Ji, and G. Lepage, Phys. Rev. Lett. 51, 83 (1983).

[30] R. Gilman and F. Gross, J. Phys. G28, R37 (2002), nucl-th/0111015.

[31] M. Garçon and J. V. Orden, Adv.Nucl.Phys. 26, 293 (2001), nucl-th/0102049.

[32] E. R. Berger, F. Cano, M. Diehl, and B. Pire, Phys. Rev. Lett. 87, 142302 (2001), hep-ph/0106192.

[33] A. Kirchner and D. Müller, Predictions for deeply virtual compton scattering on a spin-one target, hep-ph/0202279, 2002.

[34] F. Cano and B. Pire, Nucl. Phys. A711, 133 (2002), hep-ph/0206215.

[35] F. Cano and B. Pire, Deeply Virtual Compton Scattering on Spin-1 Nuclei, hep-ph/0211444, 2002.

[36] M. Polyakov, Generalized parton distributions and strong forces inside nucleons and nuclei, hep-ph/0210165, 2002.

[37] A. Belitsky and D. Müller, Phys. Lett. B507, 173 (2001), hep-ph/0102224.

[38] A. Belitsky and D. Müller, Nucl. Phys. B 589, 611 (2000), hep-ph/0007031.

[39] P. Hoodbhoy and X. Ji, Phys. Rev. D58, 054006 (1998), hep-ph/9801369.

[40] A. Belitsky and D. Müller, Phys. Lett. B486, 369 (2000), hep-ph/0005028.

[41] M. Diehl, Eur. Phys. J. C19, 485 (2001), hep-ph/0101335.

[42] N. Kivel and L. Mankiewicz, Eur. Phys. J. C21, 621 (2001), hep-ph/0106329.

[43] G. Passarino, Nucl. Phys. B 237, 249 (1984).

[44] I. Anikin, B. Pire, and O. Teryaev, Phys. Rev. D 62, 071501 (2000), hep-ph/0003203.

[45] A. V. Radyushkin and C. Weiss, Phys. Rev. D63, 114012 (2001), hep-ph/0010296.

[46] A. D. Martin, W. J. Stirling, and R. G. Roberts, Phys. Lett. B354, 155 (1995), hep-ph/9502336. 
[47] S. Stein et al., Phys. Rev. D12, 1884 (1975).

[48] M. V. Polyakov and C. Weiss, Phys. Rev. D60, 114017 (1999), hep-ph/9902451.

[49] V. Petrov et al., Phys. Rev. D57, 4325 (1998), hep-ph/9710270.

[50] P. Schweitzer, S. Boffi, and M. Radici, Phys. Rev. D66, 114004 (2002), hep-ph/0207230.

[51] K. Goeke, M. Polyakov, and M. Vanderhaeghen, Prog. Part. Nucl. Phys. 47, 401 (2001), hep-ph/0106012.

[52] A. Freund and M. F. McDermott, Phys. Rev. D65, 091901 (2002), hep-ph/0106124 Phys. Rev. D65, 074008 (2002), hep-ph/0106319; Eur. Phys. J. C23, 651 (2002), hep-ph/0111472.

[53] A. Belitsky, A. Kirchner, D. Müller, and A. Schäfer, Phys.Lett. B510, 117 (2001), hep-ph/0103343.

[54] A. P. Kobushkin and A. I. Syamtomov, Phys. Atom. Nucl. 58, 1477 (1995), hep-ph/9409411.

[55] D. Abbott et al. (JLAB T20 Coll. ), Eur. Phys. J. A7, 421 (2000), nucl-ex/0002003.

[56] F. E. Close and S. Kumano, Phys. Rev. D42, 2377 (1990).

[57] M. Contalbrigo, A First Measurement of the Tensor-Polarized Structure Function $\mathrm{b}_{1}^{\mathrm{d}}$, hep-ex/0211014, 2002.

[58] J. Cooke and G. Miller, Phys. Rev. C66, 034002 (2002), nucl-th/0112037.

[59] T. P. Cheng and W.-K. Tung, Phys. Rev. Lett. 24, 851 (1970).

[60] S. Brodsky, F. Close, and J. Gunion, Phys. Rev. D5, 1384 (1972).

[61] T. Gehrmann and W. J. Stirling, Phys. Rev. D53, 6100 (1996), hep-ph/9512406.

[62] V. Guzey and M. Strikman, DVCS on spinless nuclear targets in impulse approximation, hep-ph/0301216, 2003.

[63] S. Brodsky and J. Hiller, Phys. Rev. D46, 2141 (1992). 